\title{
Estimadores de parâmetro consistentes para modelos de grafo aleatório e estudo sobre a relação entre a rede modo padrão do cérebro e o volume do corpo caloso
}

Suzana de Siqueira Santos

\author{
TESE APRESENTADA AO \\ INSTituTo DE MATEMÁTiCA E EsTATÍSTICA \\ DA Universidade de SÃo PaUlo \\ PARA OBTENÇÃO DO TÍTULO DE \\ DOUTORA EM CIÊNCIAS
}

Programa: Ciência da Computação

Orientador: Prof. Dr. André Fujita

Durante o desenvolvimento deste trabalho a autora recebeu auxílio financeiro da CAPES e da FAPESP (Processos 2015/21162-4 e 2017/12074-0)

São Paulo

28 de fevereiro de 2020 



\title{
Estimadores de parâmetro consistentes para modelos de grafo aleatório e estudo sobre a relação entre a rede modo padrão do cérebro e o volume do corpo caloso
}

\author{
Suzana de Siqueira Santos
}

Esta versão da tese contém as correções e alterações sugeridas pela Comissão Julgadora durante a defesa da versão original do trabalho, realizada em 28 de fevereiro de 2020.

Uma cópia da versão original está disponível no Instituto de Matemática e Estatística da Universidade de São Paulo.

Comissão Julgadora:

- Prof. Dr. André Fujita (orientador) - IME-USP

- Prof $^{a}$. Dr ${ }^{\mathrm{a}}$. Joana Bisol Balardin - Albert Einstein

- Prof. Dr. Fabricio Martins Lopes - UTFPR

- Prof ${ }^{a}$. Dra ${ }^{a}$. Florência Graciela Leonardi - IME-USP

- Prof. Dr. Francisco Aparecido Rodrigues - ICMC-USP 
Autorizo a reprodução e divulgação total ou parcial deste trabalho, por qualquer meio convencional ou eletrônico, para fins de estudo e pesquisa, desde que citada a fonte. 
Dedico este trabalho a Fuliana, Gabi, Cris, Samuel e Lady pelas horas que lhes foram roubadas.

Dedico-o também às memórias de Antonio, Antonia, José, Alberto e Maria, alicerces da minha vida. 



\section{Agradecimentos}

Muitas pessoas queridas contribuíram para a conclusão deste trabalho. Arrisco-me aqui a enumerar algumas, embora já saiba que não serei capaz de enumerar todas.

Destaco a contribuição do Prof. André Fujita, que orientou o desenvolvimento desta tese com admirável empenho e que me ensina desde a iniciação científica a fazer ciência. Seus conselhos, ideias, as oportunidades oferecidas e disponibilidade foram fundamentais para meu desenvolvimento como pesquisadora e para a evolução deste trabalho. Dedico meus sinceros agredecimentos à Profa. Catherine Matias por ter me recebido tão bem em seu laboratório, pelos incentivos e contribuição aos resultados desta tese e por me inspirar de tantas formas.

Agradeço também a todos os professores e colegas que contribuíram enormemente para esta tese com suas dicas e opiniões. Em especial, agradeço aos Professores Anatoli Iambartsev pelas dicas e sugestões dadas no exame de qualificação. Aos Professores Joana Balardin, Francisco Rodrigues e Florência Leonardi pelas melhorias sugeridas para a versão corrigida da tese e ao professor Fabrício Lopes pelas valiosas contribuições tanto no exame de qualificação quanto na defesa da tese. Ao Prof. Alexandre Patriota agradeço por me ajudar incansável e pacientemente a adquirir conhecimentos teóricos totalmente novos para mim, ao Prof. João Ricardo Sato e aos colegas Grover e Maciel pelo constante suporte para trabalhar com dados neurociência. Em particular, agradeço a Taiane, pela ajuda nos estudos, conselhos, conversas e companheirismo ao longo do doutorado.

Agradeço à FAPESP e à CAPES por terem fornecido as condições financeiras para a realização deste trabalho e à USP e ao IME por oferecer toda a estrutura para o desenvolvimento do trabalho e pelo ambiente enriquecedor.

Agradeço à Katia, secretária da $\mathrm{CCP}$, ao coordenador do programa Roberto Hirata e à Ana da CPG por toda a ajuda para a realização do meu doutorado. Também sou muito grata aos excelentes professores do Departamento de Computação do IME-USP e do departamento de Estatística, que tanto me inspiraram e ensinaram em suas aulas. Agradeço à Ana Carla, Edina e Cristiane, secretárias do DCC-IME-USP pela dedicação e 
ao Geraldo, pela amizade e ajuda no dia-a-dia do IME.

Agradeço aos membros do GEEG, Eduardo Lira, Waldir, Vinícius, Eduardo Laurentino e demais membros já citados pelo companheirismo nos estudos, momentos de diversão, risadas e discussões valiosas sobre álgebra linear, teoria da informação, grafos e estatística. Não poderia deixar de mencionar também demais amigos e colegas de laboratório (e agregados do LIAMF), que fizeram o meu doutarado mais feliz, a Lucy, o Marcos, a Julissa, o Juan, a Sabrina, a Ana, o Antorio, o Eric, a Irina, o Mateus, o Caio Francisco, Caio Braz, a Erika, o Tiago e a Camila.

Dedico também um agradecimento sincero aos meus colegas do LPSM em Paris, que tornaram meu intercâmbio mais que especial e divertido: o Henri Elad-Altman, Paul Melotti, Carlo, Karen, Isao, Alexandra, Vivien, Eric Saias, Nicolas, Rancy, Thibaut, Clément, Lucas, Léa, Guillermo. Em particular, gostaria de agradecer à Flaminia Zane por ter se tornado uma amiga infalível, me fortalecendo e estando sempre ao meu lado.

Destaco também o papel tão importante que a comissão de recepção de pós-graduação (RPG) do IME teve na minha formação como pessoa e profissional. Agradeço pela dedicação inspiradora e conversas edificantes aos meus queridos amigos Fernando, Bartira, Wil, Rafael e Alexandre.

Agradeço também aos meus colegas Ana Luiza e Kaique pela oportunidade em participar ainda que brevemente da organização dos seminários Café, Giz e Demonstração, que me introduziram a novos colegas e conhecimentos.

Agradeço aos amigos da equipe Fantinhas, DJ, José, Gustavo, André e Diego, pelo trabalho em durante o SPSAS e por tantos momentos divertidos e conselhos recebidos.

Destaco também a presença de meus amigos desde a graduação, pelos momentos de alegria e descontração: a Mônica, Susanna, o Igor, o Gustavo, a Nádia, o Goroba, o Felipe, o Manzo, o Diego, o Jef, a Jéssica, o Katague e tantos outros! Agradeço especialmente ao Jackson pela companhia infalível, conselhos e discussões enriquecedoras.

Não poderia deixar de mencionar minhas amigas desde a infância: a Nébs, a Keyla, a Ju, a Amanda, a Loly e a Livia.

Agradeço aos membros do Tecs USP pelo ativismo social e pela oportunidade de participar de projetos inspiradores de educação e às queridas Deise, Rosana, Claudia, Grazi, Malu, Nathalie, Natasha e Gisele pelo companheirismo no trabalho com os animais e auxílio nos meus períodos de ausência.

Dedico um agradecimento especial ao Samuel pelas contribuições a este trabalho e por seu amor e companhia. Seus conselhos e todas as formas de apoio trouxeram forças para a 
conclusão do doutorado. Agradeço à sua família, Luiza, Depa e Jacque por toda a torcida e carinho.

Por fim, agradeço à minha família, a quem devo grande parte do que aprendi e das oportunidades que tive para chegar até aqui. Agradeço à minha mãe Juliana e ao meu pai Antonio por tudo que fizeram por mim. Às minhas irmãs e grandes amigas Ana Gabriela e Cristiana e aos meus cunhados Michel e Wallace, agradeço pelos exemplos, apoio e por tantos momentos divertidos. À minha cachorrinha Lady por me fazer uma pessoa melhor e mais feliz. Aos meus avós Antonia, José, Alberto e Maria, tios Inês, Chico e Lolia, primos e primas, agradeço pelo carinho e incentivos.

A você, leitor(a), agradeço pela paciência e potencial interesse neste trabalho. 



\section{Resumo}

Suzana de Siqueira Santos. Estimadores de parâmetro consistentes para modelos de grafo aleatório e estudo sobre a relação entre a rede modo padrão do cérebro e o volume do corpo caloso. Tese (Doutorado). Instituto de Matemática e Estatística, Universidade de São Paulo, São Paulo, 2020.

Grafos possibilitam estudar o funcionamento de diversos sistemas, como redes biológicas e sociais. Nesse contexto, surge o problema (i) de selecionar um modelo de grafo aleatório e um conjunto de parâmetros que melhor se ajustem a uma rede do mundo real, buscando interpretar e predizer seu comportamento. Dada uma sequência de redes e valores observados, temos adicionalmente o problema (ii) de correlacioná-los. Para (i), Takahashi e colegas propuseram um método baseado nas densidades dos espectrais (distribuição dos autovalores da matriz de adjacência) cuja principal vantagem é a generalidade. Nós propusemos adaptações, baseadas na norma $\ell_{1}$ entre densidades espectrais e entre distribuições acumuladas, que nos levaram à derivação de resultados teóricos sobre a consistência do estimador de parâmetro. Finalmente, o problema (ii) é abordado no Transtorno do Espectro Autista (TEA), cujas sub-classificações em Asperger e autismo têm bases neurais pouco conhecidas. Como há evidências de alterações da rede modo padrão em TEA, comparamos a relação dessa rede com a maior estrutura de matéria branca do cérebro (corpo caloso) entre Asperger e autismo. Nossos resultados sugerem que essa relação é maior em Asperger do que em autismo na região anterior do corpo caloso e que o maior autovalor do grafo é capaz de capturar a relação com o parâmetro estimado.

Palavras-chave: Estimador de parâmetro. Estimador de mínimo contraste. Grafo aleatório. Densidade espectral. Maior autovalor. Autismo. Asperger. Corpo caloso. Conectividade funcional. Rede modo padrão do cérebro. 



\begin{abstract}
Suzana de Siqueira Santos. Consistent parmeter estimators for random graph models and study of the relation between the default mode network and the corpus callosum volume. Thesis (Doctorate). Institute of Mathematics and Statistics, University of São Paulo, São Paulo, 2020.
\end{abstract}

Graphs are used to study the behavior of several systems, such as social and biological networks. In this context, a common problem is (i) selecting the random graph model and set of parameters that best fit the real world network and interpreting/predicting its behavior. Given a sequence of networks and observed values, we have additionally the problem (ii) of studying their interaction. For (i), Takahashi and colleagues proposed a method based on the spectral density (distribuition of the eigenvalues of the adjacency matrix) whose main advantage is its generality: it works for different random graph models. We proposed adaptations based on the $\ell_{1}$ norm between spectral densities and between cumulative distributions of the eigenvalues, which led us to the derivation of theoretical results on the consistency of the parameter estimators. Finally, we study problem (ii) in the context of the Autism Spectrum Disorder (ASD), whose sub-groups Asperger and autism have little known neural bases. As there are evidences of alterations of the default mode network in ASD, we compared the relation between this network and the largest white matter structure of the brain (corpus callosum) between Asperger and autism. Our results suggest that this relation is greater in Asperger than in autism in the anterior region of the corpus callosum and that the largest eigenvalue can capture the relation with the estimated random graph parameter.

Keywords: Parameter estimator. Minimum contrast estimator. Random graph. Spectral density. Largest eigenvalue. Autism. Asperger. Corpus callosum. Functional connectivity. Default-mode network of the brain. 



\title{
Lista de Abreviaturas
}

\author{
ABIDE Autism Brain Imaging Data Exchange \\ BA Modelo de Barabási-Albert \\ BE Modelo bloco estocástico \\ CC Corpo caloso \\ $\mathrm{CIH}$ Conectividade inter-hemisférica \\ DAA Distribuição acumulada dos autovalores \\ DEE Densidade espectral empírica \\ DR Modelo $d$-regular \\ DSM Diagnostic and Statistical Manual of Mental Disorders \\ ER Modelo de Erdős-Rényi \\ FD Framewise Fisplacement (deslocamento de quadros) \\ GE Modelo geométrico \\ KL Divergência de Kullback-Leibler \\ fMRI Imagem por ressonância magnética funcional \\ sMRI Imagem por ressonância magnética estrutural \\ TEA Transtorno do espectro autista \\ WS Modelo de Watts-Strogatz
}




\section{Lista de Figuras}

3.1 Grafo de tamanho 500 gerado pelo modelo Erdős-Rényi e sua densidade espectral empírica. . . . . . . . . . . . . . . . . . . . . 14

3.2 Densidade espectral empírica e teórica do modelo bloco estocástico. . . .

3.3 Grafo de tamanho 500 gerado pelo modelo $d$-regular e sua densidade espectral empírica. . . . . . . . . . . . . . . . . . . . . . . . . . 19

3.4 Grafo de tamanho 500 gerado pelo modelo geométrico e sua densidade espectral empírica. . . . . . . . . . . . . . . . . . . . .

3.5 Grafo de tamanho 500 gerado pelo modelo Watts-Strogatz e a densidade espectral empírica. . . . . . . . . . . . . . . . . .

3.6 Grafo de tamanho 500 gerado pelo modelo Barabási-Albert e a densidade espectral empírica. . . . . . . . . . . . . . . . . . 22

4.1 Entropia espectral do grafo. . . . . . . . . . . . . . . 27

5.1 Distribuição do estimador de parâmetro baseado na divergência de Kullback-Leibler entre densidades espectrais estimadas pelo critério de Silverman. . . . . . . . . . . . . . . . . . . . .

5.2 Boxplots dos estimadores de parâmetro para grafos BA de tamanho $n=500$ com expoente de escala $p_{s}=1.4 \ldots \ldots \ldots \ldots \ldots$

5.3 Boxplots dos estimadores de parâmetro para grafos BA de tamanho $n=100$ com expoente de escala $p_{s}=1.1,1.3,1.5,1.7,1.9 . \ldots \ldots \ldots$

5.4 Boxplots dos estimadores de parâmetro para grafos BA de tamanho $n=500$ com expoente de escala $p_{s}=1.1,1.3,1.5,1.7,1.9 . \ldots 37$

5.5 Boxplots dos estimadores de parâmetro para grafos ER, DR, GE e WS. . . 39

5.6 Bagplots dos estimadores de parâmetro para grafos BE. . . . . . . . . . . 40

5.7 Parâmetro ajustado médio para o modelo ER. . . . . . . . . . . . . . . . 45

5.8 Parâmetro ajustado médio para o modelo DR. . . . . . . . . . . . . 46

5.9 Parâmetro ajustado médio para o modelo GE. . . . . . . . . . . . . . . . . 47

5.10 Parâmetro ajustado médio para o modelo WS. . . . . . . . . . . . . . . . 48 
5.11 Parâmetro ajustado médio para o modelo BA. . . . . . . . . . . . . . . 49

5.12 Distribuição do estimador de parâmetro para os modelos ER e DR. . . . . 51

5.13 Distribuição do estimador de parâmetro para os modelos GE, WS e BA . 52

7.1 Segmentação do corpo caloso em 5 sub-regiões: posterior, posterior médio, central, anterior médio e anterior. . . . . . . . . . . . 63

7.2 Silhueta dos agrupamentos da rede de funcional do cérebro. . . . . . . . 67

7.3 Mapeamento dos módulos funcionais do cérebro. . . . . . . . . . . . 68

7.4 Gráficos de dispersão entre o volume da rergião anterior do corpo caloso e medidas de conectividade funcional. . . . . . . . . . . . . . 71

7.5 Medidas de conectividade funcional e de volumes cerebrais médias. . . . 72

\section{Lista de Tabelas}

3.1 Convergência da densidade espectral empírica em diferentes modelos.

5.1 Valores médios e desvio padrão obtidos pelo procedimento de Takahashi e colegas para estimação de parâmetros. . . . . . . . . . . . . .

6.1 Seleção de modelos baseada na norma $\ell_{1}$ para grafos gerados $\operatorname{com} \beta=0.1 . \quad 56$

6.2 Seleção de modelos baseada na norma $\ell_{1}$ para grafos gerados $\operatorname{com} \beta=0.5$. 57

6.3 Seleção de modelos baseada na norma $\ell_{1}$ para grafos gerados $\operatorname{com} \beta=0.9 . \quad 58$

7.1 Resumo de informações demográficas dos dados do ABIDE. . . . . . . . . 64

7.2 Comparação da associação entre a rede modo padrão e o volume de subregiões do corpo caloso em autismo e Asperger. . . . . . . . . . . . .

A.1 Coeficientes estimados no modelo linear entre maior autovalor da rede modo padrão e volume do corpo caloso anterior para indivíduos com autismo. 79

A.2 Coeficientes estimados no modelo linear entre maior autovalor da rede modo padrão e volume do corpo caloso anterior para indivíduos com Asperger. . . . . . . . . . . . . . . . . .

A.3 Coeficientes estimados no modelo linear entre maior autovalor da rede modo padrão e volume do corpo caloso anterior para controles. . . . . . 
B.1 Comparação da associação entre a rede modo padrão e o volume de subregiões do corpo caloso entre indivíduos com autismo e indivíduos com síndrome de Asperger a partir de dados de ressonância magnética funcional do cérebro sem aplicação do procedimento de scrubbing. . . . . . . . . .

B.2 Coeficientes e p-valores dos modelos lineares ajustados para o maior autovalor em controles, indivíduos com autismo e Asperger a partir de dados sem aplicação do procedimento de scrubbing. . . . . . . . . . . . . . . 


\section{Sumário}

1 Introdução 1

1.1 Objetivos ........................ 4

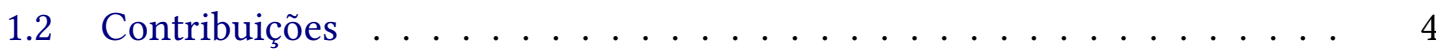

1.2.1 Produção de textos técnicos e científicos . . . . . . . . . . 4

1.2 .2 Software . . . . . . . . . . . . . . . . . . 5

2 Notação e definições $\quad 7$

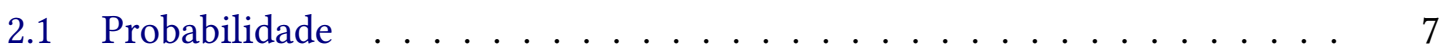

2.1.1 Definições . . . . . . . . . . . . . . . . . 7

2.1.2 Teorema de convergência dominada . . . . . . . . . . . . . 10

2.2 Grafos . . . . . . . . . . . . . . . . . . . 10

2.3 Grafos aleatórios . . . . . . . . . . . . . . . . . . . 11

2.4 Notação assintótica . . . . . . . . . . . . . . . . . . . . . . . . 12

3 Densidade espectral de grafos aleatórios 13

3.1 Lei semi-circular de Wigner . . . . . . . . . . . . . . . . . . . 13

3.2 Erdős-Rényi (ER) f . . . . . . . . . . . . . . . . . . . 13

3.3 Modelo bloco estocástico $(\mathrm{BE}) \quad \ldots \ldots \ldots \ldots$

3.4 Grafo aleatório $d$-regular $(\mathrm{DR}) \ldots \ldots \ldots \ldots \ldots$

3.5 Modelo geométrico $(\mathrm{GE}) \ldots \ldots \ldots$

3.6 Modelo de Watts-Strogatz (WS) . . . . . . . . . . . . . . . . . . 20

3.7 Modelo de configuração . . . . . . . . . . . . . . . . . . . . . . . . 21

3.8 Modelo de ligação preferencial . . . . . . . . . . . . . . . . . . . . 22

3.9 Conclusões . . . . . . . . . . . . . . . . . . . . . . . 23

4 Teoria da Informação para grafos 25

4.1 Entropia espectral . . . . . . . . . . . . . . . . . 25

4.2 Divergência de Kullback-Leibler . . . . . . . . . . . . . . . . . . . 28 
5 Estimadores de parâmetro baseados na distribuição dos autovalores $\quad 29$

5.1 Descrição do método . . . . . . . . . . . . . . . . . 30

5.2 Método original . . . . . . . . . . . . . . . . 32

5.3 Adaptações . . . . . . . . . . . . . . . . . . . 33

5.4 Propriedades de convergência . . . . . . . . . . . . . . . 41

5.4.1 Estimadores de mínimo contraste . . . . . . . . . . . . . 41

5.4.2 Estimadores baseados na norma $\ell_{1}$ entre distribuições de autovalores 42

5.4 .3 Simulações . . . . . . . . . . . . . . . . . . . . 44 44

5.5 Distribuição do estimador de parâmetro . . . . . . . . . . . . . . . . 50

5.6 Discussões . . . . . . . . . . . . . . . . . . 53

6 Seleção de modelos de grafo aleatório 55

7 Relação entre a rede funcional do cérebro e o volume do corpo caloso em Asperger e autismo $\quad 59$

7.1 Asperger e autismo . . . . . . . . . . . . . . . . . . 59

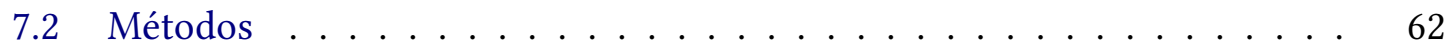

7.2.1 Conjunto de dados ABIDE . . . . . . . . . . . . . 62

7.2.2 Rede modo padrão . . . . . . . . . . . . . . . . 64

7.2.3 Relação entre a rede modo padrão e o corpo caloso . . . . . . . . 65

7.2.4 Seleção de modelos e estimação de parâmetros . . . . . . . . . . 66

7.2 .5 Comparação entre os grupos . . . . . . . . . . . . . . 66

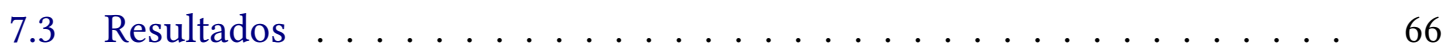

7.4 Discussão . . . . . . . . . . . . . . . . . . . . 73

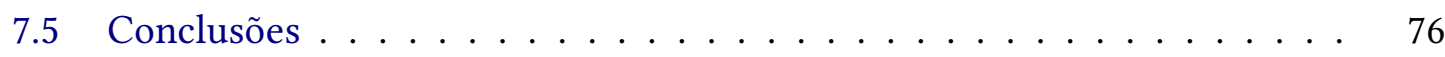

8 Considerações finais $\quad 77$

\section{Apêndices}

A Coeficientes estimados nos controles, indivíduos com autismo e Asperger 79

B Resultados obtidos antes do procedimento de scrubbing 83 


\section{Capítulo 1}

\section{Introdução}

Grafos (ou redes) são estruturas matemáticas fundamentais para modelar sistemas reais em que os componentes interagem entre si. Podemos citar como exemplos as redes sociais (Facebook, ResearchGate, etc), redes de regulação genética (DAVIDSON e LEVIN, 2005), redes de interação proteína-proteína (PELLEGRINI et al., 2004) e redes de coativação entre regiões do cérebro (Heuvel e Hulshoff Pol, 2010). Usualmente as redes do mundo real apresentam flutuações, como, por exemplo, em Biologia, em que há heterogeneidade entre indivíduos sob uma mesma condição e variabilidade ao longo do tempo nos processos dinâmicos. Assim representar uma rede do mundo real como um grafo determinístico pode não ser apropriado.

Para testar se duas redes do mundo real são diferentes, abordagens tradicionais verificam se os grafos são exatamente iguais (quando o conjunto de vértices é o mesmo), se compartilham certas propriedades estruturais ou se são isomórficos (MuELLER et al., 2013). Mas, para muitas aplicações, deseja-se saber se a diferença entre os grafos é maior do que se esperaria pela variabilidade da população ou flutuações estocásticas. Nesse contexto, é mais adequado assumir que as redes observadas são realizações de processos aleatórios.

Um dos primeiros modelos de grafo aleatório descritos na literatura é o modelo de ERDős e RÉNYI, 1959, no qual, dados $n$ vértices rotulados, as arestas são inseridas aleatoriamente de forma independente com uma probabilidade $p$. Como muitas redes do mundo real não compartilham as características dos grafos de Erdős-Rényi, outros modelos foram propostos para melhor explicar os sistemas do mundo real. Exemplos incluem a família de modelos de grafos aleatórios exponenciais (Frank e STRAuss, 1986), o modelo de bloco estocástico (Fienberg e Wasserman, 1981; Holland et al., 1983; Snijders e Nowicki, 1997) (que pode ser interpretado como uma mistura de grafos de Erdős-Rényi), o modelo geométrico (Penrose, 2003) (em que existe uma relação espacial entre os vértices conectados), o modelo de Watts-Strogatz (Watts e Strogatz, 1998) (grafos com grupos de vértices densamente conectados), modelo de Barabási-Albert (BARABÁsi e AlBERT, 1999) (grafos cuja distribuição dos graus segue a lei de potência).

Ao assumir que uma rede do mundo real foi gerada por um determinado modelo de grafo aleatório, o próximo passo é estimar o parâmetro que maximiza a probabilidade de 
ocorrência do grafo observado. Há estimadores de parâmetro de máxima verossimilhança para alguns modelos de grafo aleatório, como Erdős-Rényi, bloco estocástico (SNIJDERS e Nowicki, 1997; Allman et al., 2011; Ambroise e Matias, 2012) e a família de grafos aleatórios exponenciais (Geyer e Thompson, 1992; CAImo e Friel, 2011). Contudo, para outros modelos, como Watts-Strogatz e Barábasi-Albert, não se conhece um bom estimador.

Assim, TAKAHASHi et al., 2012 propuseram um procedimento aplicável a diversos modelos para estimar parâmetros a partir da minimização na divergência de KullbackLeibler (KL) entre densidades espectrais (distribuição do conjunto de autovalores de matriz de adjacência). Além disso, o método fornece uma medida de ajuste do modelo, que pode ser aplicada para selecionar o modelo que mais se aproxima do grafo observado a partir de uma lista pré-definida.

A escolha da densidade espectral é baseada no fato de o espectro (conjunto dos autovalores da matriz de adjacência) descrever diversas propriedades estruturais dos grafos, como o diâmetro, número de passeios e cliques (VAn Mieghem, 2010; TAKahashi et al., 2012). Além disso, a densidade espectral pode ser utilizada para caracterizar famílias de grafos aleatórios, como sugerido em experimentos de simulação (FARKAs et al., 2001), e demonstrado teoricamente para o modelo Erdős-Rényi (TrAN et al., 2013), bloco estocástico (Avrachenkov et al., 2015), d-regular (McKay, 1981; Dumitriu e PAL, 2012; Tran et al., 2013) e geométrico (BlACKWEll et al., 2007; HAMidouche et al., 2019).

O uso da densidade espectral também se mostrou efetivo para testar se grafos foram gerados pelo mesmo processo probabilístico. TAKAHAshi et al., 2012 e Fujita, M. C. VIdAL et al., 2017 desenvolveram testes baseados na distância entre densidades espectrais e reamostragens aleatórias para comparar conjuntos de grafos. Para conjuntos de apenas um grafo, FUjiTA, LiRA et al., 2019 desenvolveram um teste semi-paramétrico baseado no estimador de parâmetro de TAKAHASHI et al., 2012, cuja taxa de falsos positivos é controlada pelo nível de significância do teste e o poder estatístico cresce com o número de vértices para diversos modelos de grafo aleatório. Ao ser contrastado com outros testes entre dois ou mais grafos (Tang, Athreya, Sussman, Lyzinski e Priebe, 2017; Tang, Athreya, Sussman, Lyzinski, PArk et al., 2017; Ghoshdastidar et al., 2017), o método de Fujita, Lira et al., 2019 foi o único que controlou a taxa de falsos positivos para grafos livre de escala de Barabási-Albert (BArABÁsi e Albert, 1999). Além disso, foram apresentadas aplicações bem sucedidas dos métodos baseados na densidade espectral para analisar redes de interação proteína-proteína (TAKAHASHi et al., 2012; FujitA, LiRA et al., 2019) e de coativação entre regiões do cérebro (TAKAHASHi et al., 2012; FujitA, M. C. VidAL et al., 2017).

Apesar dos resultados obtidos com a densidade espectral na implementação de métodos estatísticos para grafos e na análise de dados biológicos e dos avanços no estudo da convergência da densidade espectral, pouco se sabe sobre as propriedades teóricas do estimador de parâmetros proposto por TAKAHASHI et al., 2012.

Outro problema de grande interesse para a análise de redes biológicas, particularmente em neurociência, é medir a associação entre grafos (redes funcionais do cérebro) e outros valores observados (volume/área de estruturais cerebrais, medidas cognitivas e comportamentais, etc) a fim de investigar as bases neurais de determinadas características ou doenças. Trata-se de um problema frequente no estudo do Transtorno do Espectro 
1 | INTRODUÇÃO

Autista (TEA), cujas bases neurais são pouco conhecidas. A fim de investigar as bases neurais de dois sub-grupos de TEA (autismo e Asperger), interessa-nos saber como a rede funcional do cérebro muda de acordo com o volume de estruturas de matéria branca. Em particular, nos focamos na rede modo padrão do cérebro, que é frequentemente associada ao TEA (Heuvel e Hulshoff Pol, 2010; Bullmore e Sporns, 2009; Harrison et al., 2007; Rombouts et al., 2009; Garrity et al., 2007; Lowe et al., 2008; Mohammadi et al., 2009; Whitfield-GABRieli et al., 2009; Zhou et al., 2007), e no corpo caloso, que é a maior estrutura de matéria branca do cérebro e está envolvida em processos cognitivos alterados em TEA (Travers et al., 2015).

Nesse contexto, usualmente aplica-se uma regressão/teste de correlação entre o volume da estrutura cerebral (ou outra medida de interesse) e alguma característica da rede, como o peso de cada aresta (que quantifica a coativação entre cada par de regiões do cérebro) (R. A. MAson et al., 2008), o peso médio entre as arestas (JUst et al., 2007; Schipul et al., 2012) e medidas descritivas da estrutura do grafo (SATO et al., 2015). Contudo, as medidas comumente utilizadas não são sempre eficazes para descrever os processos aleatórios que geraram os grafos. Por exemplo, as redes de Watts-Strogatz apresentam um coeficiente de agrupamento elevado como estruturas regulares e caminho característico curto como os grafos de Erdős-Rényi (TAKAHASHi et al., 2012). Além disso, grafos de Watts-Strogatz podem apresentar grau médio constante mesmo quando gerados com diferentes probabilidades de reconectar as arestas.

Supondo que os grafos observados foram gerados por um mesmo modelo de grafo aleatório (mas com diferentes parâmetros), o problema mencionado pode ser pensado como o estudo da relação entre os parâmetros usados para gerar os grafos e uma variável aleatória $X$ de interesse. FujitA, TAKAHAshi et al., 2017 abordaram um problema semelhante em que $X$ corresponde a um conjunto de grafos. Os autores propõem uma medida de correlação e um teste baseado no maior autovalor da matriz de adjacência, que está monotonicamente associado ao parâmetro de alguns modelos de grafo aleatório conhecidos (FujITA, TAKAHASHI et al., 2017). O teste proposto apresentou resultado superior a outros baseados em medidas estruturais do grafo, como transitividade, betweenness, proximidade, autovetor, grau e assortatividade.

Neste trabalho propomos adaptar e melhor compreender o estimador de parâmetros proposto por TAKAHASHi et al., 2012 e a correlação entre grafos de FujITA, TAKAHASHI et al., 2017. Primeiramente, complementamos o estudo de simulação realizado por TAKAHASHI et al., 2012, considerando outros cenários e modelos de grafo aleatório. Em seguida, nós propomos duas variantes do método: uma baseada na norma $\ell_{1}$ entre as densidades espectrais e outra baseada na norma $\ell_{1}$ entre as distribuições acumuladas dos autovalores. Para ambas as variantes nós demonstramos a consistência do estimador de parâmetros quando o limite da densidade espectral (à medida que o tamanho do grafo vai para infinito) existe e é conhecido analiticamente. Por fim, abordamos o problema de correlacionar grafos para investigar a relação entre a rede modo padrão do cérebro e o volume do corpo caloso em Asperger e autismo.

Nós detalhamos os objetivos deste trabalho na Seção 1.1. As contribuições obtidas estão resumidas na Seção 1.2. A notação e as principais definições usadas (medida, grafo aleatório, espectro e densidade) são apresentadas no Capítulo 2. No Capítulo 3, fazemos 
um resumo sobre resultados de convergência da densidade espectral de diversos modelos de grafos aleatórios. No Capítulo 4, apresentamos conceitos de Teoria da Informação para grafos com base na densidade espectral. Descrevemos e avaliamos o método de estimação de parâmetros de TAKAHASHi et al., 2012 e as adaptações propostas (baseadas na norma $\ell_{1}$ ) por meio de simulação no Capítulo 5. Além disso, nesse Capítulo, demonstramos os Teoremas 2 e 3 sobre a consistência dos estimadores propostos baseados nas densidades espectrais e distribuições acumuladas dos autovalores, respectivamente. Em seguida, no Capítulo 7, estudamos a relação entre a rede modo padrão do cérebro e o volume do corpo caloso em Asperger e autismo. Por fim, um resumo das conclusões da tese é apresentado no Capítulo 8.

\subsection{Objetivos}

Os objetivos deste trabalho são:

1. propor adaptações ao estimador de parâmetro de TAKAHASHI et al., 2012;

2. derivar o comportamento assintótico dos estimadores de parâmetro propostos;

3. verificar o desempenho dos estimadores de parâmetro por simulação;

4. ilustrar a aplicação de métodos estatísticos em um grande conjunto de dados de imagem de cérebro chamado ABIDE (Di MARTINo et al., 2014), a fim de verificar a relação entre a rede modo padrão do cérebro e o volume/tamanho de regiões do corpo caloso em indivíduos com Asperger e autismo.

\subsection{Contribuições}

Este trabalho contribui para a melhor integração entre Teoria dos Grafos e Estatística, fornecendo novos conhecimentos sobre métodos estatísticos para grafos aleatórios. Além disso, apresentamos uma aplicação em Neurociência, que poderá auxiliar no estudo de Asperger e autismo e inspirar trabalhos nessa área. A seguir listamos trabalhos relacionados a esta tese.

\subsubsection{Produção de textos técnicos e científicos}

\section{Capítulo de livro publicado}

A partir de experimentos de simulação, validamos os métodos propostos por TAKAHASH et al., 2012 para estimação de modelos, estimação de parâmetro e testes de hipótese baseados na densidade espectral, considerando cenários e modelos de grafo aleatório diversos (além daqueles estudados no trabalho original). Este trabalho, resultou no capítulo de livro:

- Santos et al. (2016) Suzana de Siqueira Santos, Daniel Yasumasa Takahashi, João Ricardo Sato, Carlos Eduardo Ferreira e André Fujita. Statistical methods in graphs: parameter estimation, model selection, and hypothesis test. In: Mathematical foundations and applications of graph entropy. Weinheim: Wiley-VCH; 2016. 
1.2 | CONTRIBUIÇÕES

\section{Artigos publicados}

A partir do conceito de entropia espectral de grafos (baseado na densidade espectral), nós comparamos a rede funcional do cérebro entre controles e indivíduos com autismo, resultando no artigo:

- Sato et al. (2015) João Ricardo Sato, Maciel Calebe Vidal, Suzana de Siqueira Santos, Katlin Brauer Massirer e André Fujita. Complex network measures in Autism Spectrum Disorders. IEEE/ACM Transactions on Computational Biology and Bioinformatics. 2015;PP(99):1-1.

O método de estimação de parâmetro de grafos aleatórios foi aplicado para o desenvolvimento de um teste estatístico para comparar dois ou mais grafos:

- Fujita et al. (2019) Andre Fujita, Eduardo Silva Lira, Suzana de Siqueira Santos, Silvia Yumi Bando, Gabriela Eleuterio Soares e Daniel Yasumasa Takahashi. A semi-parametric statistical test to compare complex networks. Journal of Complex Networks, cnz028, https://doi.org/10.1093/comnet/cnz028.

Em particular, para comparar duas ou mais redes de co-expressão, a partir da densidade espectral do grafo, foi desenvolvido um teste de hipótese e uma ferramenta chamada BioNetStat:

- Jardim et al. (2019) Vinícius Carvalho Jardim, Suzana de Siqueira Santos, André Fujita e Buckeridge Marcos Silveira. BioNetStat: A Tool for Biological Networks Differential Analysis. Frontiers in Genetics. 2019; 10:594.

\section{Artigo submetido}

A análise da relação entre a rede modo padrão do cérebro e o volume do corpo caloso está descrita no trabalho "Increased association between the default-mode network and the volume of the anterior region of the corpus callosum in individuals with Asperger's disorder”, de Suzana de Siqueira Santos, Leonardo Baldaçara, João Ricardo Sato e André Fujita, em estágio de submissão para revista científica.

\section{Artigo em preparação}

Como resultado da revisão de resultados de convergência da densidade espectral e da demonstração da consistência do estimador de parâmetro baseado na norma $\ell_{1}$ entre densidades espectrais e entre distribuições acumuladas, estamos preparando um manuscrito para publicação.

\subsubsection{Software}

Disponibilizamos no CRAN o pacote statGraph (https://CRAN.R-project.org/package= statGraph), com métodos estatísticos para analisar grafos, como estimação de parâmetro, seleção de modelos, teste de hipótese e correlação entre grafos. 



\section{Capítulo 2}

\section{Notação e definições}

Conforme vimos no Capítulo 1, parte deste trabalho é baseada na densidade espectral (distribuição dos autovalores da matriz de adjacência) de grafos aleatórios. Primeiramente, na Seção 2.1, apresentamos algumas definições na área de Probabilidade necessárias à compreensão da densidade espectral. Em seguida, na Seção 2.2, definimos grafos, medida de probabilidade dos autovalores e densidade espectral. Na Seção 2.3, definimos grafos aleatórios e convergência da medida de probabilidade dos autovalores. Por fim, na Seção 2.4, apresentamos a notação assintótica utilizada no trabalho.

\subsection{Probabilidade}

Informalmente, em Probabilidade, dizemos que desejamos "medir" a frequência de ocorrências de eventos. Isto é, estamos interessados no "tamanho" do conjunto que contém as "ocorrências". Para definir uma família de conjuntos "mensuráveis", criou-se o conceito de $\sigma$-álgebra. Além disso, funções como a medida de Lebesgue foram definidas para medir os conjuntos de uma $\sigma$-álgebra. Esses conceitos, bem como outras definições fundamentais em Probabilidade (variáveis aleatórias, distribuição de probabilidade, etc) são apresentados na Seção 2.1.1, com base no livro de CAPINSKi e Kopp, 2004. Nesse contexto, lidamos com integrais com respeito a medidas e interessa-nos saber quando uma integral de uma sequência convergente de funções mensuráveis converge para a integral da função assintótica. Esse assunto é abordado no Teorema de convergência dominada, na Seção 2.1.2 e posteriormente utilizado na demonstração dos Teoremas 2 e 3, no Capítulo 5.

\subsubsection{Definições}

Uma $\sigma$-álgebra $\Sigma$ é uma família de subconjuntos de um conjunto $\Omega$ tal que

1. $\varnothing \in \Sigma$;

2. se $E \in \Sigma \Longrightarrow E^{c} \in \Sigma$;

3. a união contável de elementos de $\Sigma$ está em $\Sigma$.

A interseção de todas as $\sigma$-álgebras que contém $\Omega$ é chamada de álgebra de Borel. 
Um par ordenado $(\mathcal{X}, \Sigma)$, onde $\mathcal{X}$ é um conjunto e $\Sigma$ é uma $\sigma$-álgebra sobre $\mathcal{X}$ é chamado de espaço mensurável. Dados dois espaços mensuráveis $\left(\mathcal{X}_{1}, \Sigma_{1}\right)$ e $\left(\mathcal{X}_{2}, \Sigma_{2}\right)$, a função $f$ é mensurável se, para qualquer conjunto $E \in \Sigma_{2}$, o conjunto $f^{-1}(E):=\left\{x \in \mathcal{X}_{1}: f(x) \in E\right\}$ pertencer a $\Sigma_{1}$.

Uma medida $\mu: \Sigma \rightarrow[0, \infty]$ em $\Omega$ é uma função tal que

1. $\mu(\varnothing)=0$;

2. se $\left(E_{i}\right) \operatorname{com} i \in \mathbb{N}$ é uma sequência disjunta dois a dois em $\Sigma$, então

$$
\mu\left(\bigcup_{i \in \mathbb{N}} E_{i}\right)=\sum_{i=0}^{\infty} \mu\left(E_{i}\right) .
$$

Alguns tipos particulares de medida tornaram-se conhecidos pela sua ampla utilização. Um deles é a medida de Dirac. Dados $a \in \mathbb{R}$ e $E \in \Sigma$, a medida de Dirac centrada em $a$ é definida como

$$
\delta_{a}(E)=\left\{\begin{array}{lll}
1 & \text { se } & a \in E \\
0 & \text { se } & a \notin E .
\end{array}\right.
$$

Seja $E \subseteq \mathbb{R}$. A medida exterior de Lebesgue de $E$ é definida como

$$
m^{\star}(E)=\inf Z_{E},
$$

onde

$$
Z_{E}=\left\{\sum_{i=1}^{\infty} l\left(I_{i}\right): I_{i} \text { são intervalos, } E \subseteq \bigcup_{i=1}^{\infty} I_{n}\right\}
$$

e $l([a, b])=b-a$ é o comprimento do intervalo.

Considere $\Omega=\mathbb{R}$ e $\Sigma=\mathcal{M}$ a $\sigma$-álgebra de Borel sobre os reais tal que

$$
m^{\star}(A)=m^{\star}(A \cap E)+m^{\star}\left(A \cap E^{c}\right)
$$

onde $E^{c}=\mathbb{R} \backslash E$ e $E \in \mathcal{M}$.

A medida de Lebesgue $m: \mathcal{M} \mapsto[0, \infty]$ é definida de forma análoga à medida exterior de Lebesgue, mas é restrita à $\sigma$-álgebra $\mathcal{M}$.

Dada uma medida, podemos definir o conceito de integral sobre essa medida. Primeiramente, consideramos o caso em que queremos integrar um tipo particular de função, que definimos a seguir.

Seja $\Sigma$ uma $\sigma$-álgebra sobre os reais. Uma função não negativa $f: \mathbb{R} \mapsto \mathbb{R}$ que assume um conjunto finito de valores reais não negativos $\left\{a_{1}, a_{2}, \cdots, a_{N}\right\}$ é uma função simples se todos os conjuntos

$$
A_{i}=f^{-1}\left(\left\{a_{i}\right\}\right)=\left\{x: f(x)=a_{i}\right\},
$$

para $i=1,2, \cdots, N$, pertencerem a $\Sigma$. 
A integral de Lebesgue sobre $E \in \mathcal{M}$ da função simples $f$ é dada por

$$
\int_{E} f d m=\sum_{i=1}^{N} a_{i} m\left(A_{i} \cap E\right)
$$

onde $m$ corresponde à medida de Lebesgue e $d m$ pode ser pensada como a integral calculada em um intervalo $d x$.

A integral de Lebesgue de uma função mensurável não-negativa $g$ sobre $E \in \mathcal{M}$ é definida como

$$
\int_{E} g d m=\sup Y(E, g)
$$

onde

$$
Y(E, g)=\left\{\int_{E} f d m: 0 \leq f \leq g, f \text { é uma função simples }\right\} \text {. }
$$

Seja $h$ uma função mensurável em $\mathcal{M}$. Denotamos as partes negativas e positivas de $h$ por $h^{-}$e $h^{+}$, respectivamente. Se $\int_{E} h^{-} d m$ e $\int_{E} h^{+} d m$ são finitas, nós dizemos que $h$ é integrável e definimos

$$
\int_{E} h d m=\int_{E} h^{+} d m-\int_{E} h^{-} d m
$$

Analogamente, podemos definir a integral com respeito a uma medida qualquer $\mu$ substituindo a medida de Lebesgue $m$ por $\mu$ nas fórmulas anteriores.

Dado um espaço mensurável $(\Omega, \Sigma)$, quando existe uma medida $\mu$ definida em $\Sigma$, dizemos que $(\Omega, \Sigma, \mu)$ é um espaço de medida. Se $\mu=P$ for uma medida tal que $P(\Omega)=1$, então $P$ é chamada de medida de probabilidade e $(\Omega, \Sigma, P)$ é chamado de espaço de probabilidade.

Uma variável aleatória $X$ é uma função $X: \Sigma \mapsto \mathbb{R}$ tal que, para todo $a \in \mathbb{R}$, o conjunto $X^{-1}([a, \infty[):=\{\omega \in \Omega: X(\omega) \geq a\}$ está em $\Sigma$.

Dada uma variável $X$, a $\sigma$-álgebra gerada por $X$ é definida como a família de conjuntos $X^{-1}(\mathcal{B})=\left\{S \in \Sigma: S=X^{-1}(B)\right.$ para algum $\left.B \in \mathcal{B}\right\}$, onde $\mathcal{B}$ é a $\sigma$-álgebra de Borel sobre os reais. A distribuição de probabilidade de $X$ é uma medida sobre $\mathcal{B}$ definida como $P_{X}(B)=P\left(X^{-1}(B)\right)$, onde $B \in \mathcal{B}$.

Se a variável aleatória $X: \Omega \mapsto \mathbb{R}$ tem distribuição de probabilidade $P_{X}$, temos

$$
\int_{\Omega} g(X(\omega)) d P(\omega)=\int_{\mathbb{R}} g(x) d P_{X}(x)
$$

Seja $E \in \mathcal{M}$. A medida $P$ da forma

$$
E \mapsto P(E)=\int_{E} f d m
$$

com a função $f$ integrável é chamada de absolutamente contínua e a função $f$ é a densidade de $P$ com respeito à medida de Lebesgue. Sendo $P$ uma probabilidade, temos $\int f d m=$ 1. 
A função acumulada correspondendo à densidade $f$ é dada por

$$
F(x)=\int_{-\infty}^{x} f(x) d x
$$

A esperança de uma variável aleatória $X$ definida no espaço $(\Omega, \Sigma, P)$ é definida como

$$
E(X)=\int_{\Omega} X d P
$$

ou, equivalentemente,

$$
E(X)=\int_{-\infty}^{\infty} x d P_{X}(x)
$$

Se $P$ for absolutamente contínua, então

$$
E(X)=\int_{-\infty}^{\infty} x f_{X}(x) d x
$$

onde $f_{X}$ é a função de densidade de $X$.

\subsubsection{Teorema de convergência dominada}

Lebesgue provou o seguinte teorema clássico sobre convergência de integrais com respeito a uma medida.

Teorema 1. Sejam $(\Omega, \Sigma, \mu)$ um espaço de medida e $E$ um conjunto pertencente a $\Sigma$. Seja $\left(f_{n}\right)$ uma sequência de funções mensuráveis tais que $\left|f_{n}\right| \leq g$ ponto a ponto quase sempre em $E$ para qualquer $n \geq 1$, em que g é integrável sobre E. Se $f=\lim _{n \rightarrow \infty} f_{n}$ pontualmente quase sempre, entãof é integrável sobre $E$ e

$$
\lim _{n \rightarrow \infty} \int_{E} f_{n}(x) d \mu=\int_{E} f d \mu .
$$

\subsection{Grafos}

Um grafo $G=(V, E)$ é um par ordenado de um conjunto de vértices $V=\{1,2, \ldots, n\}$ e um conjunto de arestas $E$ conectando os elementos de $V$. A matriz de adjacência de $G$, denotada por A, é uma matriz $n \times n$ tal que $\mathbf{A}_{i j}=1$ se existir uma aresta entre os vértices $i$ e $j$, e $\mathbf{A}_{i j}=0$, caso contrário, onde $i, j \in V$.

O espectro de $G$ é o conjunto de autovalores de A. Como $G$ não é dirigido, A é uma matriz simétrica e seus autovalores $\lambda_{1}^{G} \geq \lambda_{2}^{G} \geq \ldots \geq \lambda_{n}^{G}$ são reais.

Seja $\delta$ a medida de Dirac. Considere a seguinte medida de probabilidade dos autovalores associada ao grafo $G$ :

$$
\mu^{G}=\frac{1}{n} \sum_{i=1}^{n} \delta_{\lambda_{i}^{G} / \sqrt{n}}
$$


Similarmente, definimos a densidade espectral empírica (DEE) como

$$
\rho^{G}=\frac{1}{n} \sum_{i=1}^{n} 1\left\{x=\lambda_{i}^{G} / \sqrt{n}\right\}
$$

onde 1 é a função indicadora.

A função de distribuição acumulada dos autovalores (DAA) é

$$
F^{G}(x)=\frac{1}{n} \sum_{i=1}^{n} 1\left\{\lambda_{i} \leq x\right\}
$$

Seja $f: \mathbb{R} \mapsto \mathbb{R}$ uma função contínua e limitada. Ao integrar $\mu^{G}$ sobre $f$, obtemos:

$$
\mu^{G}(f)=\int_{\mathbb{R}} f(\lambda) \mu^{G}(d \lambda)=\frac{1}{n} \sum_{i=1}^{n} f\left(\lambda_{i}^{G} / \sqrt{n}\right)
$$

Seja $\left(G_{n}\right)_{n \geq 1}$ uma sequência de grafos sobre o conjunto de vértices $V=\{1,2, \ldots, n\}$, onde $n$ é o número de vértices. Então $\mu^{G_{n}}$ converge fracamente para $\mu$ (e denotamos por $\mu^{G_{n}} \Rightarrow \mu$ ) se, para qualquer função real contínua e limitada $f: \mathbb{R} \mapsto \mathbb{R}$, temos que $\mu(f)=\lim _{n \rightarrow \infty} \mu^{G_{n}}(f)$.

A seguir, iremos tomar um kernel $\phi$ (uma função tal que $\int \phi(x) d x=1$ ) e definir, para cada $x \in \mathbb{R}$, a função $\phi_{x}(\cdot)=\phi(x-\cdot)$. Nesse caso, temos a convolução

$$
x \mapsto \mu^{G_{n}}\left(\phi_{x}\right)=\int_{\mathbb{R}} \phi(x-\lambda) \mu^{G_{n}}(d \lambda)=\frac{1}{n} \sum_{i=1}^{n} \phi\left(x-\lambda_{i}^{G_{n}} / \sqrt{n}\right) .
$$

\subsection{Grafos aleatórios}

Um grafo aleatório é um elemento aleatório (análogo à variável aleatória) que toma seus valores do conjunto de grafos. Um modelo de grafo aleatório é uma coleção finita ou enumerável de grafos, junto com uma distribuição de probabilidade $P$ sobre essa coleção. Seja $\left(\mathcal{G}_{n}\right)_{n \geq 1}$ uma sequência de grafos aleatórios sobre o conjunto de vértices $V=\{1,2, \ldots, n\}$. Então os autovalores de $\mathcal{G}_{n}$ são variáveis aleatórias e $\mu^{\mathcal{G}_{n}}$ é uma medida de probabilidade aleatória. Existem diversas formas nas quais a medida aleatória $\mu^{\mathcal{G}_{n}}$ pode convergir fracamente. Dizemos que $\mu^{\mathcal{G}_{n}}$ converge fracamente na esperança para uma medida de probabilidade $\mu$ se, para toda função contínua e limitada $f: \mathbb{R} \mapsto \mathbb{R}$, nós temos

$$
\mathbb{E} \mu^{\mathcal{G}_{n}}(f) \rightarrow \mathbb{E} \mu(f)
$$

quando $n \rightarrow \infty$. De maneira similar, dizemos que $\mu^{\mathcal{G}_{n}}$ converge fracamente na $P$ probabilidade para uma medida de probabilidade $\mu$ se, para toda função contínua e limitada $f: \mathbb{R} \mapsto \mathbb{R}$ e todo $\epsilon>0$, nós temos

$$
P\left(\left|\mu^{\mathcal{G}_{n}}(f)-\mu(f)\right|>\varepsilon\right) \rightarrow 0,
$$


quando $n \rightarrow \infty$. Se

$$
P\left(\left|\lim _{n \rightarrow \infty} \mu^{\mathcal{G}_{n}}(f)-\mu(f)\right|=0\right)=1
$$

para qualquer $f: \mathbb{R} \mapsto \mathbb{R}$, dizemos que $\mu^{\mathcal{G}_{n}}$ converge fracamente P-quase certamente para $\mu$.

\subsection{Notação assintótica}

Ao longo deste texto, dadas duas funções de $n$, denotadas por $f$ e $g$, escrevemos $f=O(g)$ quando $|f| /|g|$ é limitada pelo numerador à medida que $n \rightarrow \infty$. Dizemos que $f=o(g)$ se $f / g \rightarrow 0$ e que $f=\omega(g)$ se $|f| /|g| \rightarrow \infty$.

Em particular, usamos essa notação quando o parâmetro do modelo de grafo aleatório é uma função do número de vértices e estudamos o comportamento do grafo à medida que $n \rightarrow \infty$. No Capítulo a seguir, nos focamos no estudo da convergência da densidade espectral de grafos aleatórios. 


\section{Capítulo 3}

\section{Densidade espectral de grafos aleatórios}

As propriedades de convergência estudadas neste trabalho dependem do comportamento assintótico da densidade espectral empírica (DEE). Assim, neste Capítulo, fazemos uma revisão de resultados sobre a convergência da DEE de diversos modelos de grafo aleatório.

Para cada DEE, há uma medida de probabilidade dos autovalores $\mu$ associada à densidade. Ao longo do Capítulo, denotamos por $\mu(d x)$ a medida calculada num intervalo infinitesimal com início em $x$. Também dizemos que a DEE converge (em algum sentido) para uma função de densidade fixa se a medida correspondente à primeira convergir para a medida correspondente à segunda.

\subsection{Lei semi-circular de Wigner}

Muitos resultados sobre a distribuição assintótica de autovalores de grafos aleatórios são baseados na lei de Wigner para matrizes aleatórias simétricas. WIGNER, 1958 provou que em matrizes simétricas cujas entradas são variáveis aleatórias reais independentes e identicamente distribuídas, com uma distribuição de probabilidade qualquer, média zero e variância um, a DEE converge fracamente na esperança para uma densidade com medida $\mu_{s c}$, definida como

$$
\mu_{s c}(d x)=\frac{1}{2 \pi} \sqrt{4-x^{2}} \mathbf{1}\{|x| \leq 2\} d x
$$

Alguns anos depois, GrenANDER, 1963 provou que a convergência vale fracamente em probabilidade. Finalmente, ARNOLD, 1967 provou que a DEE converge fracamente quase certamente para a distribuição semi-circular.

\subsection{Erdős-Rényi (ER)}

Um dos modelos mais simples em termos de construção é o modelo proposto por ERDős e RÉNYI, 1959. Dados uma probabilidade $p$ e o número de vértices $n$, cada par de vértices é 
conectado com probabilidade $p$, independentemente dos outros pares.

Sejam $\left(\mathcal{G}_{p, n}\right)_{n \geq 1}$ uma sequência de grafos ER e $\lambda_{1}^{n} \geq \lambda_{2}^{n} \geq \cdots \geq \lambda_{n}^{n}$ os autovalores da matriz de adjacência $A_{n}$ do grafo $\mathcal{G}_{p, n}$. Com base na lei semicircular de Wigner, pode-se provar que se $p=\omega\left(\frac{1}{n}\right)$, então a sequência de medidas normalizadas de $\mathbf{A}_{\mathbf{n}} / \sqrt{n p(1-p)}$, dada por

$$
\tilde{\mu}^{G_{p, n}}=\frac{1}{n} \sum_{i=1}^{n} \delta_{\lambda_{i}^{n} / \sqrt{n p(1-p)}},
$$

converge fracamente quase certamente (e também em probabilidade e na esperança) para a lei semi-circular (Tran et al., 2013). Note que aqui a notação $\tilde{\mu}$ é diferente da utilizada para a medida $\mu$ definida em (2.1), uma vez que a normalização da primeira $(\sqrt{n p(1-p)})$ é diferente da segunda $(\sqrt{n})$.

Se $n p=O(1)$, a convergência não é mais válida.

Uma outra formulação deste resultado diz que se os autovalores forem normalizados por raiz de n, como na Equação (2.1), então a medida de probabilidade dos autovalores, denotada por $\mu^{\mathcal{G}_{p, n}}$, converge fracamente quase certamente para

$$
\mu_{p, s c}(d x)=\frac{1}{2 \pi p(1-p)} \sqrt{4 p(1-p)-x^{2}} \mathbf{1}\{|x| \leq 2 \sqrt{p(1-p)}\} d x
$$

Na Figura 3.1, ilustramos um grafo gerado pelo modelo ER (painel 3.1a) e sua densidade espectral empírica (painel 3.1b).

(a) Grafo ER

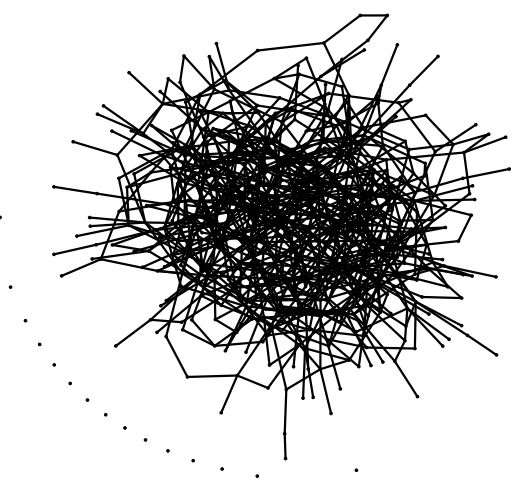

(b) $D E E$

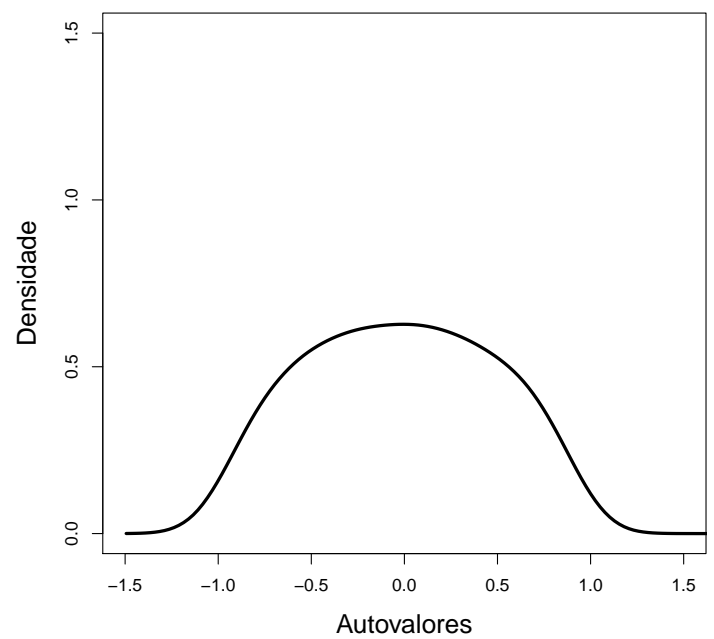

Figura 3.1: (a) Grafo de tamanho 500 gerado pelo modelo Erdős-Rényi e (b) a sua densidade espectral empírica. Figura adaptada de SANTOS et al., 2016. 


\subsection{Modelo bloco estocástico (BE)}

O modelo bloco estocático (BE) (Fienberg e Wasserman, 1981; Holland et al., 1983; SNIJDERs e Nowicki, 1997) produz grafos com grupos de vértices que são conectados entre si com uma densidade de arestas específica. Dados um conjunto de vértices $V=\{1, \ldots, n\}$, cada vértice é atribuído aleatoriamente a um grupo entre $M$ possibilidades $\left\{T_{1}, T_{2}, \ldots, T_{M}\right\}$ independentemente dos demais com probabilidades $\left(\pi_{1}, \pi_{2} \ldots, \pi_{M}\right)$. Então, condicionalmente sobre esses grupos latentes (não-observados), um vértice de $T_{m}$ conecta-se a um vértice de $T_{l}$ independentemente dos demais com probabilidade $p_{m l}$. Note que o grafo aleatório de Erdős-Rényi com parâmetro $p$ é um caso particular do BE onde $p_{m l}=p$, para todo $m, l$.

Aqui nós consideramos um modelo similar. Ao invés de fixarmos as probabilidades $\left(\pi_{1}, \pi_{2} \ldots, \pi_{M}\right)$, nós fixamos o tamanho dos blocos. Esse problema foi abordado por AvRACHENKov et al., 2015, que derivaram uma equação de ponto fixo para a transformada de Stieltjes (QIu e Antonik, 2017) da DEE assintótica. O modelo BE de Avrachenkov e colegas é definido como segue.

Considere um grafo aleatório $\mathcal{G}$ com $n$ vértices $V=\{1,2, \ldots, n\}$ que pertencem a $M$ grupos $\left\{T_{1}, T_{2}, \ldots, T_{M}\right\}$ de tamanho igual $K=n / M$. Assuma que as probabilidades condicionais das conexões são como segue

$$
\mathbb{P}\left(\mathbf{A}_{i j}=1 \mid i \in T_{m}, j \in T_{l}\right)= \begin{cases}p_{0} & \text { se } m \neq l, \\ p_{m} & \text { se } m=l .\end{cases}
$$

Sejam A a matriz de adjacência de $\mathcal{G}$ e $\bar{A}$ sua esperança. Sejam $i \in T_{m}$ e $j \in T_{l}$ dois vértices de $\mathcal{G}$, então a matriz $\overline{\mathrm{A}}$, de dimensões $n \times n$, é tal que se $m=l$, então $\overline{\mathrm{A}}_{i j}=p_{m}$ e, caso contrário, $\overline{\mathrm{A}}_{i j}=p_{0}$. Sejam $p^{\star}=\max _{1 \leq m \leq M} p_{m}$, e $\gamma(n)=1 / \sqrt{n p^{\star}\left(1-p^{\star}\right)}$. A matriz centrada e normalizada de $\mathcal{G}$ é dada por

$$
\tilde{\mathbf{A}}=\frac{(\mathrm{A}-\overline{\mathbf{A}})}{\gamma(n)} .
$$

A expressão para a transformada de Stieltjes da densidade espectral assintótica de $\tilde{A}$ foi obtida por Avrachenkov et al., 2015, como descrito na Proposição 1.

Proposição 1. (AVRACHENKOV et al., 2015). Se $\lim _{n \rightarrow+\infty} n p_{m}=+\infty$ e $p_{m} / p_{0} \leq$ c para alguma constante $c>0$ e para todo $m=1,2, \ldots, M$, então, quase certamente, a distribuição espectral empírica (DEE) converge fracamente para a função de distribuição cuja transformada de Stieltjes é

$$
s(z)=\sum_{m=1}^{M} c_{m}(z),
$$

onde $c_{m}(z)$ é a única solução do sistema de equações

$$
c_{m}(z)=\frac{-1 / M}{z+\zeta_{m} c_{m}(z)+\zeta_{0} \sum_{l \neq m} c_{l}(z)},
$$


com

$$
\zeta_{l}=\lim _{n \rightarrow+\infty} \frac{p_{l}\left(1-p_{l}\right)}{p^{\star}\left(1-p^{\star}\right)}
$$

que satisfaz as condições

$$
\mathfrak{I}\left(c_{l}(z)\right) \mathfrak{I}(z)>0, \text { for } \mathfrak{I}(z)>0,
$$

onde $\mathfrak{I}(z)$ denota a parte imaginária de $z$.

Nós verificamos por meio de simulação se os resultados também podem ser aplicados a matrizes não centradas. Além disso, avaliamos possíveis generalizações dos resultados de Avrachenkov et al., 2015. Assim, nós geramos grafos aleatoriamente e comparamos sua DEE com a densidade teórica derivada por AvRACHENKOv et al., 2015 em quatro cenários diferentes descritos a seguir.

1. Cenário 1. Grafo gerado pelo mesmo modelo descrito por Avrachenkov et al., 2015 com um número pequeno de blocos $(M=3)$. Cada bloco tem tamanho $K=300$. A probabilidade de conectar vértices de grupos diferentes é $p_{0}=0.2$ e as probabilidades dentro dos grupos $\left(p_{1}, p_{2}, p_{3}\right)$ são $(0.8,0.5,0.6)$.

2. Cenário 2. Grafo gerado pelo mesmo modelo descrito por Avrachenkov et al., 2015 com um número maior de blocos $(M=10)$. Cada bloco tem tamanho $K=300$. A probabilidade de conectar vértices de grupos diferentes é $p_{0}=0.2$ e as probabilidades dentro dos grupos $\left(p_{1}, p_{2}, p_{3}, p_{4}, p_{5}, p_{6}, p_{7}, p_{0}, p_{9}, p_{10}\right)$ são $(0.8,0.5,0.6,0.7,0.4,0.9,0.55,0.42,0.38,0.86)$.

3. Cenário 3. Generalização do modelo descrito por Avrachenkov et al., 2015 para blocos de tamanho diferente. Nós consideramos $M=3$ blocos de tamanhos 100 , 80 e 300. A probabilidade de conectar vértices de grupos diferentes é $p_{0}=0.2$ e as probabilidades dentro dos grupos $\left(p_{1}, p_{2}, p_{3}\right)$ são $(0.8,0.5,0.6)$.

4. Cenário 4. Generalização do modelo descrito por Avrachenkov et al., 2015 para $p_{0}$ maior do que as probabilidades dentro dos grupos. Nós consideramos $M=3$ blocos de tamanho $K=300$. A probabilidade de conectar vértices de grupos diferentes é $p_{0}=0.9$ e as probabilidades dentro dos grupos $\left(p_{1}, p_{2}, p_{3}\right)$ são $(0.8,0.5,0.6)$.

A DEE da matriz centrada Ã e da matriz não centrada $\mathbf{A} / \gamma(n)$ são comparadas com a densidade espectral derivada por Avrachenkov et al., 2015 na Figura 3.2. A densidade é computada numericamente a partir da inversão da transformada de Stieltjes. 
(a) Cenário 1

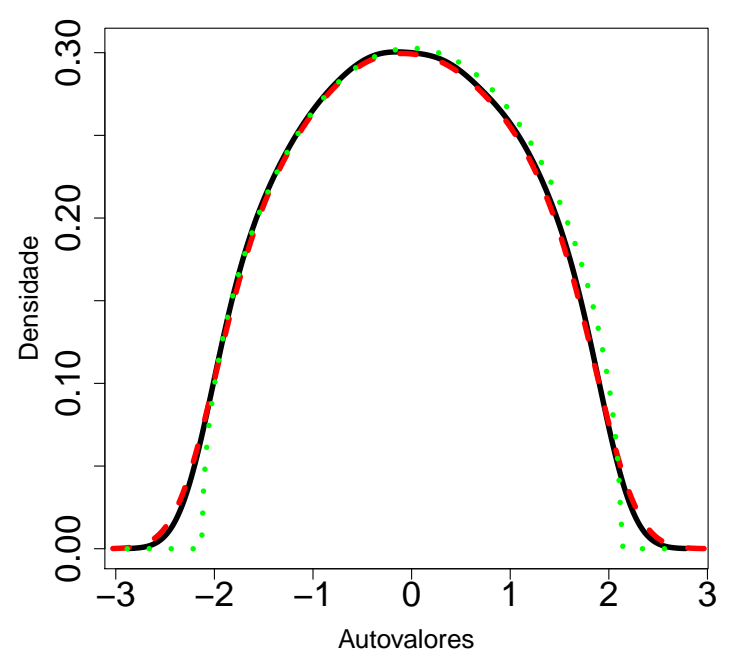

(c) Cenário 3

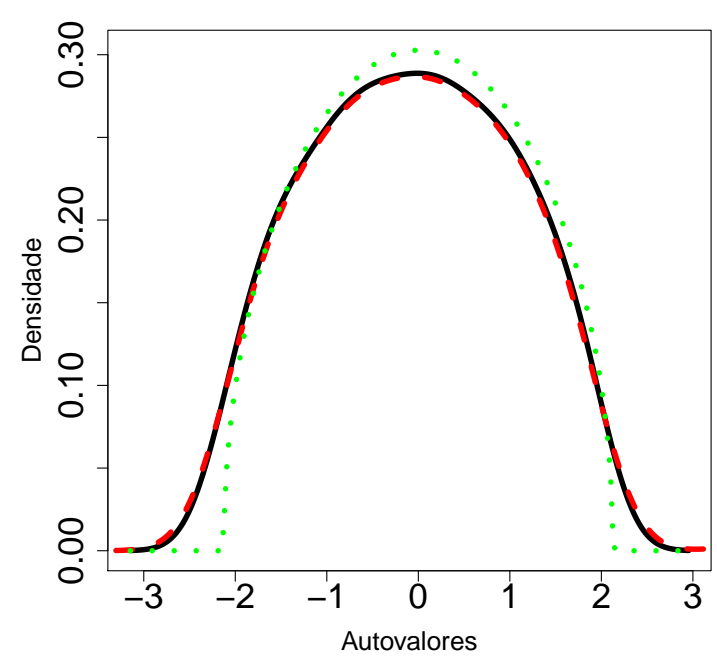

(b) Cenário 2

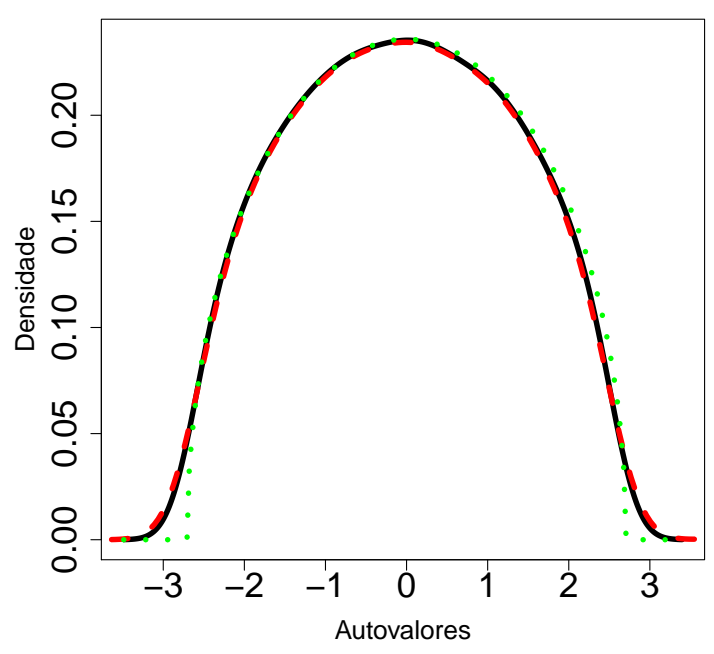

(d) Cenário 4

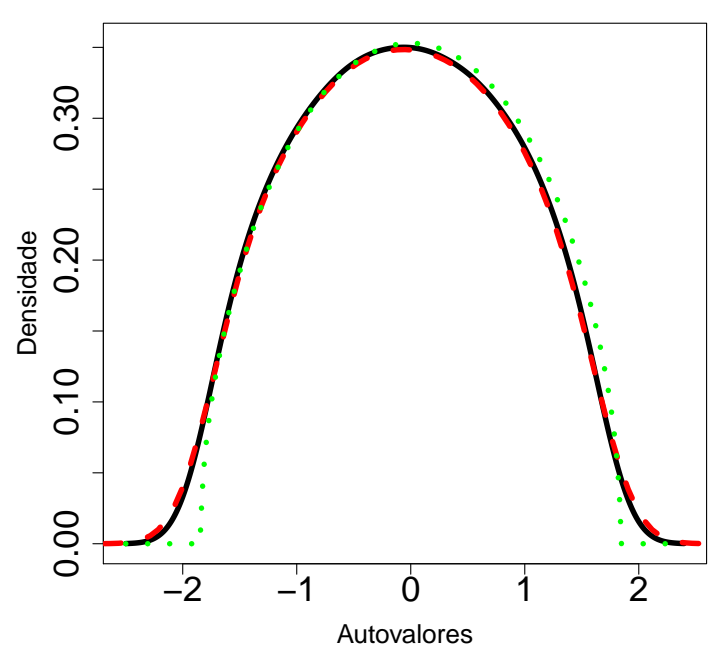

DEE de $\tilde{\mathbf{A}}$

- DEE de $\mathbf{A} / \gamma(n)$

... Densidade teórica

Figura 3.2: Comparação entre a distribuição espectral empírica (DEE) de ̃̃ (linha preta), A/ $\gamma(n)$ (linha vermelha) e a densidade teórica do modelo bloco estocástico derivada por AVRACHENKOV et al., 2015 (linha verde pontilhada) para (a) cenário 1, (b) cenário 2, (c) cenário 3, e (d) cenário 4.

Para todos os cenários considerados, as DEEs de A/ $\gamma(n)$ e Ã ficaram próximas, sugerindo que devem convergir para a mesma distribuição.

Para os cenários 1 , 2, e 4, as distribuições empíricas de $\tilde{A}$ e $\mathbf{A} / \gamma(n)$ são próximas da 
distribuição teórica derivada por AvRACHENKOv et al., 2015. Então, possíveis generalizações dos resultados de Avrachenkov e colegas a serem investigadas incluem modelos com diferentes probabilidades de conectar vértices de grupos diferentes e com um valor grande para $p_{0}$ (em comparação a $p_{m}$ ). Contudo, no cenário 3 , a densidade empírica ficou um pouco longe da densidade teórica, indicando que o resultado de AvRACHenkov et al., 2015 pode não ser aplicável a modelos de blocos de tamanhos diferentes.

\subsection{Grafo aleatório $d$-regular (DR)}

Um grafo $d$-regular é um grafo aleatório sobre $n$ vértices cujos graus são iguais a $d$. Se $d$ é fixo e $\left(\mathcal{G}_{d, n}\right)_{n \geq 1}$ é uma sequência de grafos aleatórios $d$-regulares com matriz de adjacência $\mathbf{A}_{n}$, então a sequência de medidas definidas como

$$
\tilde{\mu}^{\mathcal{G}_{d, n}}=\frac{1}{n} \sum_{i=1}^{n} \delta_{\lambda_{i}^{n}}
$$

converge fracamente quase certamente para

$$
\mu_{d}(d x)=\frac{d \sqrt{4(d-1)-x^{2}}}{2 \pi\left(d^{2}-x^{2}\right)} \mathbf{1}\{|x| \leq 2 \sqrt{d-1}\} d x .
$$

Note que $\tilde{\mu}^{\mathcal{C}_{d, n}}$ é a distribuição dos autovalores de $\mathbf{A}_{n}$, sem dividir por $\sqrt{n}$. Este resultado foi obtido por McKaY, 1981. Ele também é válido quando $d$ cresce lentamente com $n$ (Dumitriu e PAL, 2012; Tran et al., 2013). A distribuição $\mu_{d}$ é conhecida como distribuição Kesten-McKay.

O resultado pode ser reformulado da seguinte forma (BAUERSCHMIDT et al., 2017): a medida de probabilidade dos autovalores da matriz $\mathrm{A}_{\mathrm{n}} / \sqrt{d-1}$ converge fracamente quase certamente para

$$
\tilde{\mu}_{d}(d x)=\left(1+\frac{1}{d-1}-\frac{x^{2}}{d}\right)^{-1} \frac{\sqrt{4-x^{2}}}{2 \pi} \mathbf{1}\{|x| \leq 2 d x\} .
$$

Dumitriu e PAL, 2012 mostraram que a medida de probabilidade dos autovalores $\mathrm{A}_{\mathrm{n}} / \sqrt{d-1}$ converge em probabilidade para $\mu_{s c}$ com $d$ indo para o infinito lentamente, $d=n^{o(1)}$.

Seja $\mathbf{J}_{\mathrm{n}}$ uma matriz $n \times n$ contendo apenas uns, e defina

$$
\mathbf{M}_{\mathbf{n}}=\frac{1}{\sqrt{\frac{d}{n}\left(1-\frac{1}{n}\right)}}\left(\mathbf{A}_{\mathbf{n}}-\frac{d}{n} \mathbf{J}_{\mathbf{n}}\right)
$$

TRAN et al., 2013 mostraram que a DEE de $\mathbf{M}_{\mathbf{n}}$ converge fracamente quase certamente para a distribuição semicircular $\left(\mu_{s c}\right)$ quando $d$ vai para infinito com $n$.

Na Figura 3.3, ilustramos um grafo gerado pelo modelo DR (painel 3.3a) e sua densidade espectral empírica (painel 3.3b). 
(a) Grafo $D R$

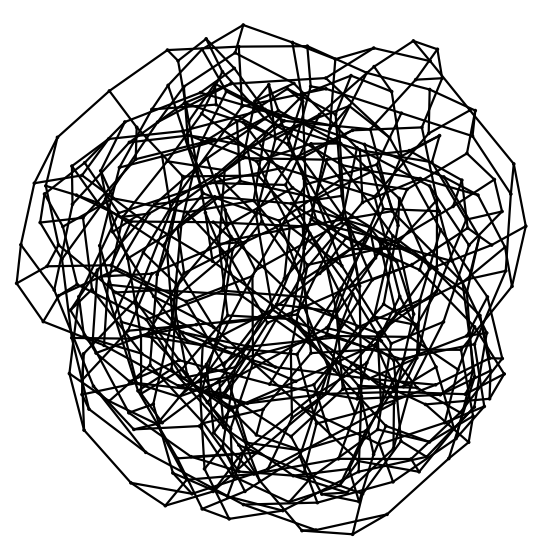

(b) $D E E$

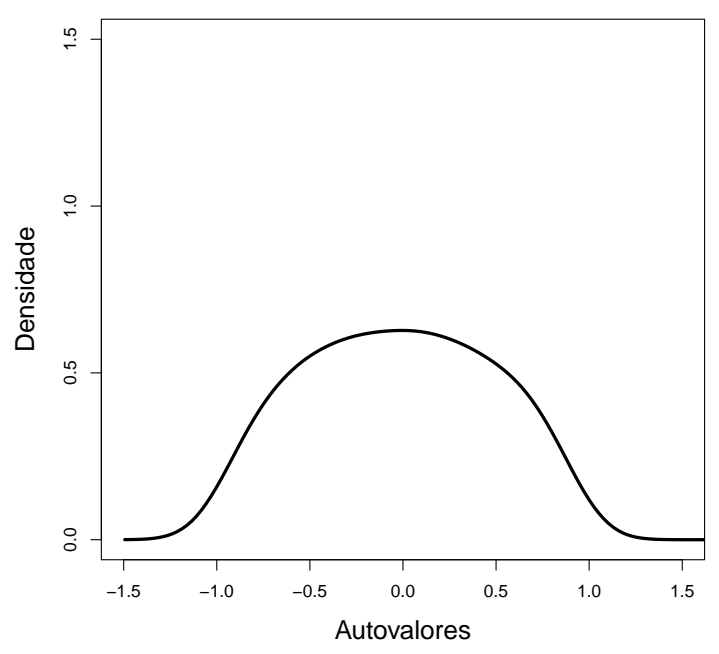

Figura 3.3: (a) Grafo de tamanho 500 gerado pelo modelo d-regular e (b) a sua densidade espectral empírica. Figura adaptada de SANTOS et al., 2016.

\subsection{Modelo geométrico (GE)}

No modelo de grafo aleatório geométrico, grafos são construídos aleatoriamente desenhando-se vértices em um cubo unitário de dimensão $k$ e conectando-se os pares de vértices cujas distâncias são no máximo um dado raio $r$.

Dada uma sequência de grafos aleatórios geométricos com $n$ vértices e raio $r_{n}$, BLACKWELl et al., 2007 mostraram que a densidade espectral (da matriz de adjacência não normalizada) converge fracamente quase certamente para um limite quando $\left.n r_{n}^{k} \rightarrow c \in\right] 0, \infty$ [ à medida que $n \rightarrow \infty$, e que esse limite tem singularidades devido a autofunções localizadas associadas a estruturas presentes no grafo. HAMIDOUCHE et al., 2019 provaram que quando $\left.\left(\frac{n}{\log n}\right) r_{n}^{k} \rightarrow c \in\right] 0, \infty$ [ à medida que $n \rightarrow \infty$ e sob certas condições, a DEE assintótica converge para a densidade espectral da matriz de adjacência de um grafo geométrico determinístico com vértices desenhados sobre uma grade.

Na Figura 3.4, ilustramos um grafo gerado pelo modelo GE (painel 3.4a) e sua densidade espectral empírica (painel 3.4b). 
(a) Grafo GE

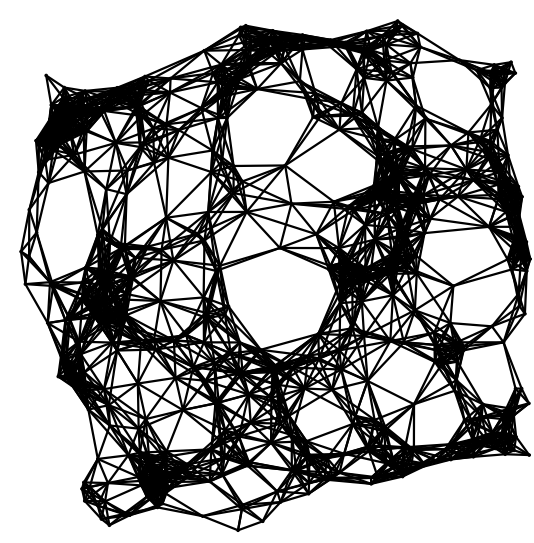

(b) $D E E$

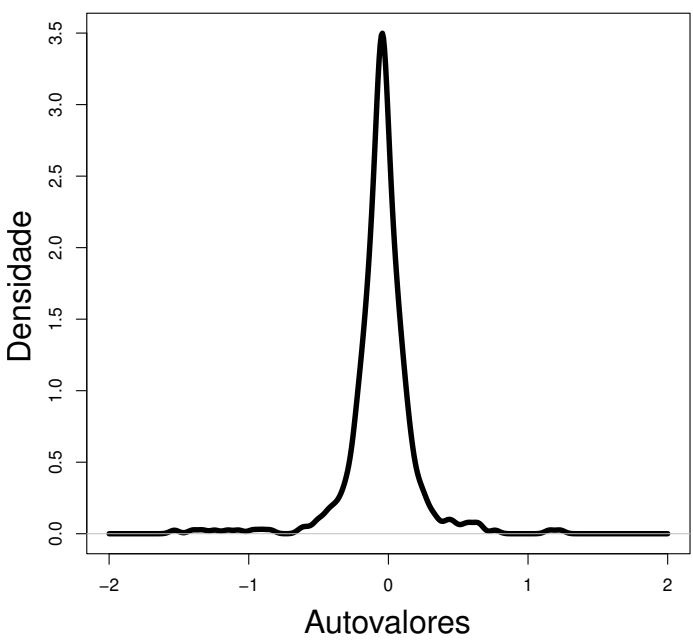

Figura 3.4: (a) Grafo de tamanho 500 gerado pelo modelo geométrico e (b) a sua densidade espectral empírica. Figura adaptada de SANTOS et al., 2016.

\subsection{Modelo de Watts-Strogatz (WS)}

Os grafos aleatórios de Watts-Strogatz (WS) (WATts e Strogatz, 1998) apresentam propriedades de mundo pequeno, como distância média entre os vértices curta (a maioria dos vértices podem ser alcançados a partir de todos os outros vértices com um número pequeno de passos) e um coeficiente agrupamento/clustering (número de triângulos do grafo) maior que o do modelo ER. O processo que gera grafos WS começa com anel regular de tamanho $n$, com cada vértice conectado aos $K / 2$ vizinhos mais próximos em cada lado do anel. Para cada vértice $i$ e cada aresta conectada a $i$, com probabilidade $p_{r}$, a aresta é substituída por uma nova conectando $i$ a um vértice escolhido ao acaso. Ao melhor de nosso conhecimento, todos os resultados sobre o comportamento assintótico da DEE do WS são empíricos (FARKAS et al., 2001).

Na Figura 3.5, ilustramos um grafo gerado pelo modelo WS (painel 3.5a) e sua densidade espectral empírica (painel 3.5b). 
(a) Grafo DR

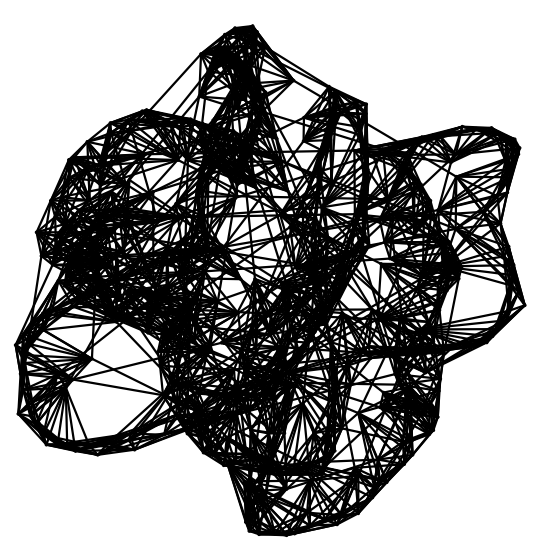

(b) $D E E$

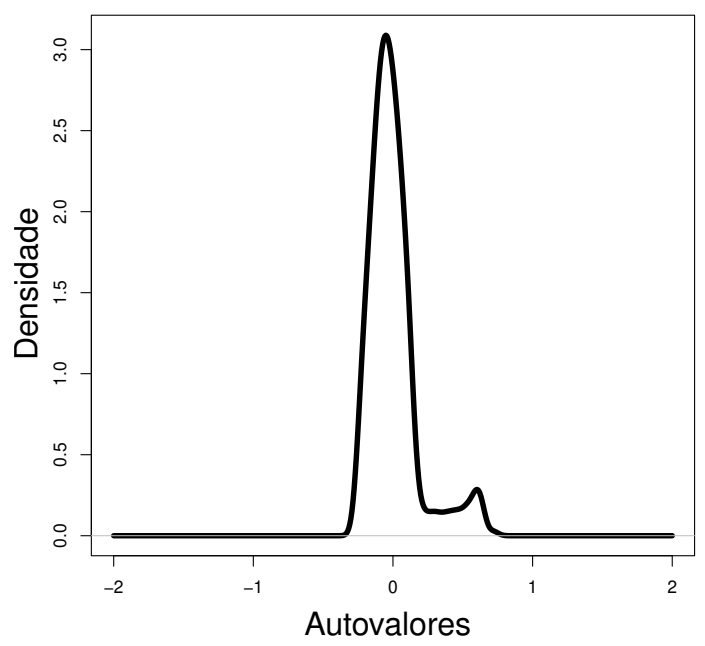

Figura 3.5: (a) Grafo de tamanho 500 gerado pelo modelo Watts-Strogatz e (b) a densidade espectral empírica. Figura adaptada de SANTOS et al., 2016.

\subsection{Modelo de configuração}

O grafo aleatório de configuração tem 2 versões principais na literatura. Seja $\underline{\tilde{d}}=$ $\left(\tilde{d}_{1}, \ldots, \tilde{d}_{n}\right)$ a sequência dos graus do grafo.

O modelo de grau fixo $F D(\underline{\tilde{d}})$ é a coleção de todos grafos com essa sequência de graus, junto com uma distribuição de probabilidade uniforme sobre essa coleção. Esse modelo é obtido por simulação: comece com um grafo real a partir de uma sequência de graus e use um algoritmo de religação de arestas para embaralhá-las.

O modelo de grau aleatório $R D(\underline{\tilde{d}})$ considera todos grafos sobre $n$ vértices tais que as entradas $\mathbf{A}_{i j}$ são independentes com distribuição Bernoulli (não idêntica) com probabilidade de sucesso igual a $p_{i j}$, onde $p_{i j}=\tilde{d}_{i} \tilde{d}_{j} / C$ e $C>0$ é uma constante de normalização escolhida de forma que $0 \leq p_{i j} \leq 1$ (por exemplo, considere $C=\max _{i \neq j} \tilde{d}_{i} \tilde{d}_{j}$ ). Nesse modelo, os grafos tem grau apenas aproximadamente dados por $\underline{\tilde{d}}$. De fato, a esperança do grau do vértice $i$ é

$$
\mathbb{E}\left(d_{i}\right)=\sum_{j \neq i} \mathbb{E}\left(\mathbf{A}_{i j}\right)=\sum_{j \neq i} p_{i j}=\frac{\tilde{d}_{i}}{C} \sum_{j \neq i} \tilde{d}_{j}=\frac{\tilde{d}_{i}\left(2|E|-\tilde{d}_{i}\right)}{C} .
$$

Quando $\tilde{d}_{i}$ não é muito grande e $C \simeq 2|E|$, nós temos $\mathbb{E}\left(d_{i}\right) \simeq \tilde{d}_{i}$.

Seja $\mathrm{A}^{\prime}$ a matriz de adjacência normalizada:

$$
\mathrm{A}^{\prime}=\frac{\mathrm{A}}{\sqrt{n} \sum_{i=1}^{n} \tilde{d}_{i}}
$$

Preciado e RAHimian, 2015 provaram que o modelo de grau aleatório proposto por CHUnG 
e Lu, 2002, sob certas condições a respeito da sequência de graus esperada (esparsidade, momentos finitos, e crescimento controlado dos graus), a DEE da matriz de adjacência normalizada $\mathrm{A}^{\prime}$ converge fracamente quase certamente para uma função de densidade determinística cujos momentos são obtidos explicitamente.

\subsection{Modelo de ligação preferencial}

O modelo de grafo aleatório de ligação preferencial gera grafos adicionando a cada passo um vértice na rede junto com um conjunto da arestas de forma que a probabilidade de conectar o novo vértice seja proporcional ao grau do vértice existente na rede. Um dos modelos de ligação preferencial mais conhecidos é o modelo de Barabási-Albert (BA) (BARABÁsi e AlBERT, 1999), no qual a probabilidade de conectar um vértice a outros vértices existentes é proporcional ao grau elevado ao expoente de escala $p_{s}$. Quando $p_{s} \neq 1$, dizemos que o modelo é não linear. Quanto maior o expoente de escala, maior o peso do grau na probabilidade de conexão e, consequentemente, menor o número de vértices com grau elevado.

Até onde pudemos verificar, não há resultado teórico sobre a convergência da densidade espectral empírica para o modelo de Barabási-Albert, embora o forma da distribuição tenha sido descrita por meio de experimentos de simulação (FARKAS et al., 2001).

Na Figura 3.6, ilustramos um grafo gerado pelo modelo BA (painel 3.6a) e sua densidade espectral empírica (painel 3.6b).

(a) $D E E$

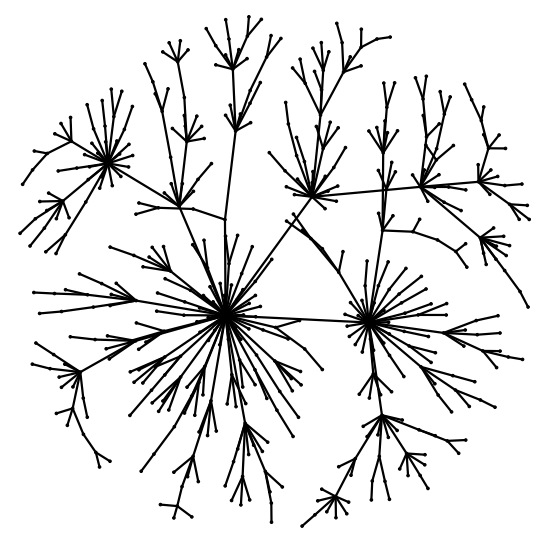

(b) $D E E$

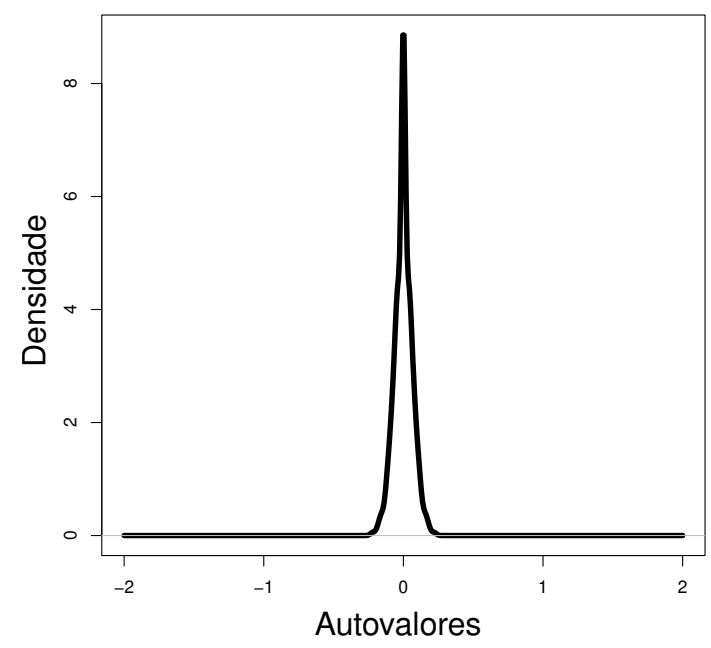

Figura 3.6: (a) Grafo de tamanho 500 gerado pelo modelo Barabási-Albert e (b) a densidade espectral empírica. Figura adaptada de SANTOS et al., 2016.

No modelo de árvore de ligação preferencial linear, a probabilidade de conectar um novo vértice a outros existentes é proporcional ao grau mais $1+a$, onde $a$ é um parâmetro 
do processo (BHAMidi et al., 2012). BhAMidi et al., 2012 provaram que sob certas condições, a DEEs convergem para uma função determinística à medida que $n \rightarrow+\infty$.

\subsection{Conclusões}

Ao revisar as propriedades de convergência das DEEs de grafos aleatórios, nós observamos que a lei semi-circular de Wigner pode ser aplicada aos modelos ER e DR quando as matrizes de adjacência são normalizadas apropriadamente, como mostra a Tabela 3.1. Para o modelo BE, sob algumas condições (blocos de mesmo tamanho, mesma probabilidade de conectar vértices entre grupos diferentes e probabilidade intergrupo menor do que a probabilidade intragrupo), a densidade espectral assintótica da matriz centrada e normalizada pode ser expressa pela transformada de Stieltjes. Além disso, nossos experimentos de simulação sugerem que a convergência das DEEs de matrizes centradas e não-centradas no zero é similar e que elas convergem para a mesma função de densidade obtida pela transformada de Stieltjes quando as probabilidades de conectar vértices de blocos diferentes são distintas e quando a probabilidade intergrupo $p_{0}$ é maior que as probabilidades intragroupos.

\begin{tabular}{cccc}
\hline Modelo & Matriz & $\mu^{\mathcal{G}}$ & Limite \\
\hline ER & $\mathrm{A} / \sqrt{n}$ & $n^{-1} \sum_{i=1}^{n} \delta_{\lambda_{i} / \sqrt{n}}$ & $\mu_{p, s c}(d x)=\frac{1}{2 \pi p(1-p)} \sqrt{\left[4 p(1-p)-x^{2}\right]_{+}} d x$ \\
& $\mathrm{~A} / \sqrt{n p(1-p)}$ & $n^{-1} \sum_{i=1}^{n} \delta_{\lambda_{i} / \sqrt{n p(1-p)}}$ & $\mu_{s c}(d x)=\frac{1}{2 \pi} \sqrt{\left[4-x^{2}\right]_{+}} d x$ \\
\hline \multirow{2}{*}{$D R$} & $\mathrm{~A}$ & $n^{-1} \sum_{i=1}^{n} \delta_{\lambda_{i}}$ & $\mu_{d}(d x)=\frac{d \sqrt{\left[4(d-1)-x^{2}\right]_{+}} d x}{2 \pi\left(d^{2}-x^{2}\right)} d x$ \\
& $\mathrm{~A} / \sqrt{d-1}$ & $n^{-1} \sum_{i=1}^{n} \delta_{\lambda_{i} / \sqrt{d-1}}$ & $\tilde{\mu}_{d}(d x)=\left(1+\frac{1}{d-1}-\frac{x^{2}}{d}\right)^{-1} \frac{\sqrt{\left[4-x^{2}\right]_{+}}}{2 \pi} d x$ \\
\hline $\mathrm{BE}$ & $(\mathrm{A}-\mathrm{E}(\mathrm{A})) / \sqrt{n p^{\star}\left(1-p^{\star}\right)}$ & & expressão por meio da transformada de Stieltjes \\
\hline
\end{tabular}

Tabela 3.1: Convergência da densidade espectral empírica em diferentes modelos.

As DEEs do grafo aleatório geométrico e do modelo de ligação preferencial linear convergem fracamente quase certamente para um limite à medida que $n \rightarrow \infty$. Contudo, não se conhece uma expressão analítica para a função assintótica. Para outros modelos como WS e BA, para o melhor de nosso conhecimento, não há resultados teóricos sobre a convergência de suas DEEs. O conhecimento que se tem sobre seu comportamento foi obtido por meio de experimentos de simulação que sugerem que a DEE desses modelos se aproxima de uma função de distribuição fixa (FARKAS et al., 2001).

A seguir, no Capítulo 4, mostramos uma aplicação da DEE para definir conceitos de Teoria da Informação para grafos. 



\section{Capítulo 4}

\section{Teoria da Informação para grafos}

Vimos no Capítulo anterior que a densidade espectral empírica (DEE) pode descrever alguns modelos de grafo aleatório, mudando de acordo com o parâmetro utilizado para gerar o grafo. Para reforçar o papel análogo da densidade espectral de um grafo aleatório em relação à densidade de uma variável aleatória, mostramos, neste capítulo, como podemos estender conceitos de Teoria da Informação para grafos substituindo a segunda pela primeira. Assim, na Seção 4.1 apresentamos a definição de entropia espectral para grafos. Em seguida, na Seção 4.2, mostramos a definição da medida de divergência de KullbackLeibler para grafos baseada no conceito de entropia.

\subsection{Entropia espectral}

A Teoria da Informação é baseada no conceito de entropia de SHANnon, 1948, que quantifica a quantidade de incerteza de uma variável aleatória. Por exemplo, se uma variável aleatória $X$ segue uma distribuição de Bernoulli com probabilidade de sucesso $p=0.5$, a quantidade de incerteza é a maior possível. Já, se $p=0.9$, temos menos incerteza sobre o valor $X$, pois a probabilidade de sucesso é maior do que a de fracasso.

A quantidade de incerteza (ou "entropia") associada a um evento $\left\{X=x_{i}\right\}$ é definida como $h\left(p_{i}\right)=-\log \left(p_{i}\right)$, onde $p_{i}$ denota a probabilidade de $X$ assumir o valor $x_{i}$. A Entropia de Shannon, 1948 de uma variável discreta $X$ com função de distribuição $p$, que assume os valores $\left\{x_{1}, \ldots, x_{n}\right\}$, é a quantidade de incerteza de $X$, ou a incerteza média de $\left\{x_{1}, \ldots, x_{n}\right\}$, definida como

$$
H(p)=\sum_{i=1}^{n} p_{i} h\left(p_{i}\right)=-\sum_{i=1}^{n} p_{i} \log \left(p_{i}\right) .
$$

Se $X$ for uma variável contínua, então, a incerteza associada a um evento $x$ é obtida a partir da função de densidade $f_{X}(x)$, isto é $h\left(f_{X}(x)\right)=-\log \left(f_{X}(x)\right)$. Além disso, no lugar do somatório, temos a integral sobre os reais: 


$$
H\left(f_{X}\right)=-\int_{\mathbb{R}} f_{X}(x) \log \left(f_{X}(x)\right) d x .
$$

Diferentemente do caso discreto, a entropia $H\left(f_{X}\right)$, como definida acima, pode assumir valores negativos.

Um conceito análogo é a entropia espectral de um grafo aleatório, que quantifica a aleatoriedade da sua estrutura. Seja $\mathcal{G}$ um grafo aleatório e $\rho$ sua densidade espectral com respeito à distribuição de probabilidade de $\mathcal{G}$. A entropia espectral de $\mathcal{G}$ é dada por

$$
H(\rho)=-\int_{-\infty}^{+\infty} \rho(\lambda) \log \rho(\lambda) d \lambda
$$

onde $0 \log 0=0$ (TAKAHASHi et al., 2012).

Para alguns modelos de grafo aleatório, como no modelo de Erdős-Rényi, a densidade espectral assintótica $\rho$ pode ser obtida analiticamente. Nesse caso, em que $\rho$ corresponde à densidade da lei semi-circular, podemos aproximar a entropia espectral para

$$
H(\rho) \sim \frac{1}{2} \ln \left(4 \pi^{2} p(1-p)\right)-\frac{1}{2},
$$

onde $p$ é a probabilidade de conectar um par de vértices (TAKAHASHI et al., 2012). Então o valor máximo da entropia espectral do grafo aleatório de Erdős-Rényi é alcançado quando $p=0.50$. Essa característica é consistente com a ideia intuitiva de que quando todos os possíveis eventos tem a mesma probabilidade de ocorrer, então a capacidade de predizer o sistema é pequena, o que corresponde a uma quantidade de incerteza elevada. Já quando $p \rightarrow 0$ ou $p \rightarrow 1$, a construção do grafo se torna determinística e a quantidade de incerteza associada à estrutura do grafo alcança seu valor mínimo.

A fim de estudar o comportamento da entropia espectral de diferentes modelos de grafos, geramos grafos dos modelos de Erdős-Rényi, $d$-regular, geométrico, Watts-Strogatz e Barabási-Albert a partir do pacote igraph (https://cran.r-project.org/web/packages/ igraph). Para cada modelo e cada valor de parâmetro, a densidade espectral foi estimada a partir de 50 grafos de 500 vértices cada. $O$ valor da entropia espectral obtido para cada grafo aleatório é exibido na Figura 4.1.

Na Figura 4.1a, observamos que, conforme esperado pela expressão analítica da entropia espectral, a entropia estimada para o grafo de Erdős-Rényi é máxima quando $p=0.5 \mathrm{e}$ mínima nos extremos (isto é, quando $p=0$ ou $p=1$ ). Similarmente, o grafo $d$-regular aleatório atinge o valor mínimo quando o grafo se aproxima de um grafo vazio (i.e. quando $d \rightarrow 0$ ), conforme mostramos na Figura 4.1b. Se $d$ aumenta, então a entropia aumenta até $d$ atingir um valor intermediário. Assim nos modelos Erdős-Rényi e $d$-regular, a entropia espectral está associada à noção de quantidade de incerteza do grafo.

No grafo aleatório geométrico, quando o raio $r$ é perto de 0 , a probabilidade de conectar dois vértices é pequena e o grafo provavelmente será vazio. Já quando o parâmetro $r$ é perto de $\sqrt{2}$ o grafo provavelmente se aproximará de um grafo completo. Nesses cenários, espera-se que a quantidade de incerteza seja pequena. De fato, podemos observar na 
Figura 4.1c que a entropia é mínima quando o raio atinge os valores extremos. Já quando o raio $r$ assume valores intermediários, a entropia espectral (ou a quantidade de incerteza) é maior (como mostra a Figura 4.1c). Assim, no modelo de grafo geométrico, a entropia espectral também está associada à quantidade de incerteza do grafo.

A mesma associação é válida para os modelos Barabási-Albert e Watts-Strogatz. Na Figura 4.1d, notamos que a entropia espectral do modelo de Watts-Strogatz cresce com o aumento do parâmetro $p_{r}$, onde $p_{r}$ é a probabilidade de substituir uma aresta, que conecta um vértice $i$ a um vértice próximo a ele na estrutura de anel, pela aresta que conecta $i$ a outro vértice escolhido aleatoriamente. Assim, quando $p_{r}=1$, teremos um grafo construído de forma totalmente aleatória (equivalente ao grafo de Erdős-Rényi) e a quantidade de incerteza será pequena. Já quando $p_{r}=0$, o grafo será determinado pela estrutura de anel e a quantidade de incerteza será mínima. Como ilustrado na Figura 4.1e, a entropia espectral estimada do modelo de Barabási-Albert é inversamente proporcional ao expoente de escala $\left(p_{s}\right)$. Quando $p_{s}$ é pequeno, a aleatoriedade do grafo é alta, uma vez que a influência dos graus dos vértices sobre a probabilidade de se conectar dois vértices é pequena. Já quando $p_{s}$ assume um valor elevado, os graus dos vértices contribuirão fortemente para a inserção de arestas e, assim, a quantidade de incerteza será pequena.

(a) Modelo de Erdős-Rényi

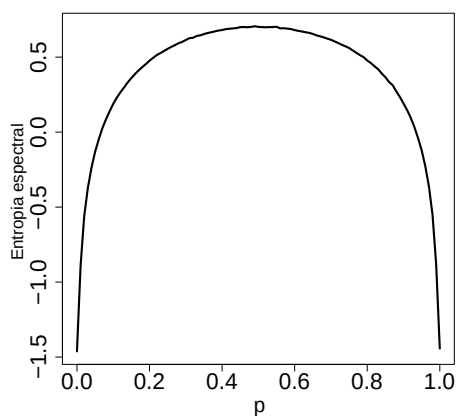

(b) Modelo de grafo d-regular

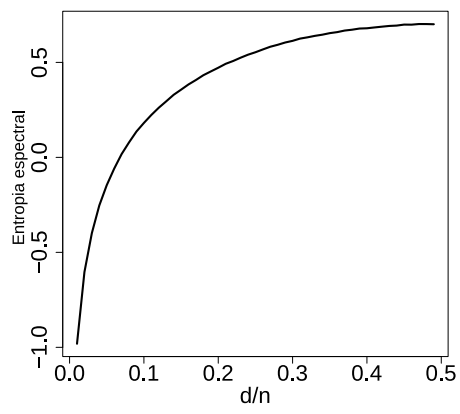

(c) Modelo de grafo geométrico

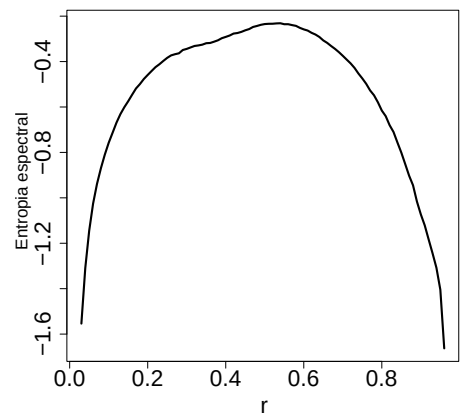

(d) Modelo de Watts-Strogatz

(e) Modelo de Barabási-Albert
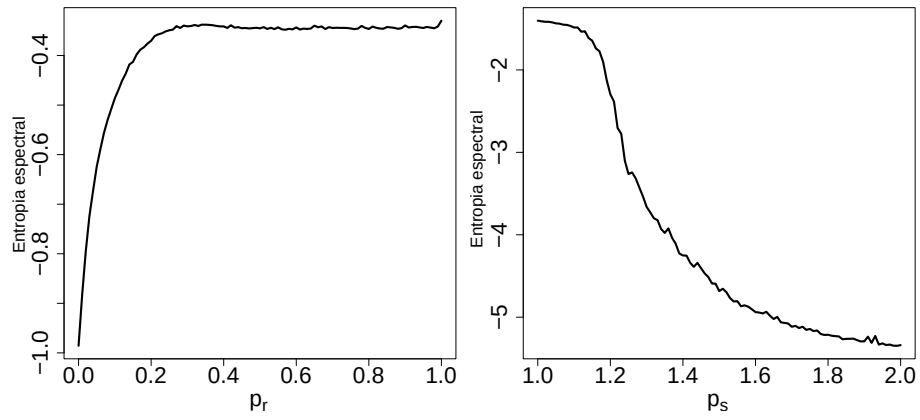

Figura 4.1: Entropia espectral empírica do grafo para os grafos aleatórios de (a) Erdös-Rényi, (b) $d$-regular, (c) geométrico, (d) Watts-Strogatz e (e) Barabási-Albert. O eixo y corresponde à entropia espectral e o eixo $x$ aos parâmetros $p$ (probabilidade de conectar dois vértices), $d / n$ (grau do grafo regular dividido pelo tamanho do grafo), $r$ (raio do modelo de grafo geométrico), $p_{r}$ (probabilidade de reconectar uma aresta) e $p_{s}$ (expoente de escala) em (a), (b), (c), (d) e (e), respectivamente. Para cada modelo, a densidade espectral empírica foi obtida a partir de 50 grafos de tamanho $n=500$. 


\subsection{Divergência de Kullback-Leibler}

A divergência de Kullback-Leibler (KL) mede o aumento de incerteza quando uma distribuição de probabilidade é usada para aproximar outra distribuição. Podemos defini-la em termos dos conceitos de entropia e entropia cruzada.

A entropia cruzada mede a quantidade de incerteza quando uma distribuição $\bar{p}$ é usada para estimar a distribuição verdadeira $p$. Podemos defini-la como

$$
H_{p}(\bar{p})=\sum_{i=1}^{n} p_{i} h\left(\bar{p}_{i}\right)=-\sum_{i=1}^{n} p_{i} \log \left(\bar{p}_{i}\right) .
$$

Assim, a divergência de KL é dada por

$$
K L(p, \bar{p})=H_{p}(\bar{p})-H(p) .
$$

Para grafos, a divergência de KL pode ser usada para medir a dissimilaridade entre grafos aleatórios, selecionar um modelo de grafo aleatório e estimar os parâmetros que melhor descrevem o grafo observado. Claramente, se duas densidades espectrais são diferentes, então os grafos aleatórios correspondentes são diferentes. Contudo, grafos aleatórios diferentes podem ter a mesma densidade espectral (por exemplo, nos grafos ER e DR, a DEE pode convergir para a lei semi-circular).

Sejam $\mathcal{G}_{1}$ e $\mathcal{G}_{2}$ dois grafos aleatórios com densidades espectrais $\rho_{1}$ e $\rho_{2}$, respectivamente. Se o suporte de $\rho_{2}$ contém o suporte de $\rho_{1}$, então a divergência de Kullback-Leibler (KL) entre $\rho_{1}$ e $\rho_{2}$ é

$$
K L\left(\rho_{1} \mid \rho_{2}\right)=\int_{-\infty}^{+\infty} \rho_{1}(\lambda) \log \frac{\rho_{1}(\lambda)}{\rho_{2}(\lambda)} d \lambda
$$

onde $0 \log 0=0$ e $\rho_{2}$ é a medida de referência (TAKAHASHi et al., 2012). Se o suporte de $\rho_{2}$ não contém o suporte de $\rho_{1}$, então $K L\left(\rho_{1} \mid \rho_{2}\right)=+\infty$.

A divergência de KL é não-negativa e vale zero se e somente se $\rho_{1}$ e $\rho_{2}$ são iguais. Note que em muitos casos $K L\left(\rho_{1} \mid \rho_{2}\right)$ e $K L\left(\rho_{2} \mid \rho_{1}\right)$ são diferentes quando $\rho_{1} \neq \rho_{2}$, isto é, a divergência de $K L$ é assimétrica.

No Capítulo a seguir mostramos uma aplicação da divergência de KL entre densidades espectrais para estimar o parâmetro de grafos aleatórios. Além disso, apresentamos versões alternativas do método substituindo a divergência de KL pela norma $\ell_{1}$. 


\section{Capítulo 5}

\section{Estimadores de parâmetro baseados na distribuição dos autovalores}

Neste Capítulo, abordamos o problema de estimar parâmetros de modelos de grafo aleatório. Isto é, dado um grafo e um modelo, queremos encontrar o conjunto de parâmetros que maximize a probabilidade do modelo ter gerado o grafo.

Se considerarmos um grafo gerado pelo modelo ER, podemos obter o estimador de máxima verosimilhança para o parâmetro $p$ a partir da razão entre o número de arestas presentes no grafo e o número total de arestas possíveis $\left(\begin{array}{l}n \\ 2\end{array}\right)$. Contudo, para modelos de grafo aleatório mais complexos em termos de construção, como o WS e o BA, o estimador de máxima verosimilhança não é conhecido.

Nesse caso, é possível estimar a dissimilaridade entre o grafo observado e o grafo gerado por determinado modelo e conjunto de parâmetros e estudar quais valores do parâmetro minimizam a estimação. Com base nessa ideia, TAKAHAshi et al., 2012 propuseram um método para estimação de parâmetro em que a dissimilaridade entre grafos é medida por meio da divergência de Kullback-Leibler entre densidades espectrais.

Na Seção 5.1, apresentamos uma descrição formal do problema de estimação de parâmetros e da abordagem de TAKAHASHi et al., 2012. Em seguida, nas Seções 5.2 e 5.3, estudamos o comportamento do procedimento de TAKAHASHI et al., 2012 e propomos adaptações, respectivamente. Na Seção 5.4, para duas das adaptações apresentadas, derivamos resultados teóricos sobre a convergência dos estimadores de parâmetro, resultando nos Teoremas 2 e 3 . Além disso, mostramos simulações que exemplificam a aplicação dos teoremas e exploram o comportamento dos estimadores tanto em cenários em que as hipóteses sobre a convergência dos densidades espectrais são válidas quanto em cenários em que não há garantia de validade. Finalmente, na Seção 5.5, estudamos empiricamente a distribuição do estimador. 


\subsection{Descrição do método}

Considere um modelo de grafo aleatório paramétrico $\left\{P_{\theta} ; \theta \in \Theta\right\}$ onde $P_{\theta}$ é a distribuição de probabilidade sobre uma coleção de grafos, descrita pelo parâmetro $\theta \in \Theta$, $\Theta \subset \mathbb{R}^{k}$. Seja $\mathcal{G}$ um grafo aleatório de uma distribuição $P$ (que pode ou não pertencer à família paramétrica $\left\{P_{\theta} ; \theta \in \Theta\right\}$ ). Nós queremos estimar um parâmetro $\theta$ tal que $P_{\theta}$ é a mais próxima de $P$. Nós vimos que, em muitos modelos de grafo aleatório, a densidade espectral empírica (DEE) de grafos gerados sob a distribuição $P_{\theta}$ converge fracamente em algum sentido (com respeito a $P_{\theta}$ ) para alguma distribuição que nós denotamos por $\mu_{\theta}$.

As DEEs são distribuições discretas, enquanto que em geral as distribuições assintóticas $\mu_{\theta}$ são medidas absolutamente contínuas (isto é, elas tem densidades com respeito à medida de Lebesgue). Assim, é natural considerar um estimador kernel da densidade do espectro de grafos aleatórios, como descrevemos a seguir. Dada uma função kernel $\phi$ (uma função tal que $\int \phi(x) d x=1$ ), para qualquer $x \in \mathbb{R}$ e qualquer $\sigma>0$, nós denotamos por $\phi_{x, \sigma}(\cdot)$ a função $\sigma^{-1} \phi\left(\frac{x--}{\sigma}\right)$. O estimador kernel da densidade espectral com largura de banda $\sigma$ é definido como

$$
\mu^{\mathcal{G}}\left(\phi_{x, \sigma}\right):=\frac{1}{n \sigma} \sum_{i=1}^{n} \phi\left(\frac{x-\lambda_{i}^{\mathcal{G}} / \sqrt{n}}{\sigma}\right) .
$$

Note que, ao escolher uma largura de banda $\sigma=\sigma_{n}$ que vai para 0 à medida que $n$ vai para infinito, esse estimador deverá se aproximar da densidade espectral assintótica $\mu_{\theta}$ do grafo. Neste trabalho consideramos três critérios para escolha de largura de banda: o critério de Silverman, 1986, o critério de Sturges, 1926 e o critério de validação cruzada não viesada (Scott, 1992; Venables e Ripley, 2003). No critério de Silverman, dada a variância dos autovalores observados $\hat{\sigma}^{2}$, temos a largura de banda

$$
\sigma=\left(\frac{4 \hat{\sigma}^{5}}{3 n}\right)^{\frac{1}{5}}
$$

Já, no critério de Sturges, definimos $n_{\text {bins }}=\left\lceil\log _{2} n+1\right\rceil$ e obtemos a largura de banda

$$
\sigma=\frac{\max _{i} \lambda_{i}^{\mathcal{G}} / \sqrt{n}-\min _{i} \lambda_{i}^{\mathcal{G}} / \sqrt{n}}{n_{\text {bins }}}
$$

Finalmente, no critério de validação cruzada não viesada, minimizamos uma função de erro de estimação, como descrevemos a seguir.

Sejam $\hat{f}(x, \sigma)$ e $\hat{f}_{(-i)}(x, \sigma)$ os estimadores kernel da densidade espectral a partir de todos $n$ autovalores de $\mathcal{G}$ e a partir dos autovalores $\lambda_{j}^{\mathcal{G}}$ tal que $j \neq i$, respectivamente. Mais especificamente, temos $\hat{f}(x, \sigma)=\mu^{\mathcal{G}}\left(\phi_{x, \sigma}\right) \mathrm{e}$

$$
\hat{f}_{(-i)}(x, \sigma):=\frac{1}{(n-1) \sigma} \sum_{j=1, j \neq i}^{n} \phi\left(\frac{x-\lambda_{j}^{\mathcal{G}} / \sqrt{n}}{\sigma}\right) .
$$

Denotando por $\|.\|_{2}^{2}$ a norma $\ell_{2}$ ao quadrado (isto é, dada a função $f$, temos $\|f\|_{2}^{2}=$ 
$\left.\int_{-\infty}^{\infty} \hat{f}(x)^{2} d x\right)$, a função a ser minimizada pelo critério de validação cruzada não viesada é

$$
U C V(\sigma)=\|\hat{f}\|_{2}^{2}-\frac{2}{n} \sum_{i=1}^{n} \hat{f}_{(-i)}\left(\lambda_{i}\right)
$$

Uma vez obtida a largura de banda e as DEEs, o próximo passo é escolher uma medida de dissimilaridade $D$ entre distribuições e, assim, estimar o parâmetro $\theta$ resolvendo o problema de minimização

$$
\hat{\theta}=\underset{\theta \in \Theta}{\operatorname{argmin}} D\left(\mu^{\mathcal{C}}\left(\phi_{\cdot, \sigma}\right), \mu_{\theta}\left(\phi_{\cdot, \sigma}\right)\right)
$$

Na prática, ou usamos uma expressão analítica para $\mu_{\theta}$, ou nos baseamos em estimadores de Monte Carlo. No último caso, nós amostramos $M$ grafos $\mathcal{G}_{1}, \mathcal{G}_{2}, \cdots, \mathcal{G}_{M}$ da distribuição $P_{\theta}$. Depois, para cada grafo $\mathcal{G}_{m}$, nós obtemos $\mu^{\mathcal{G}_{m}}\left(\phi_{x, \sigma}\right)$ numericamente e estimamos $\mu_{\theta}\left(\phi_{x, \sigma}\right)$ como

$$
\hat{\mu}_{\theta}\left(\phi_{x, \sigma}\right)=\frac{1}{M} \sum_{m=1}^{M} \mu^{\mathcal{G}_{m}}\left(\phi_{x, \sigma}\right)=\frac{1}{n M \sigma} \sum_{m=1}^{M} \sum_{i=1}^{n} \phi\left(\frac{x-\lambda_{i}^{\mathcal{G}_{m}} / \sqrt{n}}{\sigma}\right) .
$$

Em suma, o procedimento de estimação de parâmetro segue os passos descritos no Algoritmo 1. 


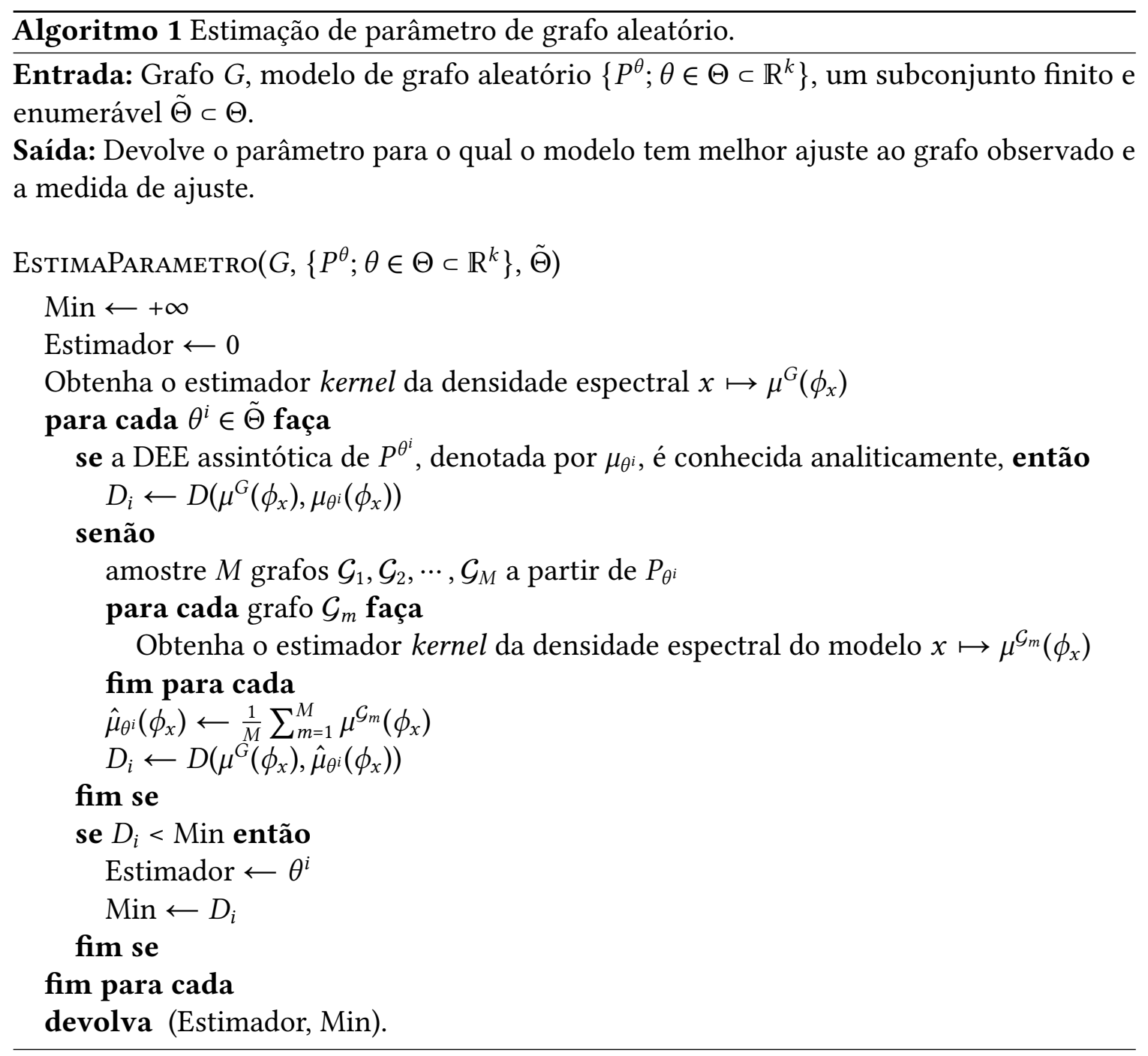

\subsection{Método original}

TAKAHASHi et al., 2012 originalmente propuseram uma abordagem para o problema (5.1) baseada na divergência de Kullback-Leibler, $K L\left(\mu^{\mathcal{G}}\left(\phi_{x}\right), \mu_{\theta}\left(\phi_{x}\right)\right)$, onde $\phi$ é o kernel gaussiano. Neste caso, nós obtemos

$$
D\left(\mu^{\mathcal{G}}\left(\phi_{x, \sigma}\right), \mu_{\theta}\left(\phi_{x, \sigma}\right)\right)=\frac{1}{n \sigma} \sum_{i=1}^{n} \int \phi\left(x-\lambda_{i}^{\mathcal{G}} / \sqrt{n}\right) \log \left(\frac{\sum_{i=1}^{n} \phi\left(x-\lambda_{i}^{\mathcal{G}} / \sqrt{n}\right)}{n \sigma \mu_{\theta}\left(\phi_{x, \sigma}\right)}\right) d x .
$$

Para ilustrar o desempenho do estimador de parâmetro proposto por TAKAHASHI et al., 2012, nós geramos grafos de tamanhos $n=20,50,100,500$ dos modelos Edős-Rényi (ER), $d$ regular (DR), Geométrico (GE), Watts-Strogatz (WS) e Barabási-Albert (BA) com parâmetros $p=0.5, d=0.25 n, r=0.5, p_{r}=0.07$ e $p_{s}=1$. Para o modelo de bloco estocástico (BE), nós geramos grafos de 600 vértices com dois blocos de mesmo tamanho com probabilidade intergrupo $p_{0}=0.2$ e probabilidade intragrupo $p_{1}=0.7$. Nesses experimentos, para cada modelo e cada valor de $n$, geramos 1000 grafos e consideramos o critério de Silverman para estimação da densidade espectral. Na Tabela 5.1, nós podemos ver que o valor médio 
estimado é próximo do valor verdadeiro quando o grafo é suficientemente grande. Similarmente, para o modelo $\mathrm{BE}$, obtemos valores próximos ao esperado, com média \pm desvio padrão de $0.200 \pm 0.001$ para $p_{0}$ e $0.700 \pm 0.002$ para $p_{1}$.

Tabela 5.1: Médias e desvios padrão dos parâmetros estimados pelo método original baseado na divergência de KL entre densidades espectrais para os modelos de grafo aleatório de Erdös-Rényi (ER), d-regular (DR), Geométrico (GE), Watts-Strogatz (WS) e Barabási-Albert (BA). Para cada modelo de grafo aleatório, foram gerados 1000 grafos de tamanhos 20, 50, 100 e 500, aos quais aplicamos o procedimento de estimação de parâmetros. A medida de referência de cada modelo foi estimada a partir da densidade espectral empirica média de 100 grafos gerados pelo modelo. Os parâmetros originais utilizados para gerar os grafos estão indicados entre parênteses ( $p$ é a probabilidade de conectar dois vértices, $d$ é o grau do vértice, $r$ é o raio, $p_{r}$ é a probabilidade de reconectar um vértice e $p_{s}$ é o expoente de escala). O parâmetro d é uma função do número de vértices $n$.

\begin{tabular}{c|cccc}
\hline & \multicolumn{4}{|c}{ Tamanho } \\
Modelo & 20 & 50 & 100 & 500 \\
\hline ER $(p=0.5)$ & $0.506 \pm 0.039$ & $0.501 \pm 0.014$ & $0.501 \pm 0.008$ & $0.499 \pm 0.003$ \\
DR $(d=0.25 n)$ & $0.264 n \pm 0.013 n$ & $0.245 n \pm 0.005 n$ & $0.250 n \pm 0$ & $0.249 n \pm 0.004 n$ \\
GE $(r=0.5)$ & $0.493 \pm 0.061$ & $0.506 \pm 0.037$ & $0.502 \pm 0.022$ & $0.500 \pm 0.010$ \\
WS $\left(p_{r}=0.07\right)$ & $0.129 \pm 0.155$ & $0.069 \pm 0.011$ & $0.071 \pm 0.008$ & $0.070 \pm 0.003$ \\
BA $\left(p_{s}=1\right)$ & $1.128 \pm 0.309$ & $1.044 \pm 0.125$ & $1.026 \pm 0.047$ & $1.020 \pm 0.025$ \\
\hline
\end{tabular}

\subsection{Adaptações}

Nesta Seção, avaliamos a sensibilidade do método proposto por TAKAHASH et al., 2012 ao critério de escolha de largura de banda do estimador da densidade, à função de distribuição dos autovalores e à medida de divergência entre distribuições. Além disso, verificamos adaptações do método que possam melhorar seu desempenho em alguns cenários.

Podemos observar na Tabela 5.1 que o BA é o modelo em que o estimador apresenta maior erro. Assim, geramos 1000 grafos do modelo BA com tamanho 500 e diferentes valores de parâmetro para avaliar o desempenho do procedimento proposto por TAKAHASHI et al., 2012. Quando os grafos são gerados com expoente de escala $p_{s}=1.1$, os valores estimados parecem se concentrar em torno do valor esperado, como vemos na Figura 5.1a. Já quando $p_{s}=1.4$ (Figura 5.1b), há duas modas na distribuição, sugerindo que o estimador não estaria se aproximando do valor esperado.

Uma possível explicação para a diferença de desempenho entre os cenários considerados $\left(p_{s}=1.1\right.$ e $\left.p_{s}=1.4\right)$ são erros de estimação da densidade espectral. Assim nós avaliamos o desempenho do procedimento de TAKAHASH et al., 2012 utilizando diferentes critérios para a escolha da largura de banda da densidade espectral: o critério de Silverman, 1986, o critério de STURGES, 1926 e o critério de validação cruzada não viesada (SCOTT, 1992; Venables e Ripley, 2003). Foram gerados 1000 grafos BA com parâmetro $p_{s}=1.4 \mathrm{e}$ tamanho 500. Os resultados são exibidos na Figura 5.2, onde os boxplots de nomes DEE-KL, DEE-St-KL e DEE-vc-KL se referem, respectivamente, aos critérios de Silverman, Sturges e validação cruzada não viesada. 
(a) $p_{s}=1.1$

Estimador DEE-KL - Histograma

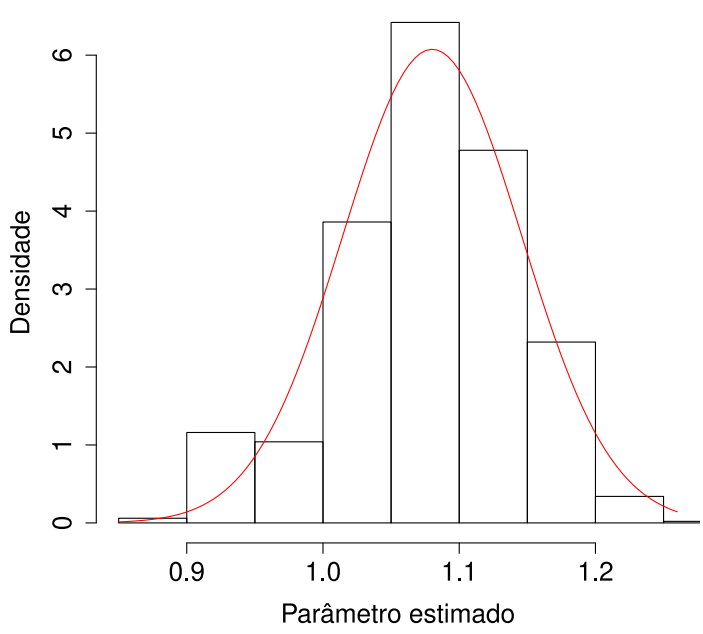

(b) $p_{s}=1.4$

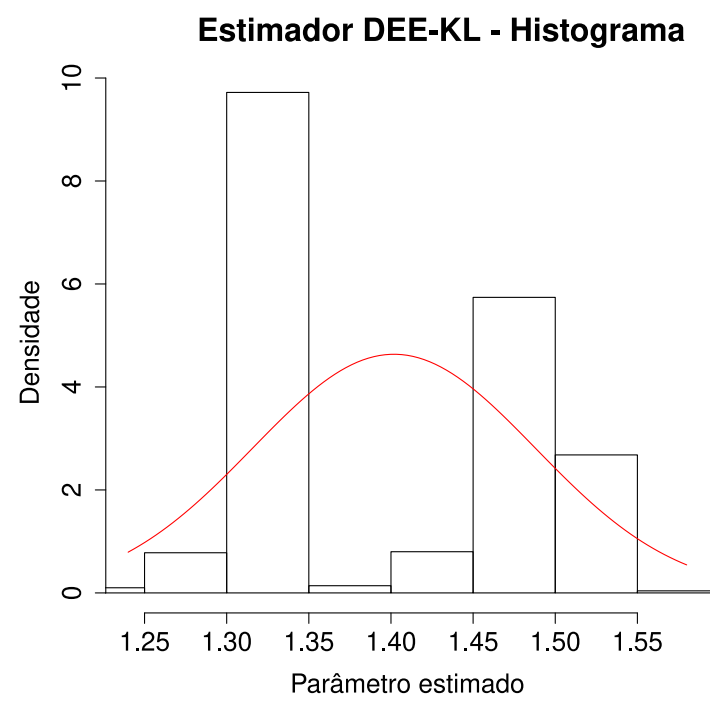

Figura 5.1: Distribuição do estimador de parâmetro baseado na divergência de Kullback-Leibler entre densidades espectrais estimadas pelo critério de Silverman. Cada gráfico corresponde ao histograma do estimador de parâmetro aplicado a 1000 grafos do modelo Barabási-Albert com grafos de tamanho $n=500$ e expoente de escala $(a) p_{s}=1.1 \mathrm{e}(\mathrm{b}) p_{s}=1.4$. A linha vermelha corresponde à função da densidade da distribuição normal ajustada aos autovalores.

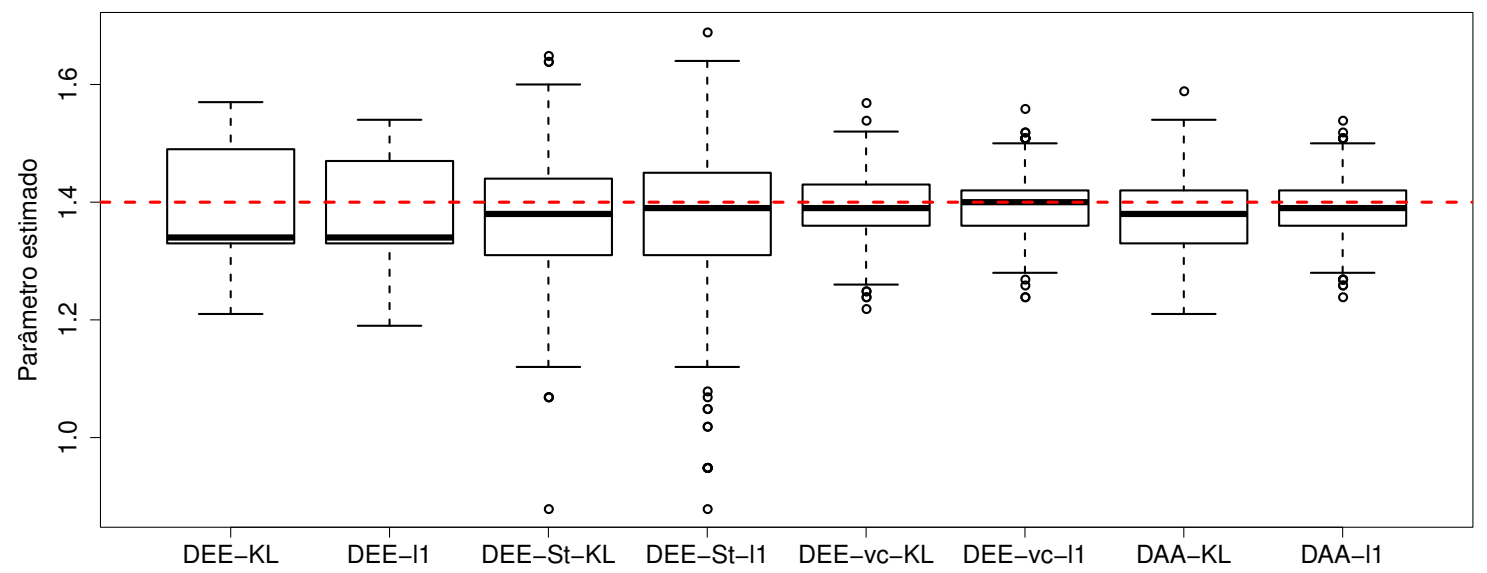

Figura 5.2: Boxplots dos estimadores de parâmetro para grafos BA de tamanho $n=500 \mathrm{com}$ expoente de escala $p_{s}=1.4$. Cada boxplot é nomeado pelo método utilizado para ajustar o modelo: DEE-KL (divergência de Kullback-Leibler entre DEEs estimadas com o critério de Silverman), DEE-l1 (norma $\ell_{1}$ entre DEEs estimadas com o critério de Silverman), DEE-St-KL (divergência de Kullback-Leibler entre DEEs estimadas com o critério de Sturges), DEE-St-l1 (norma $\ell_{1}$ entre DEEs estimadas com o critério de Sturges), DEE-vc-KL (divergência de Kullback-Leibler entre DEEs estimadas com validação cruzada não viesada), DEE-vc-l1 (norma $\ell_{1}$ entre DEEs estimadas com validação cruzada não viesada), DAA-KL (divergência de Kullback-Leibler entre DAAs), e DAA-l1 (norma $\ell_{1}$ entre DAAs). A linha vermelha tracejada indica o valor esperado. 
Observamos na Figura 5.2 que, quando $p_{s}=1.4$, os resultados obtidos utilizando o critério de Sturges e a validação cruzada não viesada se aproximam mais do valor esperado do que os obtidos pelo critério de Silverman. Além disso, o estimador apresentou menor variância com o critério de validação cruzada não viesada.

Para avaliar a diferença de desempenho entre os critérios de escolha de largura de banda em cenários diversos, aplicamos os estimadores de parâmetro a grafos BA gerados aleatoriamente com $p_{s}=1.1,1.3,1.5,1.7,1.9$ e $n=100,500$. Observamos, na Figura 5.3, que quando $p_{s}=1.1,1.3,1.5,1.7$ e $n=100$, o valor mediano continua sendo mais próximo do esperado utilizando o critério de Sturges e de validação cruzada não viesada ao invés do critério de Silverman.

Contudo, notamos nas Figuras 5.3b, 5.3d e 5.3e que, quando $p_{s}=1.3,1.7$ e 1.9, a variância é maior quando utilizamos o critério de Sturges. Se aumentarmos o tamanho do grafo para $n=500$, a variância obtida com o método baseado no critério de Sturges é maior em todos os cenários considerados $\left(p_{s}=1.1,1.3,1.5,1.7\right.$ e 1.9), como podemos ver na Figura 5.4. Além disso, observamos que, quando o grafo é suficientemente grande $(n=500)$, podemos obter resultados medianos mais próximos do valor esperado com o critério de Silverman quando comparado ao critério de Sturges, como vemos na Figura 5.4 para $p_{s}=1.7$ e 1.9. Já, o método baseado na validação cruzada não viesada apresentou resultados mais próximos ao esperado e menor variância para todos os valores de parâmetro considerados. Assim, nota-se que o critério para escolha da largura de banda utilizado na estimação da densidade espectral pode alterar o desempenho do estimador de parâmetro.

Alternativamente, podemos substituir a densidade espectral empírica pela distribuição acumulada dos autovalores (DAA). Na Figura 5.2, em que exibimos boxplots do estimador de parâmetro para grafos BA com $n=500$ e $p_{s}=1.4$, o boxplot DAA-KL corresponde aos resultados obtidos utilizando a divergência de Kullback-Leibler entre a DAA do grafo e do modelo. Observamos que nesse cenário o valor mediano obtido com o estimador baseado na distribuição acumulada dos autovalores se aproxima mais do parâmetro esperado e apresenta menor variância do que os estimadores baseados na densidade espectral utilizando os critérios de Silverman e Sturges. Já, comparado ao método baseado na validação cruzada não viesada, o estimador baseado na distribuição acumulada apresentou resultados similares.

Além da divergência de Kullback-Leibler, consideramos a norma $\ell_{1}$ (denotada por $\|\cdot\|_{1}$ ) entre as distribuições dos autovalores. Nesse caso, nós temos

$$
D\left(\mu^{\mathcal{G}}\left(\phi_{\cdot, \sigma}\right), \mu_{\theta}\left(\phi_{\cdot, \sigma}\right)\right)=\left\|\mu^{\mathcal{G}}\left(\phi_{\cdot, \sigma}\right)-\mu_{\theta}\left(\phi_{\cdot, \sigma}\right)\right\|_{1}=\int\left|\mu^{\mathcal{G}}\left(\phi_{x, \sigma}\right)-\mu_{\theta}\left(\phi_{x, \sigma}\right)\right| d x .
$$

Nas Figuras 5.2, 5.3 e 5.4 os boxplots DEE-11, DEE-St-11, DEE-vc-11 e DAA-11 correspondem aos resultados obtidos a partir da norma $\ell_{1}$ entre densidades espectrais empíricas estimadas com o critério de Silverman, densidades espectrais empíricas estimadas com o critério de Sturges, densidades espectrais empíricas estimadas por validação cruzada não viesada e distribuições acumuladas dos autovalores, respectivamente. Observamos que os resultados obtidos com a norma $\ell_{1}$ são semelhantes aos obtidos com a divergência de Kullback-Leibler. 
(a) $p_{s}=1.1$

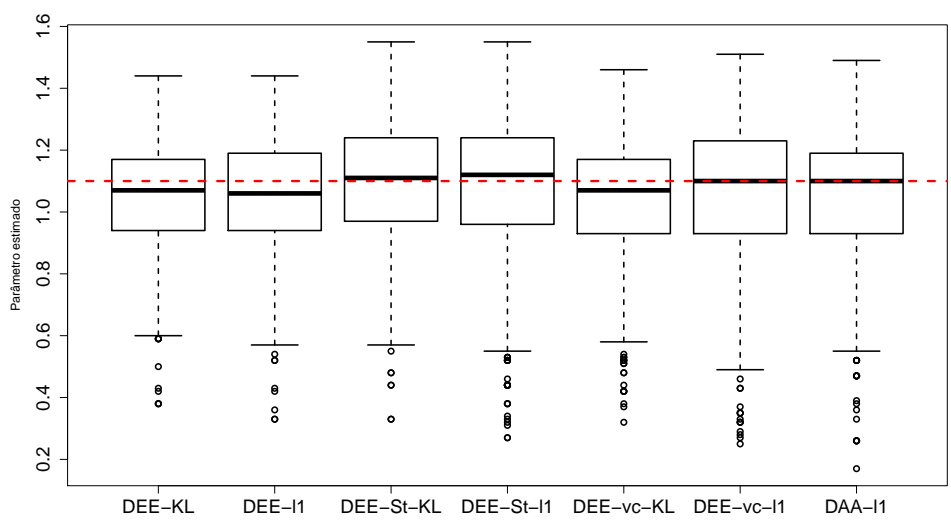

(c) $p_{s}=1.5$

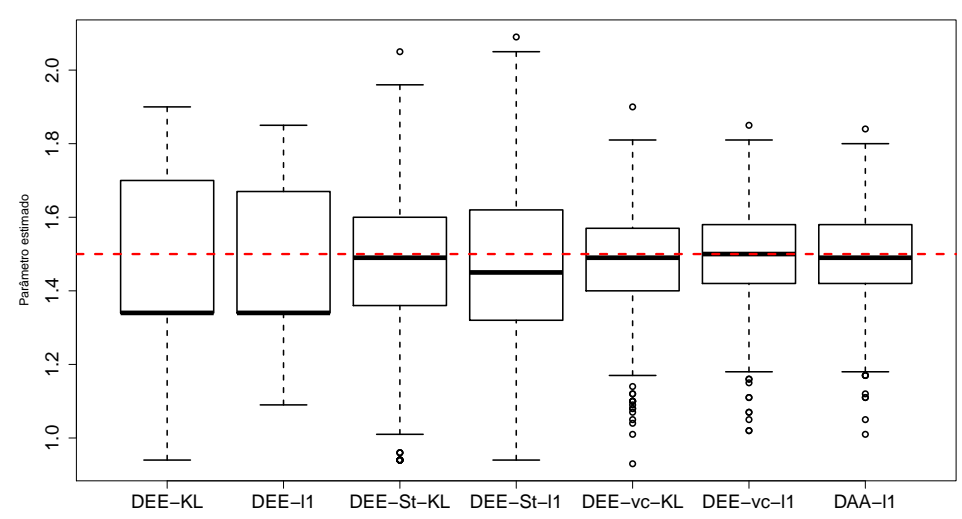

(b) $p_{s}=1.3$

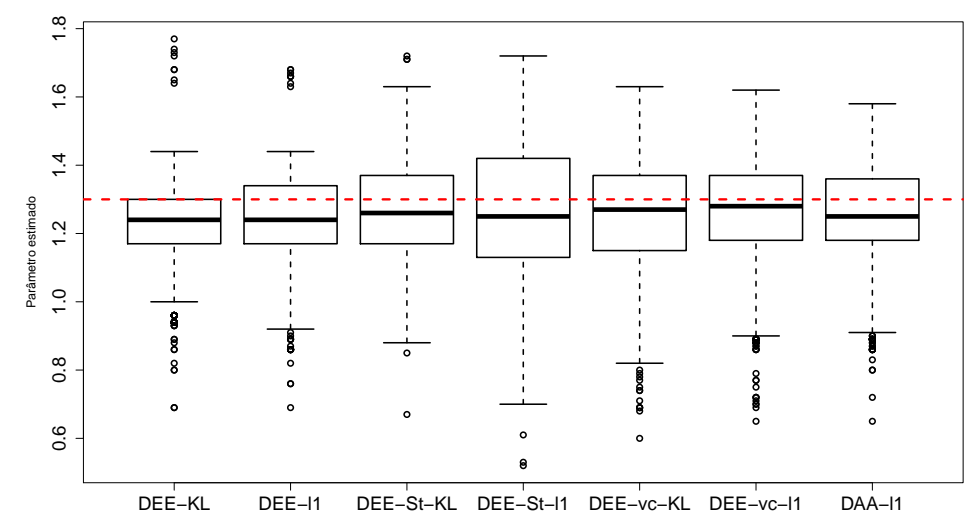

(d) $p_{s}=1.7$

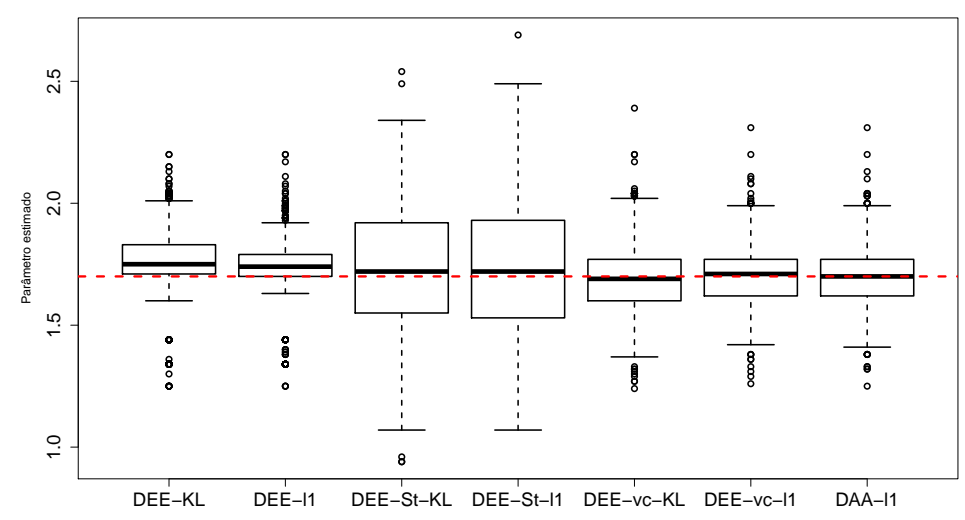

(e) $p_{s}=1.9$

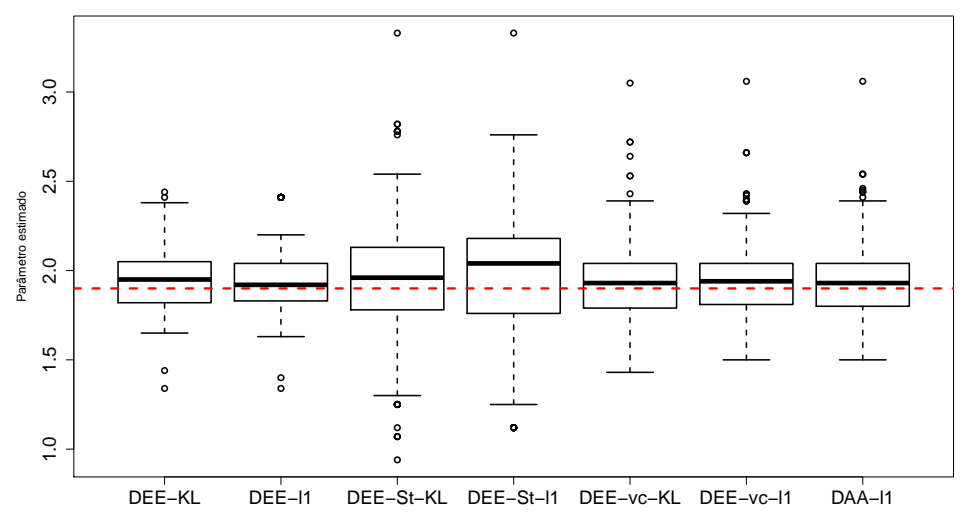

Figura 5.3: Boxplots dos estimadores de parâmetro para grafos BA de tamanho $n=100 \mathrm{com}$ expoente de escala $(a) p_{s}=1.1,(b) p_{s}=1.3,(c) p_{s}=1.5,(d) p_{s}=1.7$ e (e) $p_{s}=1.9$. Cada boxplot é nomeado pelo método utilizado para ajustar o modelo: DEE-KL (divergência de Kullback-Leibler entre DEEs estimadas com o critério de Silverman), DEE-l1 (norma $\ell_{1}$ entre DEEs estimadas com o critério de Silverman), DEE-St-KL (divergência de Kullback-Leibler entre DEEs estimadas com o critério de Sturges), DEE-St-l1 (norma $\ell_{1}$ entre DEEs estimadas com o critério de Sturges), DEE-vc$K L$ (divergência de Kullback-Leibler entre DEEs estimadas com validação cruzada não viesada), DEE-vc-l1 (norma $\ell_{1}$ entre DEEs estimadas com validação cruzada não viesada) e DAA-l1 (norma $\ell_{1}$ entre DAAs). A linha vermelha tracejada indica o valor esperado. 
(a) $p_{s}=1.1$

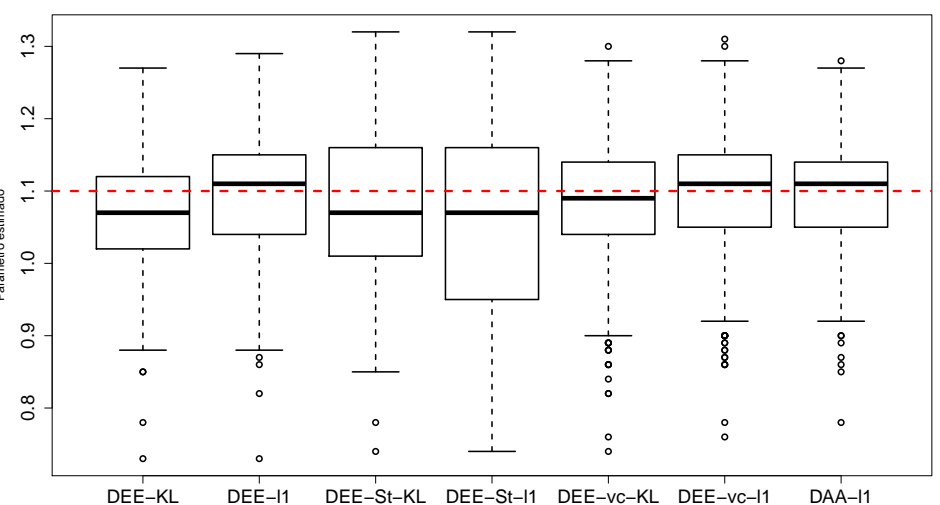

(c) $p_{s}=1.5$

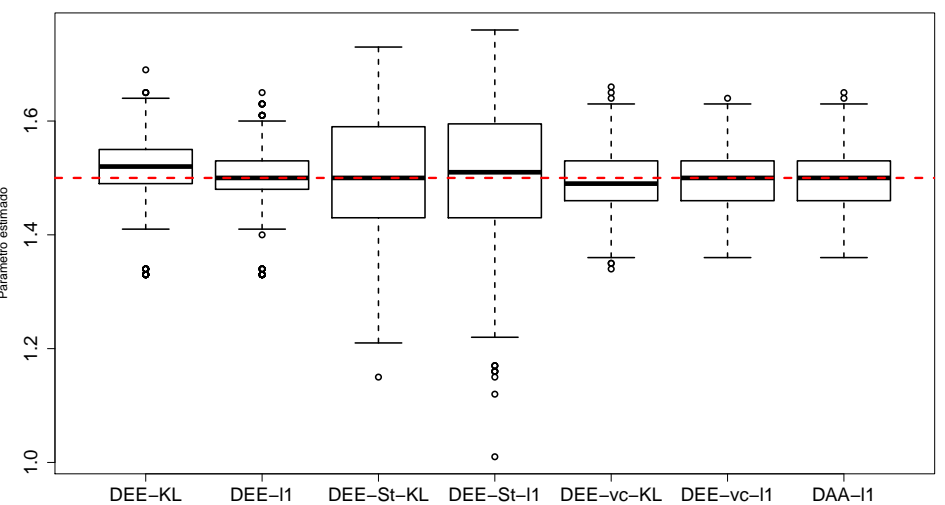

(b) $p_{s}=1.3$

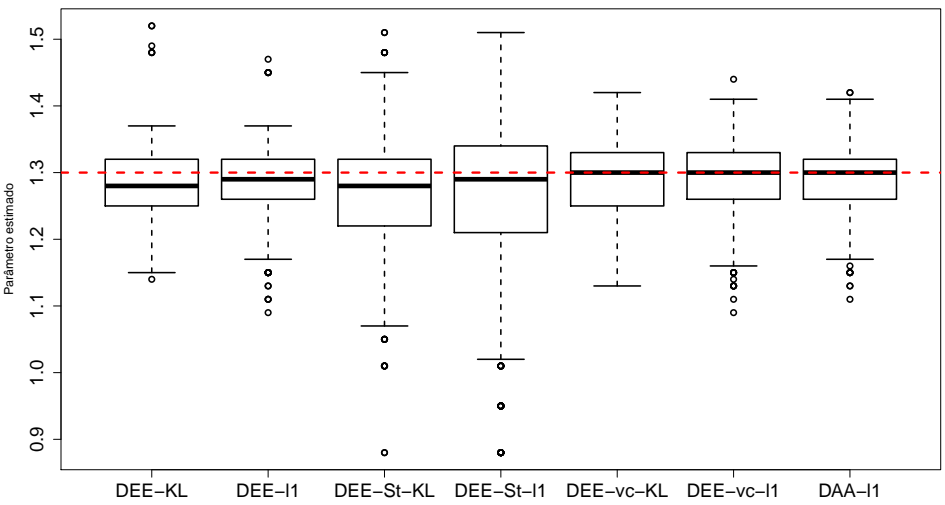

(d) $p_{s}=1.7$

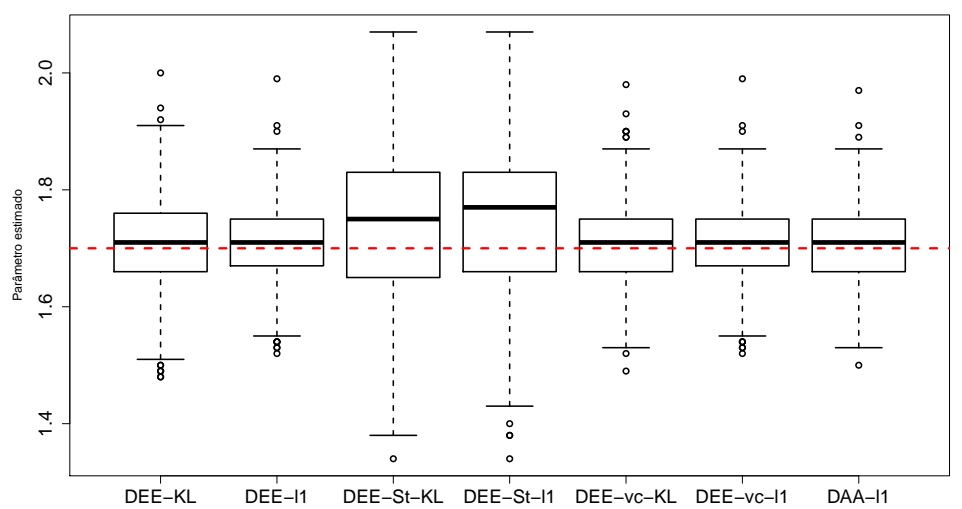

(e) $p_{s}=1.9$

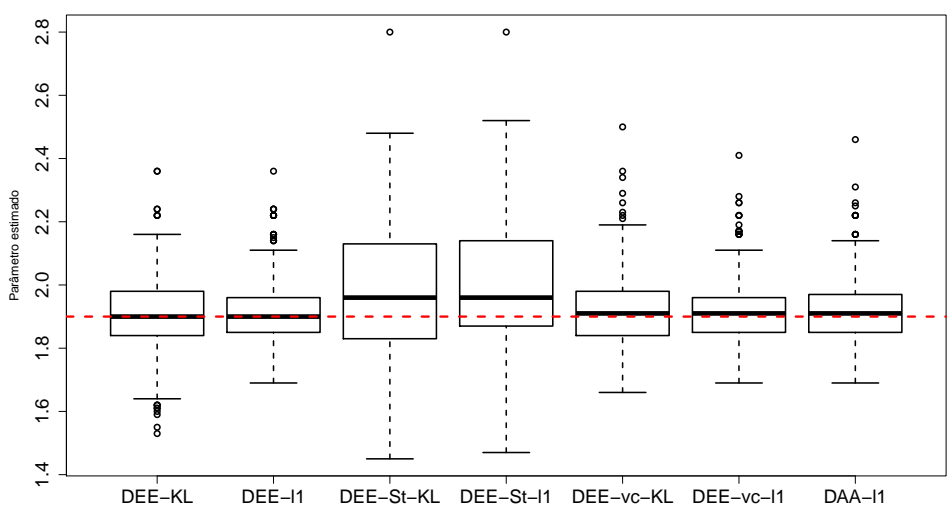

Figura 5.4: Boxplots dos estimadores de parâmetro para grafos BA de tamanho $n=500 \mathrm{com}$ expoente de escala $(a) p_{s}=1.1,(b) p_{s}=1.3$, (c) $p_{s}=1.5,(d) p_{s}=1.7$ e (e) $p_{s}=1.9$. Cada boxplot é nomeado pelo método utilizado para ajustar o modelo: DEE-KL (divergência de Kullback-Leibler entre DEEs estimadas com o critério de Silverman), DEE-l1 (norma $\ell_{1}$ entre DEEs estimadas com o critério de Silverman), DEE-St-KL (divergência de Kullback-Leibler entre DEEs estimadas com o critério de Sturges), DEE-St-l1 (norma $\ell_{1}$ entre DEEs estimadas com o critério de Sturges), DEE-vcKL (divergência de Kullback-Leibler entre DEEs estimadas com validação cruzada não viesada), DEE-vc-l1 (norma $\ell_{1}$ entre DEEs estimadas com validação cruzada não viesada) e DAA-l1 (norma $\ell_{1}$ entre DAAs). A linha vermelha tracejada indica o valor esperado. 
Ao utilizarmos a norma $\ell_{1}$, observamos que, de forma geral, os resultados obtidos com as distribuições acumuladas foram semelhantes aos obtidos com as DEEs estimadas por validação cruzada não viesada. Em comparação aos métodos baseados nos critérios de Silverman e Sturges, para grafos BA de tamanho $n=100$, os valores medianos dos estimadores obtidos pela distribuição acumulada e pela DEE estimada por validação cruzada não viesada se aproximam mais do parâmetro verdadeiro quando $p_{s}=1.5$ e ficam semelhantes quando $p_{s}=1.1,1.3,1.7,1.9$ (Figura 5.3). Para grafos de tamanho $n=500$ (Figura 5.4), em todos cenários considerados, a variância dos estimadores baseados na DAA e na DEE estimada por validação cruzada não viesada foi menor do que a do estimador baseado na DEE estimada com o critério de Sturges e semelhante à do estimador baseado no critério de Silverman. Além disso, quando $n=500$, os resultados a partir das distribuições acumuladas são tão ou mais próximos do valor esperado do que os resultados utilizando as DEEs com largura de banda escolhida pelo critério de Silverman e Sturges e semelhantes aos obtidos com DEEs estimadas por validação cruzada não viesada.

Adicionalmente ao modelo BA, observamos os resultados obtidos para os modelos ER $(p=0.5)$, DR $(d=5)$, GE $(r=0.5)$ e WS $\left(p_{r}=0.5\right)$, a partir de 1000 grafos de tamanho $n=100$ (Figura 5.5). Nesses cenários, todas as variantes do estimador de parâmetros apresentaram resultado similar.

Para o modelo $\mathrm{BE}$, geramos 1000 grafos com $n=600$ vértices, dois grupos de mesmo tamanho, probabilidade intergrupo de $0.2\left(p_{0}\right)$ e probabilidade intragrupo de $0.7\left(p_{1}\right)$. Estimamos os parâmetros $p_{0}$ e $p_{1}$ simultaneamente e obtivemos os resultados exibidos na Figura 5.6. Cada gráfico exibe o bagplot (boxplot bidimensional) obtido com cada um dos métodos (DEE-KL, DEE-11, DEE-St-KL, DEE-St-11, DEE-vc-KL, DEE-vc-11 e DAA11). As linhas verdes tracejadas na vertical e horizontal mostram, respectivamente, os valores esperados para $p_{0}$ e $p_{1}$. O asterisco em vermelho indica a mediana. Observamos que todos os estimadores se aproximaram do valor esperado, isto é, o asterisco vermelho se aproximou da intersecção entre as duas retas verdes. Além disso, notamos que os métodos que utilizam validação cruzada, o critério de Silverman e os baseados na divergência de KL entre densidades estimadas pelo critério de Sturges apresentaram menor variância.

Assim, em todos os cenários considerados, o estimador se aproximou dos valores esperados utilizando as adaptações propostas, como a norma $\ell_{1}$ e a distribuição acumulada dos autovalores. Uma das vantagens de se utilizar a norma $\ell_{1}$ ao invés da divergência de Kullback-Leibler é a sua relação com a definição de convergência fraca, que nos levou à derivação de propriedades teóricas do estimador de parâmetro, como mostramos na Seção 5.4. A distribuição acumulada, por sua vez, é estimada mais eficientemente $(O(n))$ do que a DEE baseada em validação cruzada não viesada $\left(O\left(n^{2}\right)\right)$ e sua utilização para estimação de parâmetros apresentou melhor desempenho em alguns cenários em comparação aos métodos baseados em DEEs estimadas pelo critério de Sturges ou Silverman. 
(a) $E R$

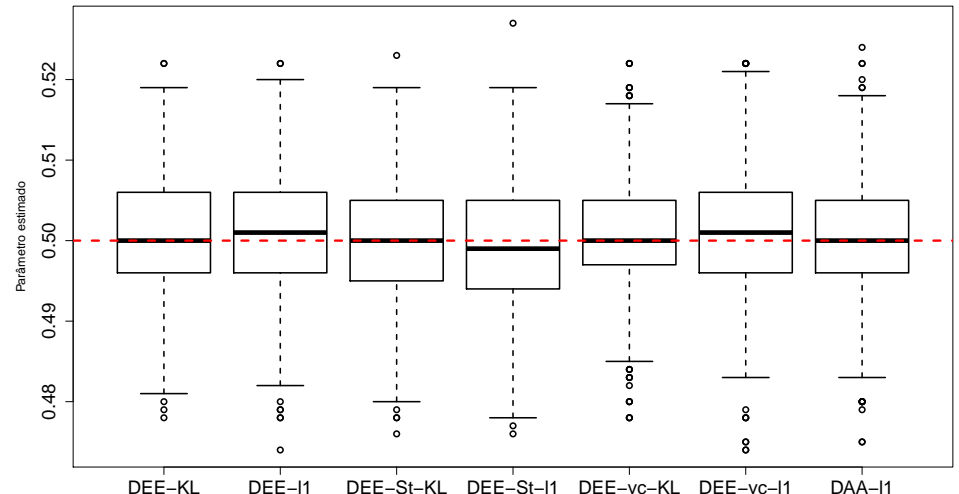

(c) $G E$

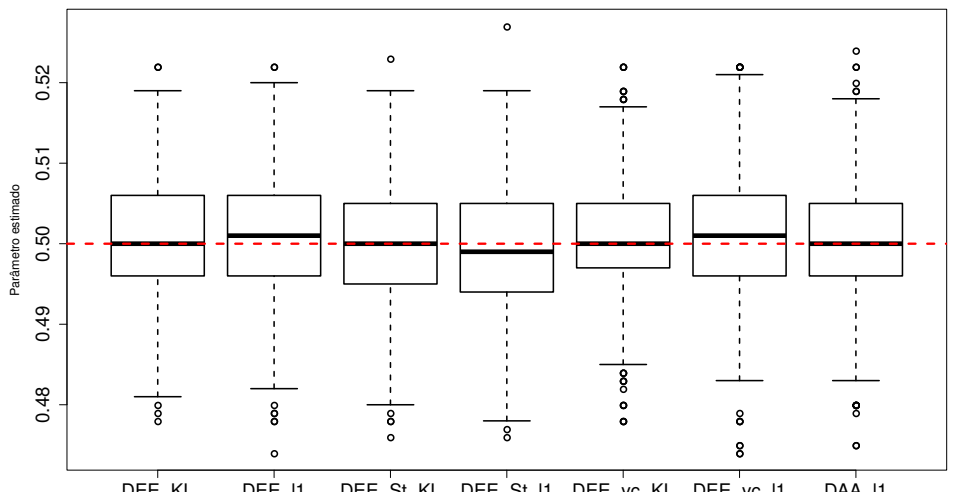

DEE-KL DEE-11 DEE-St-KL DEE-St-11 DEE-vc-KL DEE-vc-I1 DAA-11 (b) $D R$

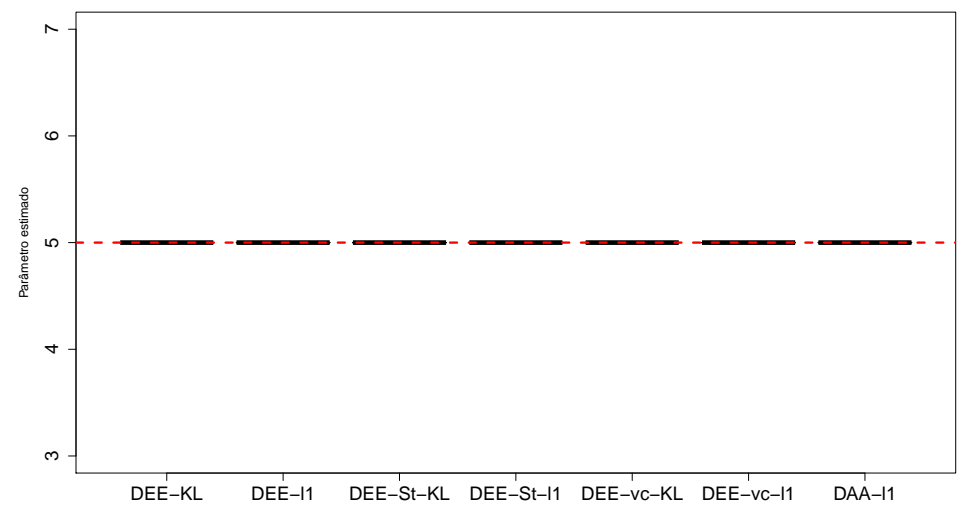

(d) $W S$

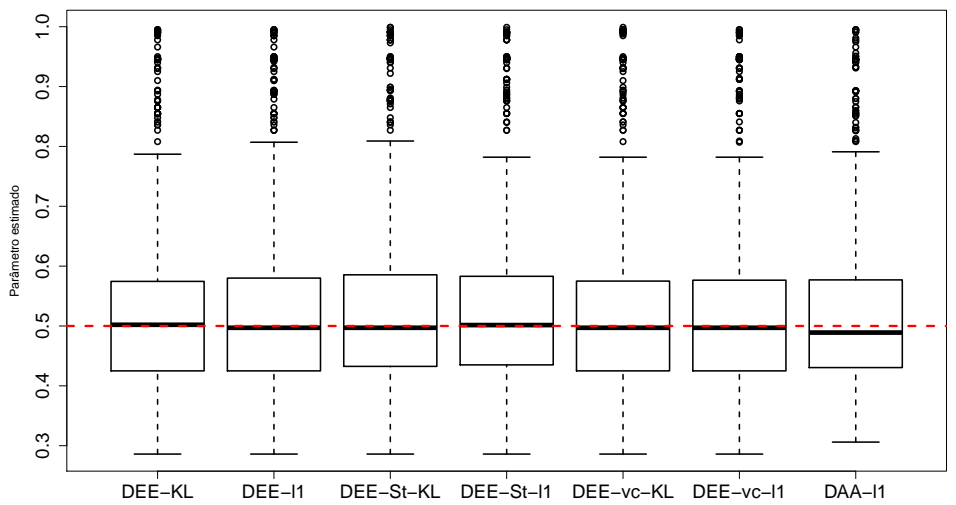

Figura 5.5: Boxplots dos estimadores de parâmetro para grafos gerados pelos modelos (a) ER, (b) $D R,(c) G E$ e (d) WS com tamanho $n=100$ e parâmetros $p=0.5$ (probabilidade de conectar cada par de vértices do ER), $d=5$ (grau do $D R$ ), $r=0.5$ (raio do GE) e $p_{r}=0.5$ (probabilidade de reconectar arestas do WS). Cada boxplot é nomeado pelo método utilizado para ajustar o modelo: DEE-KL (divergência de Kullback-Leibler entre DEEs estimadas com o critério de Silverman), DEE-l1 (norma $\ell_{1}$ entre DEEs estimadas com o critério de Silverman), DEE-St-KL (divergência de Kullback-Leibler entre DEEs estimadas com o critério de Sturges), DEE-St-l1 (norma $\ell_{1}$ entre DEEs estimadas com o critério de Sturges), DEE-vc-KL (divergência de Kullback-Leibler entre DEEs estimadas com validação cruzada não viesada), DEE-vc-l1 (norma $\ell_{1}$ entre DEEs estimadas com validação cruzada não viesada) e DAA-l1 (norma $\ell_{1}$ entre DAAs). A linha vermelha tracejada indica o valor esperado. 
(a) $D E E-K L$

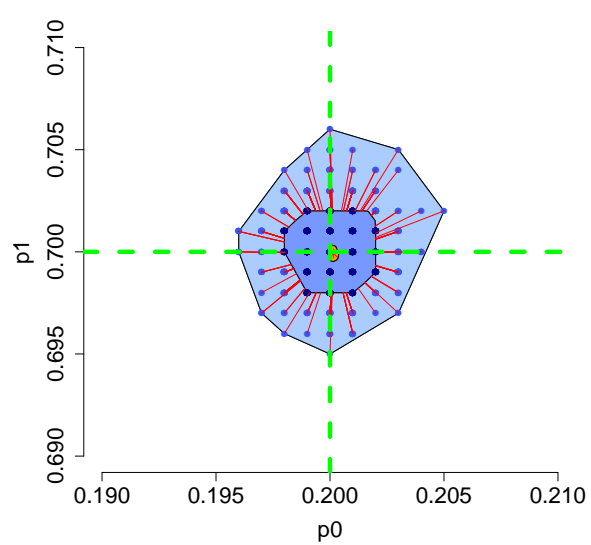

(d) $D E E-S t-l 1$

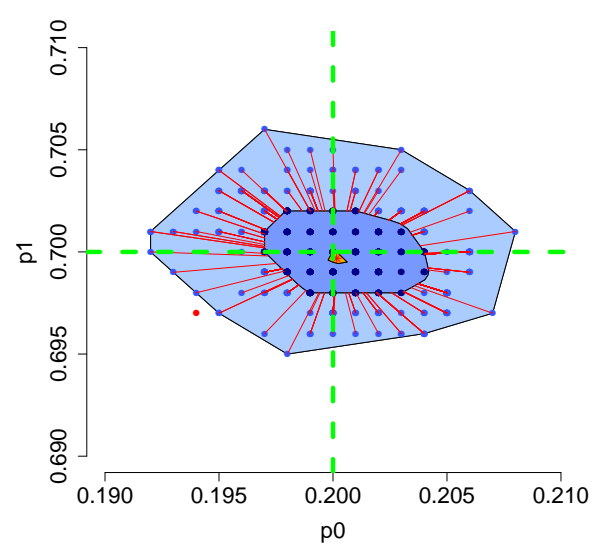

(b) $D E E-l 1$

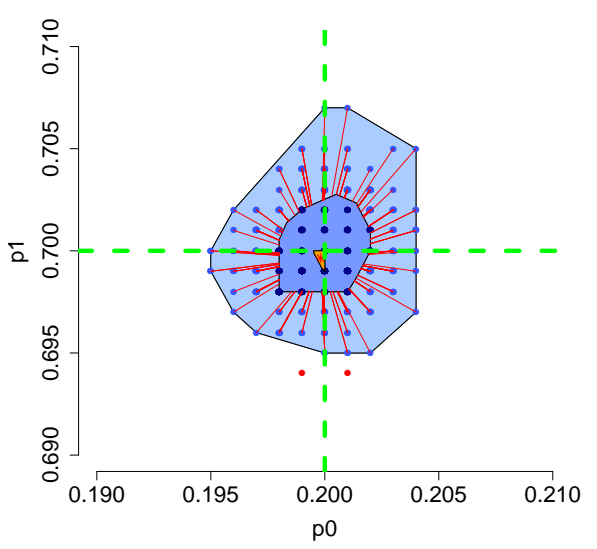

(e) $D E E-v c-K L$

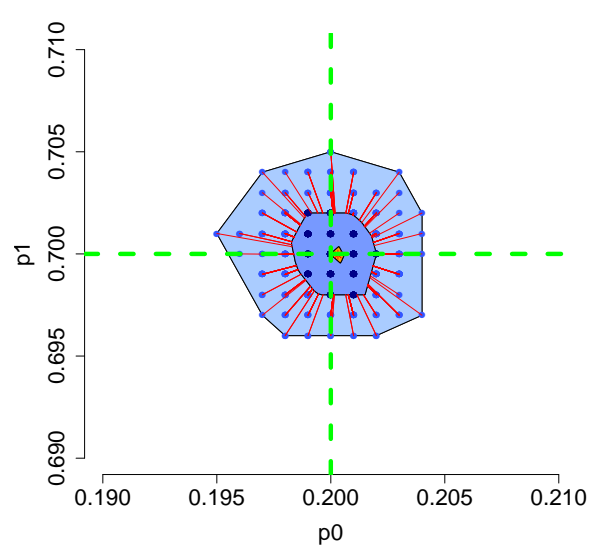

(g) $D A A-l 1$

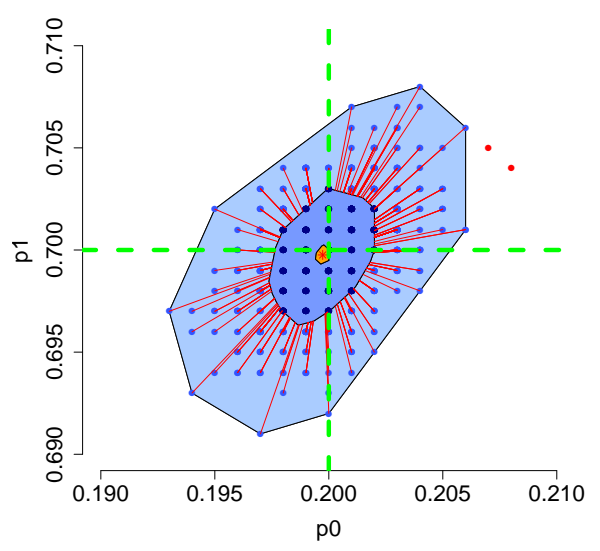

(c) $D E E-S t-K L$

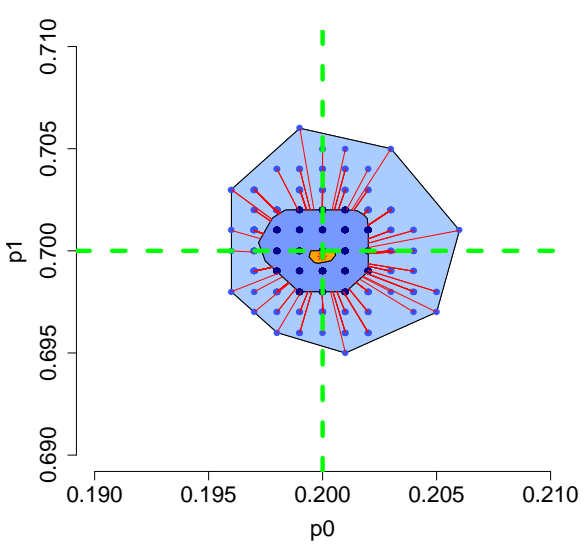

(f) $D E E-v c-l 1$

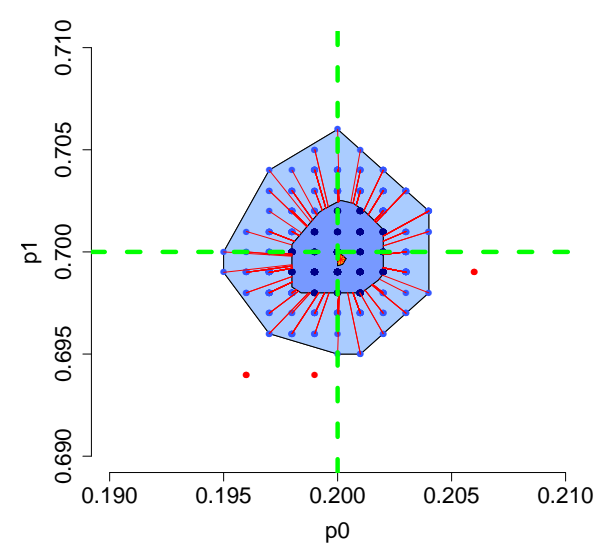

Figura 5.6: Bagplots dos estimadores de parâmetro para grafos gerados pelos modelo BE baseados na (a) divergência KL entre DEEs estimadas com o critério de Silverman (DEE-KL), (b) norma $\ell_{1}$ entre DEEs estimadas com o critério de Silverman (DEE-l1), (c) divergência de Kullback-Leibler entre DEEs estimadas com o critério de Sturges (DEE-St-KL), (d) norma $\ell_{1}$ entre DEEs estimadas com o critério de Sturges (DEE-St-l1), (e) divergência de Kullback-Leibler entre DEEs estimadas com validação cruzada não viesada (DEE-vc-KL), $(f)$ norma $\ell_{1}$ entre DEEs estimadas com validação cruzada não viesada (DEE-vc-l1) e (g) norma $\ell_{1}$ entre DAAs (DAA-l1). Foram gerados 1000 grafos com 600 vértices, dois blocos de mesmo tamanho, probabilidade intergrupo $p_{0}=0.2$ e probabilidade intragrupo $p_{1}=\quad$ 0.7. Em cada gráfico, os eixo x e y mostram os valores estimados para $p_{0}$ e $p_{1}$, respectivamente. $O$ asterisco em vermelho indica o ponto mediano estimado. $O$ polígono azul interior destaca metade dos pontos ao redor da mediana. O polígono azul maior, tem três vezes o tamanho do polígono interior. Pontos fora do polígono maior são outliers. Linhas tracejadas verdes indicam o valor esperado para cada parâmetro. Assim, espera-se que os pontos estejam próximos à intersecção das duas linhas verdes. 


\subsection{Propriedades de convergência}

Para estudar o comportamento dos estimadores de parâmetro baseados na norma $\ell_{1}$, utilizamos resultados de DACUNHA-CASTELle e Duflo, 1986 sobre estimadores de mínimo contraste, detalhados na Seção 5.4.1. Em seguida, na Seção 5.4.2, com base no Teorema 3.2.8 de Dacunha-Castelle e Duflo, 1986, derivamos a consistência dos estimadores propostos e apresentamos os Teoremas 2 e 3 sobre os métodos baseados na DEE e DAA, respectivamente.

\subsubsection{Estimadores de mínimo contraste}

Dacunha-Castelle e Duflo, 1986 definem um estimador de mínimo contraste como a seguir.

Definição 1. Considere um modelo estatístico $\left(\Omega, A,\left(P_{\theta}\right)_{\theta \in \Theta}\right)$. Nós chamamos de função de contraste desse modelo relativa a $\theta$, a função $\theta \mapsto D\left(\theta, \theta^{\star}\right)$ de $\Theta$ em $\mathbb{R}_{+}$com mínimo estrito em $\theta=\theta^{\star}$. Se os experimentos são descritos por um filtro $\mathbb{F}=\left(F_{n}\right)_{n \in T}\left(T=\mathbb{N}\right.$ ou $\left.T=\mathbb{R}_{+}\right)$, um contraste (ou processo de contraste) relativo a $\theta^{\star}$ e a $D$ é uma função $U$ (independente de $\theta^{\star}$ ) de $\Theta \times T \times \Omega$ em $\mathbb{R}$, denotada por $(\theta, n, \omega) \mapsto U_{n}(\theta, \omega)$, satisfazendo as seguintes propriedades:

1. Para cada $(\theta, n) \in \Theta \times T$, a variável aleatória $U_{n}(\theta): \omega \mapsto U_{n}(\theta, \omega)$ é $F_{n}$-mensurável: $\left(U_{n}(\theta)\right)$ é um processo $\mathbb{F}$-adaptado.

2. $\left(U_{n}(\theta)\right)$ converge em probabilidade (com respeito a $\left.P_{\theta^{\star}}\right)$ para $D\left(\theta, \theta^{\star}\right)$ à medida que $n \rightarrow \infty$.

Finalmente, um estimador de contraste mínimo associado a $U$ é um estimador $\mathbb{F}$-adaptado $\left(\hat{\theta}_{n}\right)_{n \in T}$ tal que, para cada n, nós temos:

$$
U_{n}\left(\hat{\theta}_{n}\right)=\inf \left\{U_{n}(\theta) ; \theta \in \Theta\right\}
$$

Usando as definições anteriores, temos o resultado a seguir.

Teorema (3.2.8 de Dacunha-CAstelle e Duflo, 1986). Suponha que $\theta^{\star}$ é o valor verdadeiro (desconhecido) do parâmetro. Considere as seguintes hipóteses:

1. $\Theta$ é um conjunto compacto do $\mathbb{R}^{k}$.

2. Os mapas $\theta \mapsto D\left(\theta, \theta^{\star}\right)$ e $\theta \mapsto U_{n}(\theta, \omega)$, para cada $\omega$, são contínuos.

3. Denotando, para cada $\eta>0$,

$$
w(n, \eta)=\sup \left\{\left|U_{n}(\theta)-U_{n}(\beta)\right| ;|\theta-\beta| \leq \eta\right\},
$$

existem duas sequências $\left(\eta_{k}\right)$ e $\left(\epsilon_{k}\right)$, ambas decrescendo para 0, tais que, para cada $k$,

$$
P\left[w\left(n, \eta_{k}\right)>\epsilon_{k}\right] \underset{n \rightarrow \infty}{\longrightarrow} 0 .
$$

Então, cada estimador de mínimo contraste $\left(\hat{\theta}_{n}\right)$ é consistente em $\theta$. 
Com base na Definição 1, mostramos na seção a seguir que o estimador (5.1), com $D$ sendo a norma $\ell_{1}$, é de mínimo contraste quando conhecemos a função de densidade analiticamente. Temos $T=\mathbb{N}$, uma vez que cada unidade de tempo corresponde a um tamanho de grafo.

\subsubsection{Estimadores baseados na norma $\ell_{1}$ entre distribuições de autovalores}

Nesta Seção consideramos $D$ como sendo a norma $\ell_{1}$. Representamos por $\mathcal{M}_{1}(\Lambda)$ o conjunto de medidas de probabilidade sobre o espectro $\Lambda$. Se assumirmos que a densidade espectral do modelo de grafo aleatório é conhecida analiticamente, o estimador baseado na norma $\ell_{1}$ entre de DEEs é consistente, conforme provamos no Teorema 2.

Teorema 2. Seja $\left\{P_{\theta} ; \theta \in \Theta\right\}$ um modelo de grafo aleatório parametrizado e assuma que, para todo $\theta \in \Theta$, existe a medida de probabilidade dos autovalores assintótica $\mu_{\theta}$ (com respeito à convergência fraca quase certa). Nós assumimos que $\mu_{\theta} \in \mathcal{M}_{1}(\Lambda)$, onde $\Lambda$ é um conjunto limitado. Considere a sequência de grafos aleatórios $\left(\mathcal{G}_{n}\right)_{n \geq 1}$, da distribuição $P_{\theta^{\star}}$. Seja $\phi$ um kernel e $\sigma=\sigma_{n}$ a largura de banda (bandwidth) que converge para zero. Se o mapa $\theta \in$ $\Theta \mapsto \mu_{\theta}(d \lambda) \in \mathcal{M}_{1}(\Lambda)$ é injetor, contínuo e $\Theta$ é compacto, então o minimizador

$$
\hat{\theta}_{n}=\underset{\theta}{\operatorname{argmin}}\left\|\mu^{\mathcal{G}_{n}}\left(\phi_{\cdot, \sigma}\right)-\mu_{\theta}\left(\phi_{\cdot, \sigma}\right)\right\|_{1}
$$

converge em probabilidade para $\theta^{\star}$ à medida que $n \rightarrow \infty$.

Demonstração. A prova é baseada no Teorema 3.2.8 de Dacunha-Castelle e Duflo, 1986 sobre estimadores de mínimo contraste. Denotamos o contraste $U_{n}\left(\theta, \mathcal{G}_{n}\right)$ por $D_{n}\left(\theta, \theta^{\star}\right)$. Considerando a norma $\ell_{1}$ entre densidades como a função de contraste, definimos $D_{n}\left(\theta, \theta^{\star}\right):=\left\|\mu^{\mathcal{C}_{n}}\left(\phi_{\cdot, \sigma}\right)-\mu_{\theta}\left(\phi_{\cdot, \sigma}\right)\right\|_{1}$.

O primeiro passo da prova é estabelecer a convergência do contraste $D_{n}\left(\theta, \theta^{\star}\right)$. Supondo que $\mu_{\theta}$ existe para todo $\theta \in \Theta$, nós temos que para cada $x \in \mathbb{R}$,

$$
\mu^{\mathcal{G}_{n}}\left(\phi_{x, \sigma}\right)=\frac{1}{n} \sum_{i=1}^{n} \phi_{x, \sigma}\left(\lambda_{i}^{\mathcal{G}_{n}} / \sqrt{n}\right) \underset{n \rightarrow \infty}{\longrightarrow} \mu_{\theta^{\star}}\left(\phi_{x, \sigma}\right)
$$

$P_{\theta^{\star}}$-quase certamente. Então, pelo Teorema da convergência dominada, nós temos

$$
D_{n}\left(\theta, \theta^{\star}\right) \underset{n \rightarrow \infty}{\longrightarrow}\left\|\mu_{\theta^{\star}}\left(\phi_{;, \sigma}\right)-\mu_{\theta}\left(\phi_{;, \sigma}\right)\right\|_{1}:=D\left(\theta, \theta^{\star}\right),
$$

$P_{\theta^{\star}}$ quase certamente. Agora, o segundo passo é estabelecer que a função assintótica $\theta \mapsto D\left(\theta, \theta^{\star}\right)$ tem um mínimo estrito em $\theta^{\star}$. Note que o limite $D\left(\theta, \theta^{\star}\right)$ é mínimo se, e somente se, $\mu_{\theta^{\star}}(\phi)=\mu_{\theta}(\phi)$ quase certamente (com respeito à medida de Lebesgue), isto é,

$$
\forall x \in \mathbb{R}, \quad \int \phi[(x-\lambda) / \sigma] \mu_{\theta^{*}}(d \lambda)=\int \phi[(x-\lambda) / \sigma] \mu_{\theta}(d \lambda) .
$$


Propriedades clássicas de kernels implicam que quando $\sigma_{n} \rightarrow 0$, nós temos

$$
\mu_{\theta}(d \lambda)=\mu_{\theta^{\star}}(d \lambda)
$$

Como $\theta \mapsto \mu_{\theta}$ é injetora, isso implica que $\theta=\theta^{\star}$. Assim, o limite $D\left(\theta, \theta^{\star}\right)$ é mínimo se, e somente se, $\theta=\theta^{\star}$.

Finalmente, o mapa $\theta \mapsto \mu_{\theta}(d \lambda)$ é contínuo no conjunto compacto $\Theta$ e assim uniformemente contínuo. Isso implica que

$$
\forall \epsilon>0, \exists \eta>0 \text { tal que se }\left\|\theta-\theta^{\prime}\right\| \leq \eta \text { então }\left|\mu_{\theta}(d \lambda)-\mu_{\theta^{\prime}}(d \lambda)\right| \leq \epsilon .
$$

Assim, nós temos que

$$
\begin{aligned}
\left|D_{n}\left(\theta, \theta^{\star}\right)-D_{n}\left(\theta^{\prime}, \theta^{\star}\right)\right| & \leq\left\|\mu_{\theta}\left(\phi_{, \sigma \sigma}\right)-\mu_{\theta^{\prime}}\left(\phi_{, \sigma}\right)\right\|_{1} \\
& \leq \sigma^{-1} \iint_{\Lambda} \phi[(x-\lambda) / \sigma] \times\left|\mu_{\theta}(d \lambda)-\mu_{\theta^{\prime}}(d \lambda)\right| d x \\
& \leq \iint \phi(y) \times\left|\mu_{\theta}(x-\sigma y)-\mu_{\theta^{\prime}}(x-\sigma y)\right| d y d x \\
& \leq \epsilon|\Lambda|,
\end{aligned}
$$

onde $|\Lambda|$ denota o comprimento do intervalo.

Por uma questão de clareza, nós assumimos na última linha que $\mu_{\theta}$ é uma medida com uma densidade, mas isso não tem consequências uma vez que $\left\|\theta-\theta^{\prime}\right\| \leq \eta$. Isso estabelece uma convergência uniforme (com respeito a $\theta$ ) da função de contraste $D_{n}$ à medida que $n$ tende para infinito. Aplicando o Teorema 3.2.8 de Dacunha-CAstelle e Duflo, 1986, nós obtemos o resultado desejado.

Um resultado similar pode ser estabelecido quando consideramos funções de distribuição acumulada dos autovalores no lugar de densidades espectrais, como mostramos a seguir.

Teorema 3. Seja $\left\{P_{\theta} ; \theta \in \Theta\right\}$ um modelo de grafo aleatório parametrizado e assuma que, para todo $\theta \in \Theta$, existe a medida de probabilidade dos autovalores assintótica $\mu_{\theta}$ (com respeito à convergência fraca quase certa). Nós assumimos que $\mu_{\theta} \in \mathcal{M}_{1}(\Lambda)$, onde $\Lambda$ é um conjunto limitado. Considere a sequência de grafos aleatórios $\left(\mathcal{G}_{n}\right)_{n \geq 1}$, da distribuição $P_{\theta^{\star}}$. Se o mapa $\theta \in \Theta \mapsto \mu_{\theta}(d \lambda) \in \mathcal{M}_{1}(\Lambda)$ é injetor e contínuo e $\Theta$ é compacto, então o minimizador

$$
\hat{\theta}_{n}=\underset{\theta}{\operatorname{argmin}}\left\|F^{\mathcal{G}_{n}}-F_{\theta}\right\|_{1}
$$

converge em probabilidade para $\theta^{\star}$ à medida que $n \rightarrow \infty$.

Demonstração. A prova segue as mesmas linhas da prova anterior, mas primeiro notando que para qualquer $x$, a função $\lambda \mapsto \mathbb{1}\{\lambda \leq x\}$ é contínua em quase todo ponto. Assim

$$
F^{\mathcal{G}_{n}}(x) \underset{n \rightarrow \infty}{\longrightarrow} F_{\theta}(x)
$$


$P_{\theta^{\star}}$-quase certamente. Para o segundo passo, note que $F_{\theta}$ caracteriza inteiramente a distribuição $\mu_{\theta}$. Finalmente, nós provamos que

$$
\begin{aligned}
\left|D_{n}\left(\theta, \theta^{\star}\right)-D_{n}\left(\theta^{\prime}, \theta^{\star}\right)\right| & \leq\left\|F_{\theta}-F_{\theta^{\prime}}\right\|_{1} \\
& \leq \iint 1\{\lambda \leq x\}\left|\mu_{\theta}(d \lambda)-\mu_{\theta^{\prime}}(d \lambda)\right| d x \\
& \leq \epsilon|\Lambda|^{2},
\end{aligned}
$$

sempre que $\left\|\theta-\theta^{\prime}\right\| \leq \eta$.

\subsubsection{Simulações}

A seguir, nós descrevemos experimentos para avaliar o desempenho dos estimadores de parâmetro baseados na norma $\ell_{1}$ entre as densidades espectrais e entre as distribuições acumuladas dos autovalores. Utilizamos o kernel Gaussiano para estimar a DEE e o critério de Silverman para escolher a largura de banda (Silverman, 1986).

Primeiramente, nós ilustramos cenários nos quais as condições dos Teoremas 2 e 3 são válidas e uma forma analítica para a distribuição espectral assintótica é conhecida. Depois, nós exploramos cenários nos quais as suposições podem não ser válidas e/ou não conhecemos a distribuição assintótica.

\section{Ilustração dos Teoremas}

Para ilustrar os Teoremas 2 e 3, nós consideramos cenários nos quais uma forma analítica para a DEE é conhecida, como nos modelos ER e DR. No modelo de ER, espera-se que DEE de $A / \sqrt{n}$ convirja para a lei semi-circular quando $p=\omega\left(n^{-1}\right)$. Nesse caso, as suposições dos Teoremas 2 e 3 valem quando $\Theta=[0,0.5]$ ou $\Theta=[0.5,1]$ e o minimizador da norma $\ell_{1}$ para o problema (5.1) converge em probabilidade para o parâmetro verdadeiro. Nós geramos 1000 grafos de tamanhos $n=20,50,100,500,1000,10000$ com parâmetros $p=0.1,0.3,0.5,0.7,0.9$. Na Figura 5.7, nós mostramos o parâmetro ajustado médio com um intervalo de confiança de $95 \%$ para o modelo ER usando a densidade espectral (linha azul) e a distribuição acumulada (linha verde tracejada). O parâmetro verdadeiro é indicado pela linha vermelha pontilhada.

Similarmente, nós realizamos experimentos de simulação com o grafo aleatório $d$ regular no qual a DEE de $\mathrm{A} / \sqrt{d-1}$ converge fracamente quase certamente para a lei de Kesten-McKay. Nós geramos 1000 grafos de tamanhos $n=20,50,100,500,1000,10000$ com parâmetros $d=3,5,10, \sqrt{n}$. Na Figura 5.8 , nós mostramos o parâmetro ajustado médio com um intervalo de confiança de $95 \%$ para o modelo DR usando a densidade espectral (linha azul) e a distribuição acumulada (linha verde tracejada). O valor esperado é indicado pela linha vermelha pontilhada.

Como esperado pelos Teoremas 2 e 3, nós observamos nas Figuras 5.7 e 5.8 que o parâmetro ajustado médio se aproxima do parâmetro verdadeiro à medida que o número de vértices aumenta. No modelo ER, o estimador baseado em DEE se aproximou mais rapidamente do valor desejado para $p=0.1,0.7,0.9$. Já no modelo $\mathrm{DR}$, o método baseado em DAA apresentou teve resultado superior para $d=5$ e inferior para $d=3$. 
(a) $p=0.1$

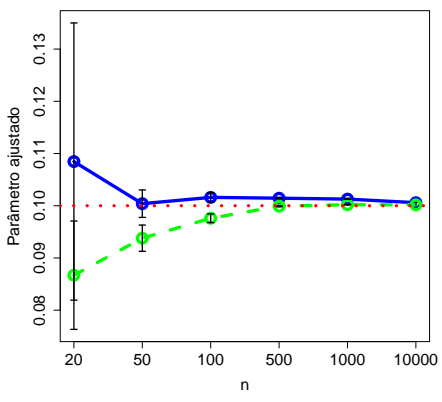

(d) $p=0.7$

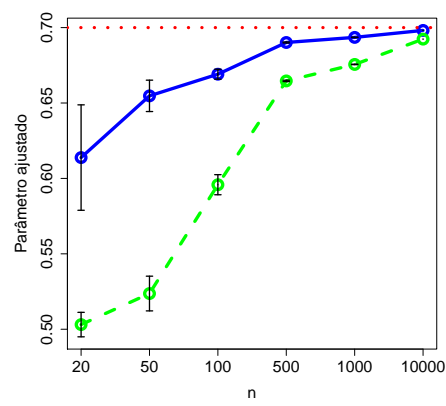

(b) $p=0.3$

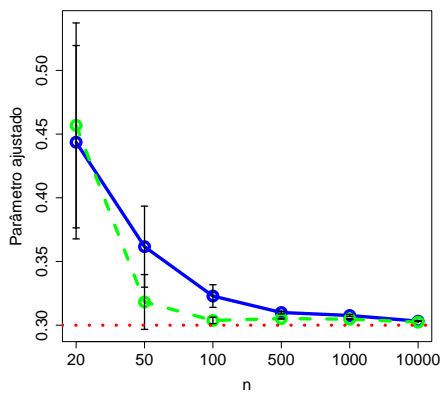

(e) $p=0.9$

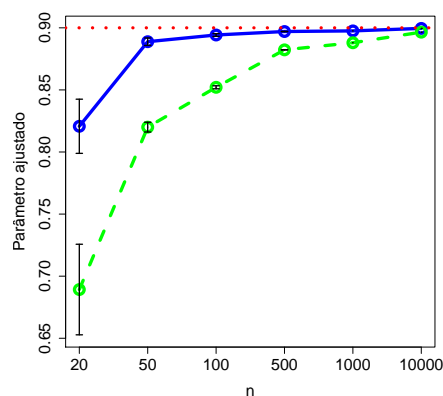

(c) $p=0.5$

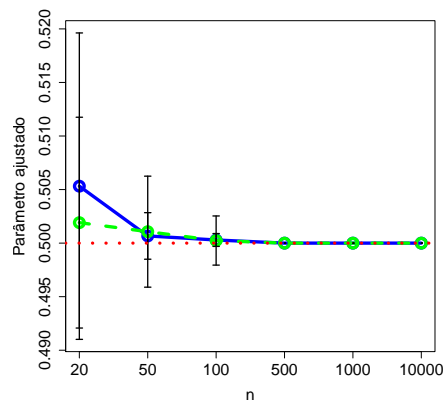

- Média DEE

- Média DAA

... Valor esperado

Figura 5.7: Parâmetro ajustado médio para o modelo ER. Nós geramos 1000 grafos do modelo Erdős-Rényi de tamanhos $n=20,50,100,500,1000,10000$, com probabilidades de conectar cada dois vértices $(a) p=0.1,(b) p=0.3,(c) p=0.5,(d) p=0.7 e(e) p=0.9$. Em cada gráfico, os eixos $x e$ $y$ correspondem, respectivamente, ao tamanho do grafo (n) e ao parâmetro ajustado. O parâmetros ajustados médios estão indicados pelos pontos. As barras de erro em preto indicam os intervalos de confiança de $95 \%$. As linhas/pontos azuis correspondem aos resultados baseados na norma $\ell_{1}$ entre a DEE e a densidade espectral assintótica teórica do modelo ER (lei semi-circular). Analogamente, as linhas verdes tracejadas / pontos se referem aos resultados baseados na distribuição acumulada dos autovalores. As linhas vermelhas pontilhadas indicam o valor esperado.

\section{Outros cenários}

Nós também consideramos cenários nos quais as condições dos Teoremas 2 e 3 podem não ser satisfeitas e/ou a densidade espectral assintótica não existe ou é desconhecida analiticamente, como nos modelos GE, WS, e BA. Nesse caso, para avaliar o desempenho do procedimento para ajuste de modelo baseado na norma $\ell_{1}$, nós estimamos a densidade espectral do modelo por Monte Carlo, como descrito no Algoritmo 1.

Foram gerados 1000 grafos de tamanhos $n=50,100,500$ com parâmetros $r=$ 0.1, 0.3, 0.5, 0.7, 0.9 para o modelo GE. Para os modelos WS e BA, consideramos $n=$ $50,100,500,1000$, com parâmetros $p_{r}=0.1,0.3,0.5,0.7,0.9$ e $p_{s}=1.1,1.3,1.5,1.7,1.9$, respectivamente. Na Figura 5.9, nós mostramos o parâmetro ajustado médio e o intervalo de confiança de $95 \%$ para o modelo GE. Resultados obtidos para os modelos WS e BA são exibidos nas Figuras 5.10 e 5.11, respectivamente. As linhas/pontos azuis correspondem aos resultados baseados na norma $\ell_{1}$ entre a DEE do grafo observado e a DEE média do modelo, estimada como descrito no Algoritmo 1. Similarmente, as linhas tracejadas / pontos verdes correspondem aos resultados baseados na distribuição acumulada empírica dos autovalores. As linhas pontilhadas em vermelho indicam o valor verdadeiro do parâmetro. 
(a) $d=3$

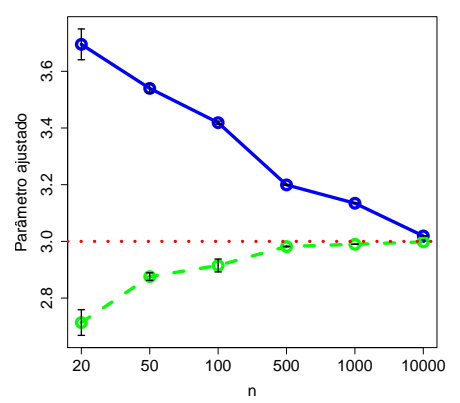

(b) $d=5$

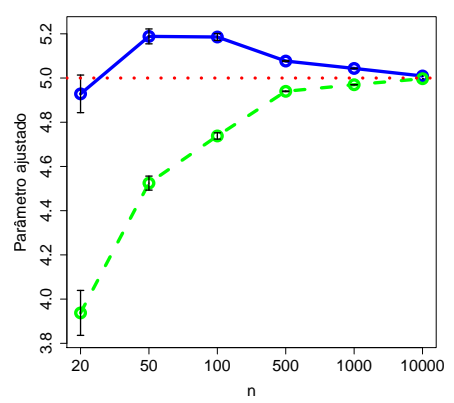

(c) $d=10$

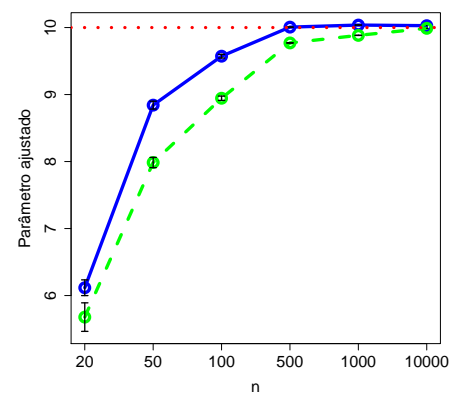

(d) $d=\sqrt{n}$

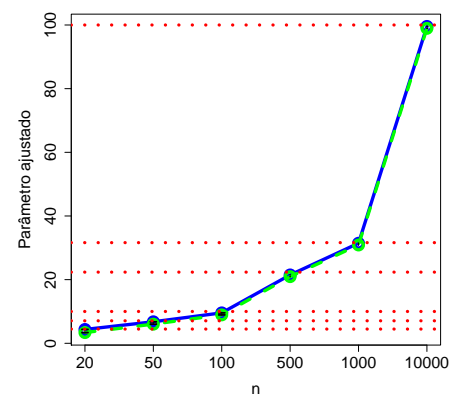

- Média DEE

- Média DAA

.. Valor esperado

Figura 5.8: Parâmetro ajustado médio para o modelo DR. Nós geramos 1000 grafos aleatórios $d$ regulares de tamanhos $n=20,50,100,500,1000,10000$, com graus $(a) d=3,(b) d=5,(c) d=10 e$ (d) $d=\sqrt{n}$. Em cada gráfico, os eixos $x$ e y correspondem, respectivamente, ao tamanho do grafo ( $n$ ) e ao parâmetro ajustado. Os parâmetros ajustados médios estão indicados pelos pontos. As barras de erro em preto indicam os intervalos de confiança de 95\%. As linhas/pontos azuis correspondem aos resultados baseados na norma $\ell_{1}$ entre a DEE e a densidade espectral assintótica teórica do modelo DR (lei de Kesten-McKay). Analogamente, as linhas tracejadas / pontos verdes se referem aos resultados baseados na norma $\ell_{1}$ entre a distribuição acumulada dos autovalores empírica e a distribuição acumulada assintótica teórica. As linhas vermelhas pontilhadas indicam os valores esperados.

Para todos os modelos e abordagens (DEE e DAA) considerados, na maioria dos cenários, nós observamos que o parâmetro ajustado se aproxima do valor verdadeiro à medida que o número de vértices aumenta. Contudo, quando $p_{r}=0.3, r=0.3$ e $p_{s}=1.3$, nós observamos que os parâmetros ajustados ficaram mais distantes do valor esperado para grafos de tamanho $n=100$. Isso sugere que, para alguns parâmetros, os valores estimados se aproximam do verdadeiro apenas quando o grafo é suficientemente grande (por exemplo, $n=500)$. Note que a escala de grandeza do erro depende do modelo. Nos modelos BA e WS, os erros são maiores.

Ao comparar os resultados obtidos com a DEE e DAA, notamos que o estimador baseado na DAA se aproximou mais rapidamente do valor esperado para os modelos GE e BA. Já, para o modelo WS, os resultados das abordagens baseadas em DEE e DAA foram similares.

Além da convergência para o valor esperado, outra propriedade que temos interesse em estudar é a distribuição do estimador de parâmetro, conforme descrevemos na Seção 5.5 . 
(a) $r=0.1$

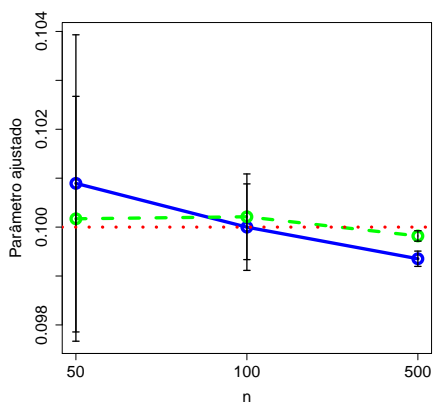

(d) $r=0.7$

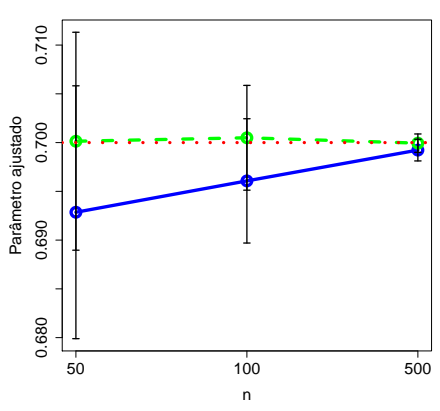

(b) $r=0.3$

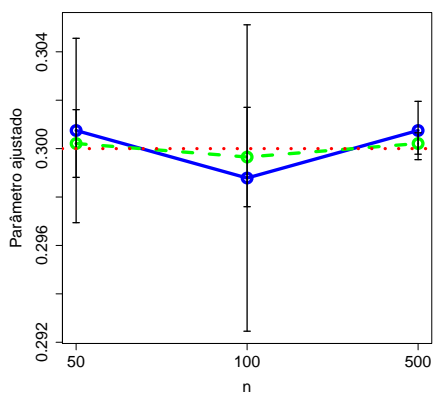

(e) $r=0.9$

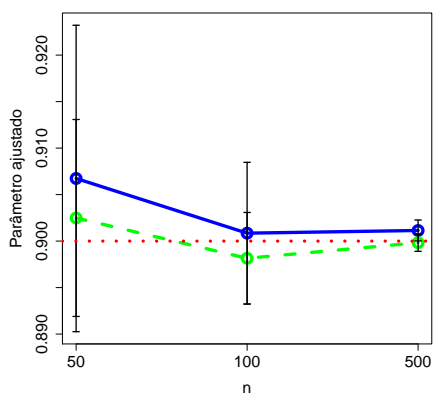

(c) $r=0.5$

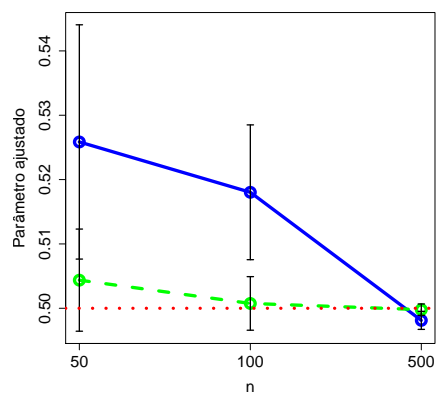

Figura 5.9: Parâmetro ajustado médio para o modelo GE. Nós geramos 1000 grafos aleatórios GE de tamanhos $n=50,100,500$, com raio $(a) r=0.1,(b) r=0.3,(c) r=0.5,(d) r=0.7$, and $(e)$ $r=0.9$. Em cada gráfico, os eixos $x$ e y correspondem, respectivamente, ao tamanho do grafo $(n)$ e ao parâmetro ajustado. Os parâmetros ajustados médios estão indicados pelos pontos. As barras de erro em preto indicam os intervalos de confiança de 95\%. As linhas/pontos azuis correspondem aos resultados baseados na norma $\ell_{1}$ entre a DEE do grafo observado e a DEE média do modelo geométrico. Analogamente, as linhas tracejadas / pontos verdes se referem a resultados baseados na norma $\ell_{1}$ entre a distribuição acumulada empírica dos autovalores do grafo observado e a distribuição média do modelo. As linhas pontilhadas vermelhas indicam o valor esperado. 
(a) $p_{r}=0.1$

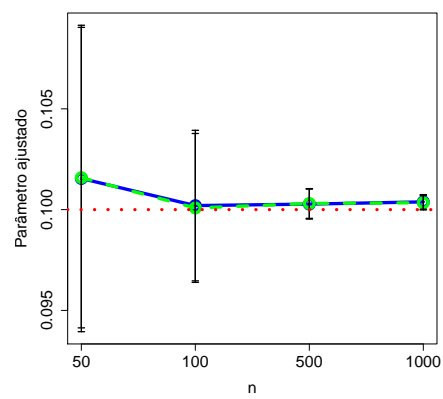

(d) $p_{r}=0.7$

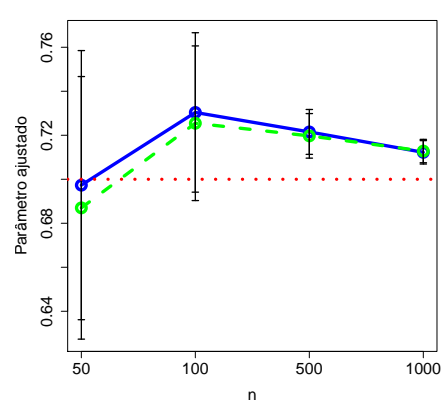

(b) $p_{r}=0.3$

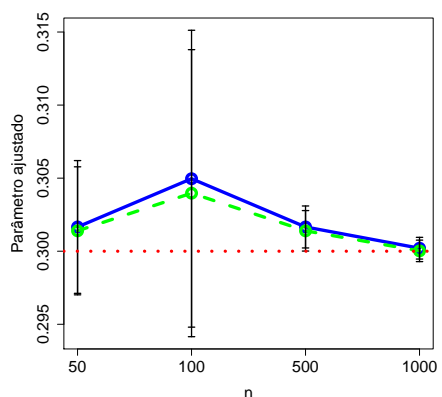

(e) $p_{r}=0.9$

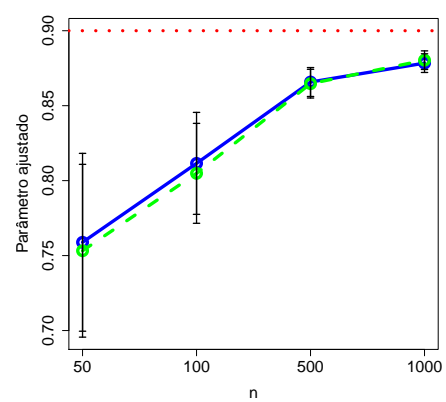

(c) $p_{r}=0.5$

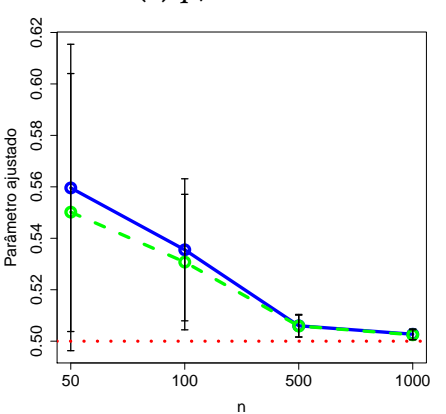

Figura 5.10: Parâmetro ajustado médio para o modelo WS. Nós geramos 1000 grafos aleatórios WS de tamanhos $n=50,100,500,1000$ e probabilidade de reconectar arestas $(a) p_{r}=0.1,(b) p_{r}=0.3$, (c) $p_{r}=0.5,(d) p_{r}=0.7$ e (e) $p_{r}=0.9$. Em cada gráfico, os eixos $x$ e y correspondem, respectivamente, ao tamanho do grafo (n) e ao parâmetro ajustado. Os parâmetros ajustados médios são indicados pelos pontos. As barras de erro em preto indicam os intervalos de confiança de $95 \%$. As linhas / pontos azuis correspondem aos resultados baseados na normal $\ell_{1}$ entre o DEE do grafo observado e o DEE médio do modelo WS. Analogamente, as linhas tracejadas / pontos verdes se referem aos resultados baseados na norma $\ell_{1}$ entre a distribuição acumulada empirica dos autovalores do grafo observado e a distribuição média do modelo WS. As linhas vermelhas pontilhadas indicam os valores esperados. 
(a) $p_{s}=1.1$

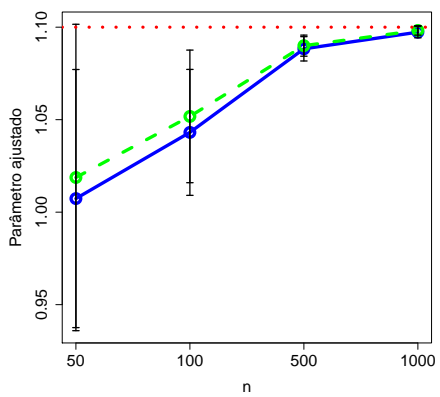

(d) $p_{s}=1.7$

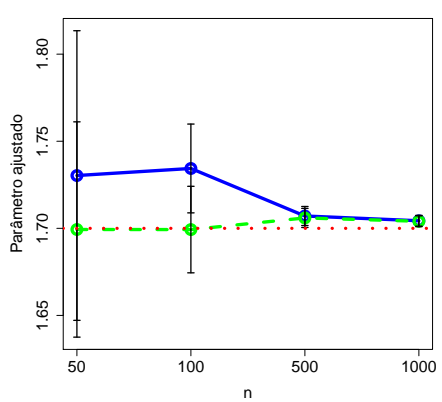

(b) $p_{s}=1.3$

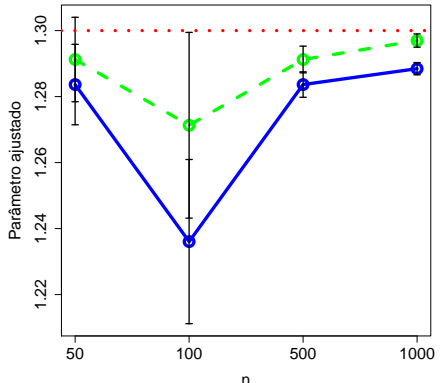

(e) $p_{s}=1.9$

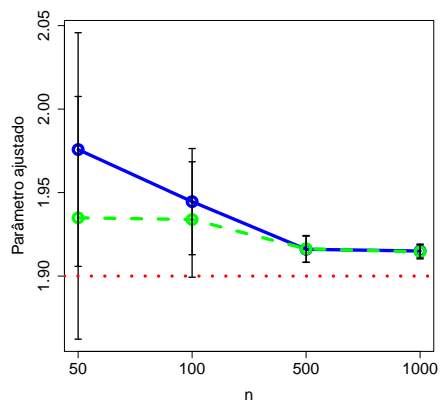

(c) $p_{s}=1.5$

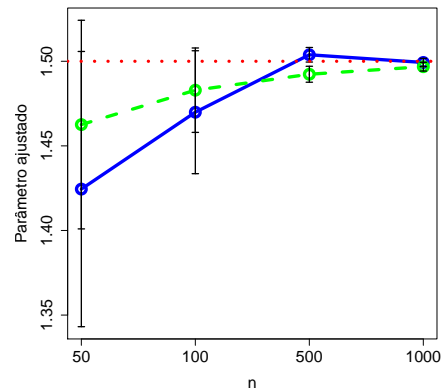

Figura 5.11: Parâmetro ajustado médio para o modelo $B A$. Nós geramos 1000 grafos aleatórios $B A$ de tamanhos $n=50,100,500,1000$ com expoente de escala $(a) p_{s}=1.1,(b) p_{s}=1.3$, (c) $p_{s}=1.5$, $(d) p_{s}=1.7 e(e) p_{s}=1.9$. Em cada gráfico, os eixos $x$ e y correspondem, respectivamente, ao tamanho do grafo (n) e ao parâmetro ajustado. Os parâmetros ajustados médios são indicados pelos pontos. As barras de erro em preto indicam os intervalos de confiança de 95\%. As linhas / pontos azuis correspondem aos resultados baseados na normal $\ell_{1}$ entre o DEE do grafo observado e o DEE médio do modelo BA. Analogamente, as linhas tracejadas / pontos verdes se referem aos resultados baseados na norma $\ell_{1}$ entre a distribuição acumulada empírica dos autovalores do grafo observado e a distribuição média do modelo BA. As linhas vermelhas pontilhadas indicam os valores esperados. IDEM 


\subsection{Distribuição do estimador de parâmetro}

Consideramos dois cenários para o estudo da distribuição dos estimadores de parâmetro baseados na norma $\ell_{1}$ : (i) quando a densidade espectral do modelo é conhecida analiticamente e (ii) quando a densidade do modelo é estimada por Monte Carlo. Ao simular o cenário (i), obtivemos a Figura 5.12, que mostra histogramas dos parâmetros estimados para 1000 grafos de tamanho 10000 gerados pelos modelos $\mathrm{ER}(p=0.5)$ e $\mathrm{DR}(d=5)$. Além disso, geramos gráficos quantis-quantis para comparar a distribuição do estimador com a distribuição normal. Para estimar os parâmetros, as densidades espectrais e as distribuições acumuladas dos autovalores foram comparadas com as funções esperadas pela lei semicircular de Wigner e a lei de Kesten-McKay para grafos ER e DR, respectivamente. Já o cenário (ii) é simulado para grafos GE, BA e WS, para os quais não se conhece uma forma analítica das densidades espectrais assintóticas. Nesse caso, fizemos o ajuste de modelo para 1000 grafos de tamanho 500, gerados com parâmetros $r=0.5$ (GE), $p_{r}=0.5$ (WS) e $p_{s}=1.5$ (BA). Os histogramas dos parâmetros estimados e os gráficos quantis-quantis comparando a distribuição obtida com a distribuição normal são exibidos na Figura 5.13. Em todos os cenários, para estimar a densidade espectral, utilizamos o critério de Silverman para escolha da largura de banda.

Observamos nas Figuras 5.12 e 5.13 que a distribuição do estimador tem um formato semelhante à distribuição normal. Quando utilizamos as distribuições empíricas médias dos autovalores dos modelos GE, WS e BA, vemos na Figura 5.13, que a distribuição está centrada ao redor dos valores esperados. Já, quando utilizamos as distribuições assintóticas teóricas dos autovalores, vemos que, no caso do grafo ER, o valor correto é quase sempre obtido quando o grafo é suficientemente grande $(n=10000)$, com erro inferior a $10^{-7}$. Para o grafo DR, vemos que a variância do estimador é pequena e todos os valores estimados são próximos do valor esperado com erro de até 0.005 para o estimador baseado em DAA e de até 0.025 para o estimador baseado em DEE. 


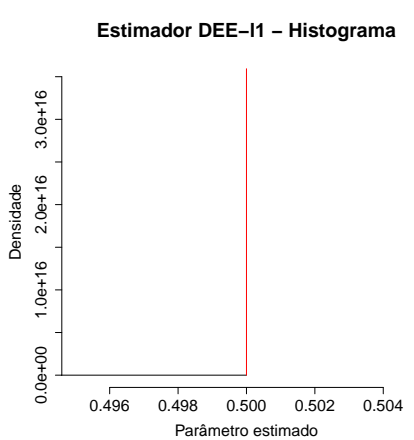

Estimador DEE-I1 - Histograma

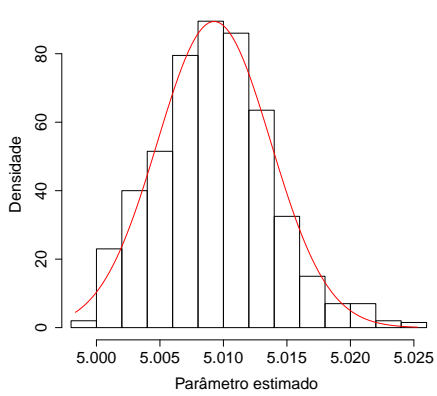

(a) $E R$
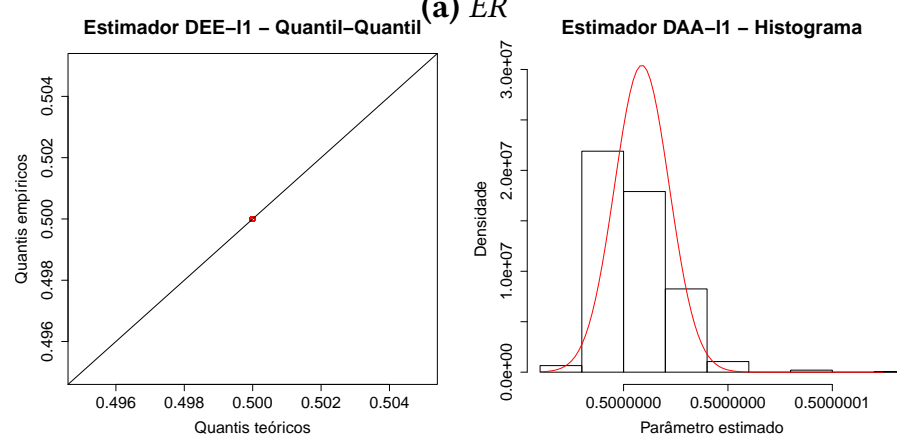

(b) $D R$
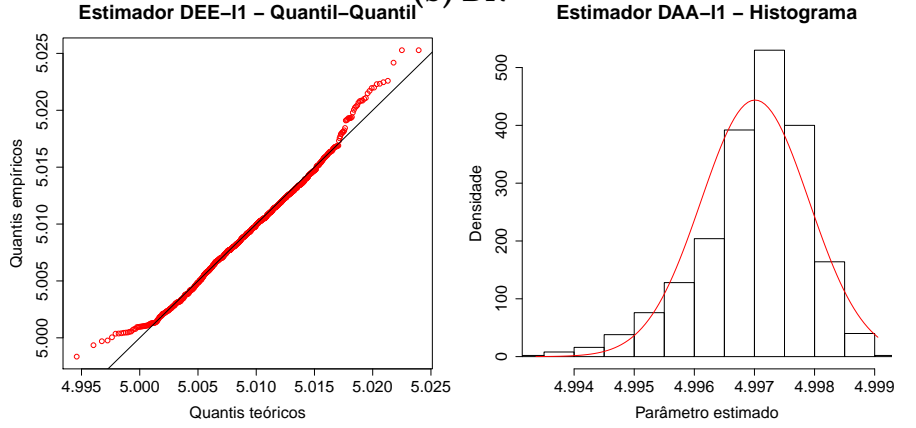

Estimador DAA-I1 - Quantil-Quantil

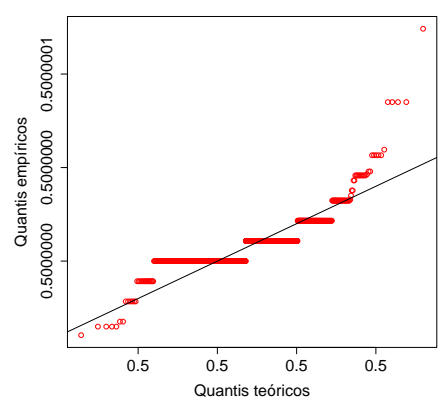

Estimador DAA-I1 - Quantil-Quantil

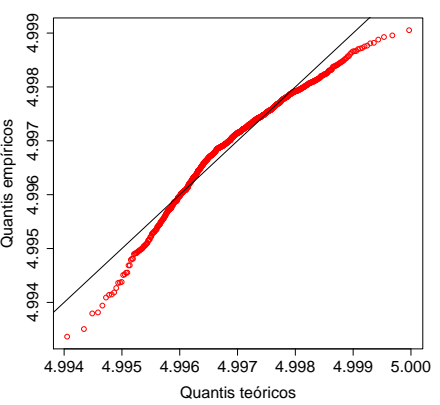

Figura 5.12: Distribuição do estimador de parâmetro para os modelos ER e DR. Foram gerados 1000 grafos de tamanho 10000. Os procedimentos utilizados para estimar o parâmetro se baseiam na norma $\ell_{1}$ entre a distribuição empírica dos autovalores do grafo observado e a distribuição teórica do modelo, segundo a lei semi-circular para o modelo ER e a lei de Kesten-McKay para o grafo DR. Em cada painel mostramos quatro gráficos: os dois primeiros se referem aos resultados obtidos com a densidade espectral (DEE-l1) e os dois últimos aos obtidos com a distribuição acumulada dos autovalores (DAAl1). Os histogramas mostram a distribuição dos parâmetros estimados e a linha vermelha corresponde à distribuição normal. A distribuição dos valores estimados (eixo y) também é contrastada com a normal (eixo $x$ ) no gráfico quantil-quantil. No painel (a), mostramos os resultados para grafos ER gerados com parâmetro $p=0.5$. No painel (b), temos os resultados obtidos para grafos DR com grau $d=5$. 

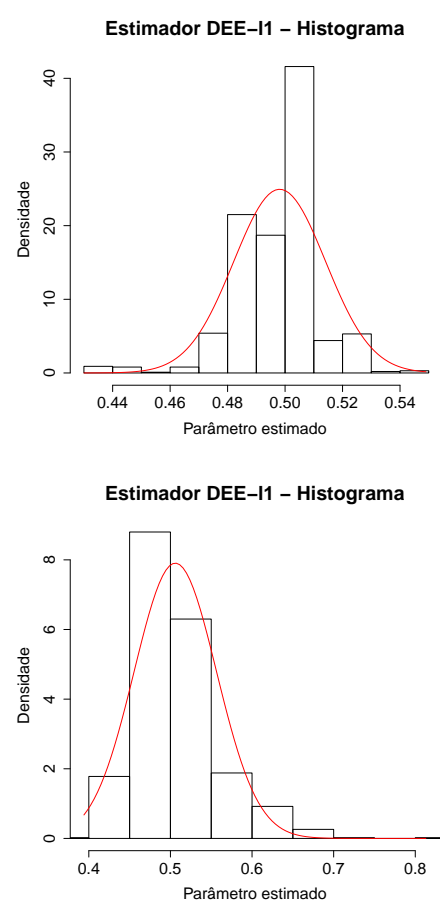

Estimador DEE-11 - Histograma

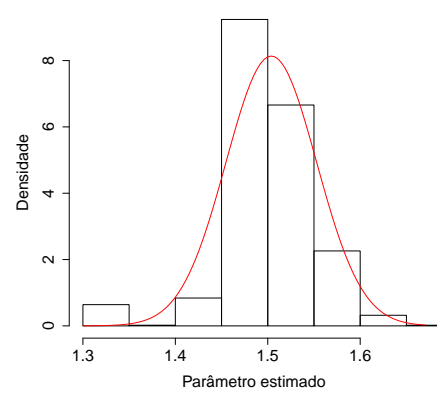

(a) $G E$
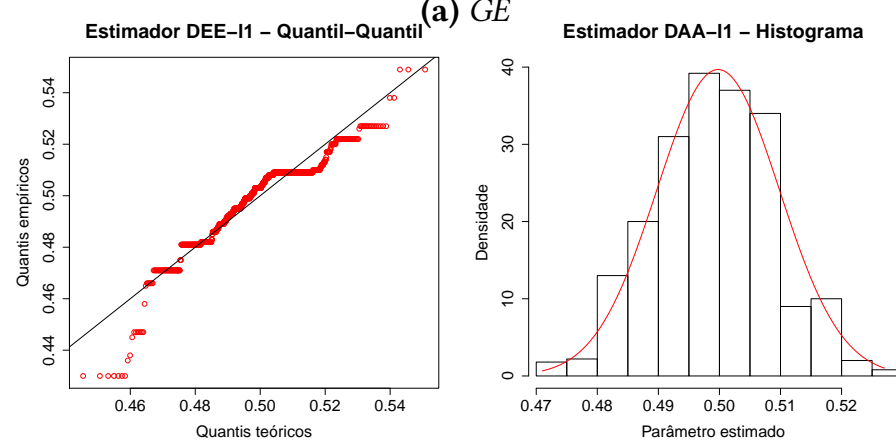

(b) WS
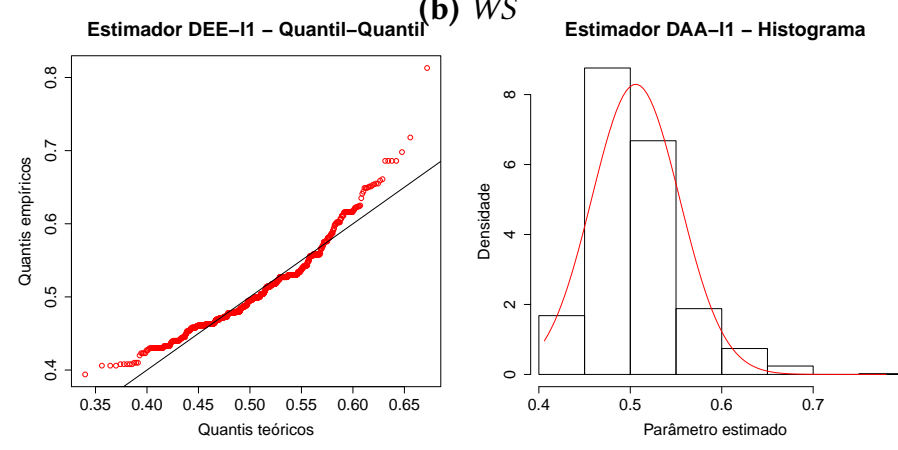

(c) $B A$
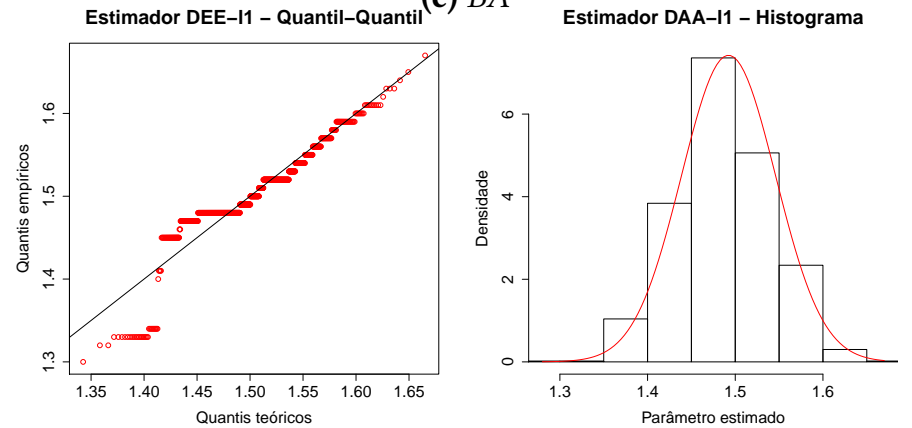
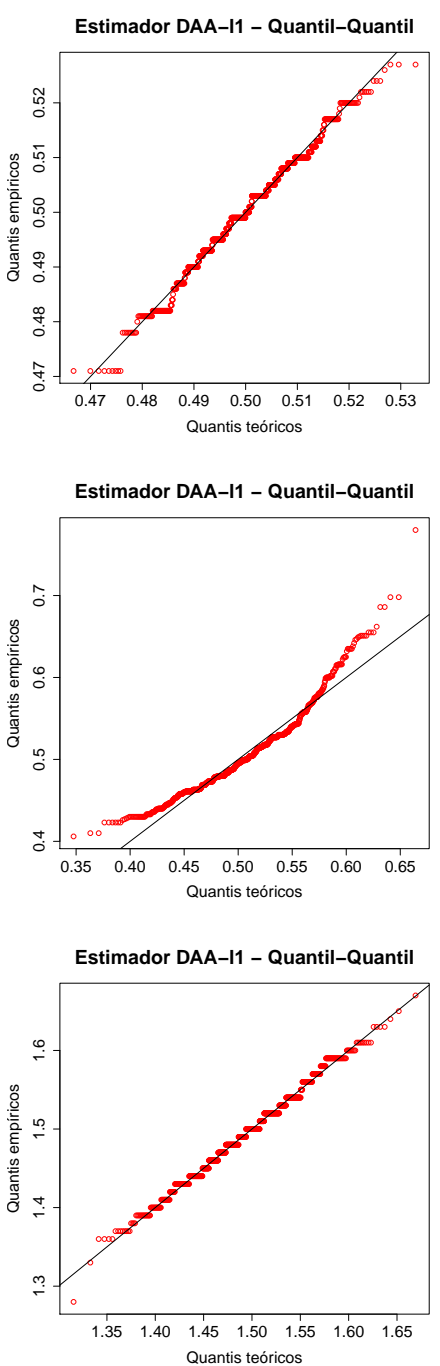

Figura 5.13: Distribuição do estimador de parâmetro para os modelos GE, WS e BA. Foram gerados 1000 grafos de tamanho 50. Os procedimentos utilizados para estimar o parâmetro se baseiam na norma $\ell_{1}$ entre a distribuição empírica dos autovalores do grafo observado e a distribuição empírica média do modelo. Em cada painel mostramos quatro gráficos: os dois primeiros se referem aos resultados obtidos com a densidade espectral (DEE-l1) e os dois últimos aos obtidos com a distribuição acumulada dos autovalores (DAA-l1). Os histogramas mostram a distribuição dos parâmetros estimados e a linha vermelha corresponde à distribuição normal. A distribuição dos valores estimados (eixo y) também é contrastada com a normal (eixo $x$ ) no gráfico quantil-quantil. No paineis (a), (b) $e(c)$, mostramos os resultados para grafos GE (raio $r=0.5$ ), WS (probabilidade de reconectar arestas $\left.p_{r}=0.5\right)$ e BA (expoente de escala $\left.p_{s}=1.5\right)$, respectivamente. 


\subsection{Discussões}

Ao estudarmos o comportamento do procedimento de estimação de parâmetros proposto por TAKAHASHI et al., 2012, notamos que a escolha da largura de banda para estimação da função de densidade influencia os resultados. Além disso, observamos que o desempenho do método depende da escolha entre a função de distribuição utilizada (DEE ou DAA). Já a escolha entre a divergência de Kullback-Leibler e norma $\ell_{1}$ parece não modificar significativamente o desempenho do estimador de parâmetros.

No caso dos grafos ER, GE e WS, o desempenho dos métodos utilizando a norma $\ell_{1}$ entre DEEs e DAAs é similar. Contudo, em relação ao método baseado em DAA, o estimador a partir da DEE se aproxima mais rapidamente do valor esperado em grafos ER e mais lentamente para grafos GE. Já, para o modelo BA, nós observamos um desempenho melhor utilizando a DAA em relação aos resultados obtidos com DEEs estimadas pelos critérios de Silverman e Sturges e similar ao método baseado em DEE estimada por validação cruzada. Essa diferença de desempenho pode ser explicada por erros durante a suavização para estimar as DEEs. Assim, nesse caso, pode ser recomendado o uso da densidade espectral com largura de banda obtida por validação cruzada não viesada ou da distribuição acumulada dos autovalores, sendo que a última tem custo computacional $(O(n))$ inferior ao custo da primeira $\left(O\left(n^{2}\right)\right)$.

Vimos no Capítulo 3 que há cenários em que a DEE de uma sequência de grafos aleatórios converge fracamente (quase certamente) para uma distribuição assintótica. Essas propriedades e o uso da norma $\ell_{1}$ entre DEEs ou DAAs, nos levam aos Teoremas 2 e 3. Se todas as condições são satisfeitas, então o minimizador da norma $\ell_{1}$ entre a distribuição dos autovalores do grafo observado e do modelo converge em probabilidade para o parâmetro verdadeiro à medida que $n \rightarrow \infty$.

De fato, quando as suposições dos Teoremas 2 e 3 são satisfeitas, nossas simulações na Seção 5.4.3 mostram que os parâmetros ajustados se aproximam do valor esperado à medida que o tamanho do grafo aumenta. Quando não temos uma forma analítica para a função de densidade assintótica, nós estimamos a função de densidade a partir de amostragens aleatórias de grafos gerados pelo modelo (como descrito no Algoritmo 1). Nesse caso, o parâmetro estimado também se aproxima do parâmetro verdadeiro à medida que o número de vértices cresce, como mostram os experimentos de simulação descritos em "Outros cenários", na Seção 5.4.3.

Uma das limitações do procedimento descrito no Algoritmo 1 é seu elevado custo computacional, na ordem de $O\left(n^{3}|\tilde{\Theta}|\right)$, onde $|\tilde{\Theta}|$ denota o tamanho da grade para busca dos parâmetros. Contudo, se ao invés de computarmos os autovalores diretamente, estimarmos a contagem de autovalores em cada intervalo (por exemplo, a partir de métodos que aproximam o traço do projetor espectral pelos polinômios de Chebyshev ou funções racionais da matriz de adjacência), a estimação de parâmetros pode ter um custo da ordem de $O\left(n^{2}|\tilde{\Theta}|\right)$ ou $O(n|\tilde{\Theta}|)$ (no caso de grafos esparsos) (Newman et al., 2019; NAPOLI et al., 2016).

Além disso, o procedimento no Algoritmo 1 pode não ser a abordagem mais recomendada para modelos clássicos como o ER e BE, para os quais já existem estimadores com acurácia e eficiência superiores (SNijders e Nowicki, 1997; Ambroise e MATiAs, 
2012). Contudo, o Algoritmo 1 ganha em generalidade. Toda vez que fixamos um modelo paramétrico, podemos construir um procedimento para estimar seu parâmetro e uma medida de divergência entre o modelo e o grafo observado. Isso é particularmente útil no problema de seleção de modelos, conforme discutimos no Capítulo 6. 


\section{Capítulo 6}

\section{Seleção de modelos de grafo aleatório}

O método de estimação de parâmetros descrito no Capítulo anterior, no Algoritmo 1, requer que a distribuição $P$ do grafo aleatório $\mathcal{G}$ pertença a uma dada família de distribuições $\left\{P_{\theta} ; \theta \in \Theta\right\}$. Contudo, na prática não conhecemos essa família (modelo). Assim, dada uma lista de modelos de grafo aleatório, nós escolhemos aquele com melhor ajuste de acordo com algum critério objetivo. Se nós considerarmos modelos de grafo aleatório de mesma complexidade (número de parâmetros), escolhemos o modelo com menor distância entre as densidades espectrais, como proposto por TAKAHASHI et al., 2012. Caso contrário, penalizamos a distância $D$ pelo número de parâmetros.

Formalmente, dada uma lista de modelos de grafo aleatório $\left\{P_{\theta}^{i} ; \theta \in \Theta_{i}\right\}, i \leq N$, TAKAHASHI et al., 2012 propuseram selecionar o modelo com menor AIC (Akaike Information Criterion), como descrito a seguir:

$$
j=\arg \min _{i} 2 K L\left(\mu^{\mathcal{G}}\left(\phi_{x, \sigma}\right), \mu_{\theta}^{i}\left(\phi_{x, \sigma}\right)\right)+2 k_{i},
$$

onde $k_{i}$ é a dimensão de $\Theta_{i}$ e $\mu_{\theta}^{i}$ é a medida de contagem de autovalores assintótica de $P_{\theta}^{i}$.

Se, ao invés da divergência de Kullback-Leibler, considerarmos uma medida de dissimilaridade $D$ e nos restringirmos a modelos de mesma complexidade, podemos adaptar o processo de TAKAHASHi et al., 2012, como descrito no Algoritmo 2.

Para avaliar o desempenho da seleção de modelos baseada na norma $\ell_{1}$ entre densidades espectrais e entre distribuições acumuladas dos autovalores, nós fizemos simulações com grafos de tamanho $n=50,100,500$ gerados pelos modelos Erdős-Rényi (ER) (com parâmetro $p=\beta$ ), geométrico (GE) (com parâmetro $r=\beta$ ), $d$-regular (com parâmetro $d=10 \beta$ ), WattsStrogatz (WS) (com parâmetro $p_{r}=\beta$ ), e Barabási-Albert (BA) (com parâmetro $p_{s}=1+\beta$ ), onde $\beta=0.1,0.5,0.9$. A seleção de modelos foi aplicada a 1000 grafos em cada cenário. As DEEs foram estimadas pelo kernel gaussiano com largura de banda dada pelo critério de Silverman. As matrizes de confusão obtidas pelos métodos baseados na densidade espectral e na distribuição acumulada dos autovalores são exibidas nas Tabelas 6.1, 6.2, 6.3 para 


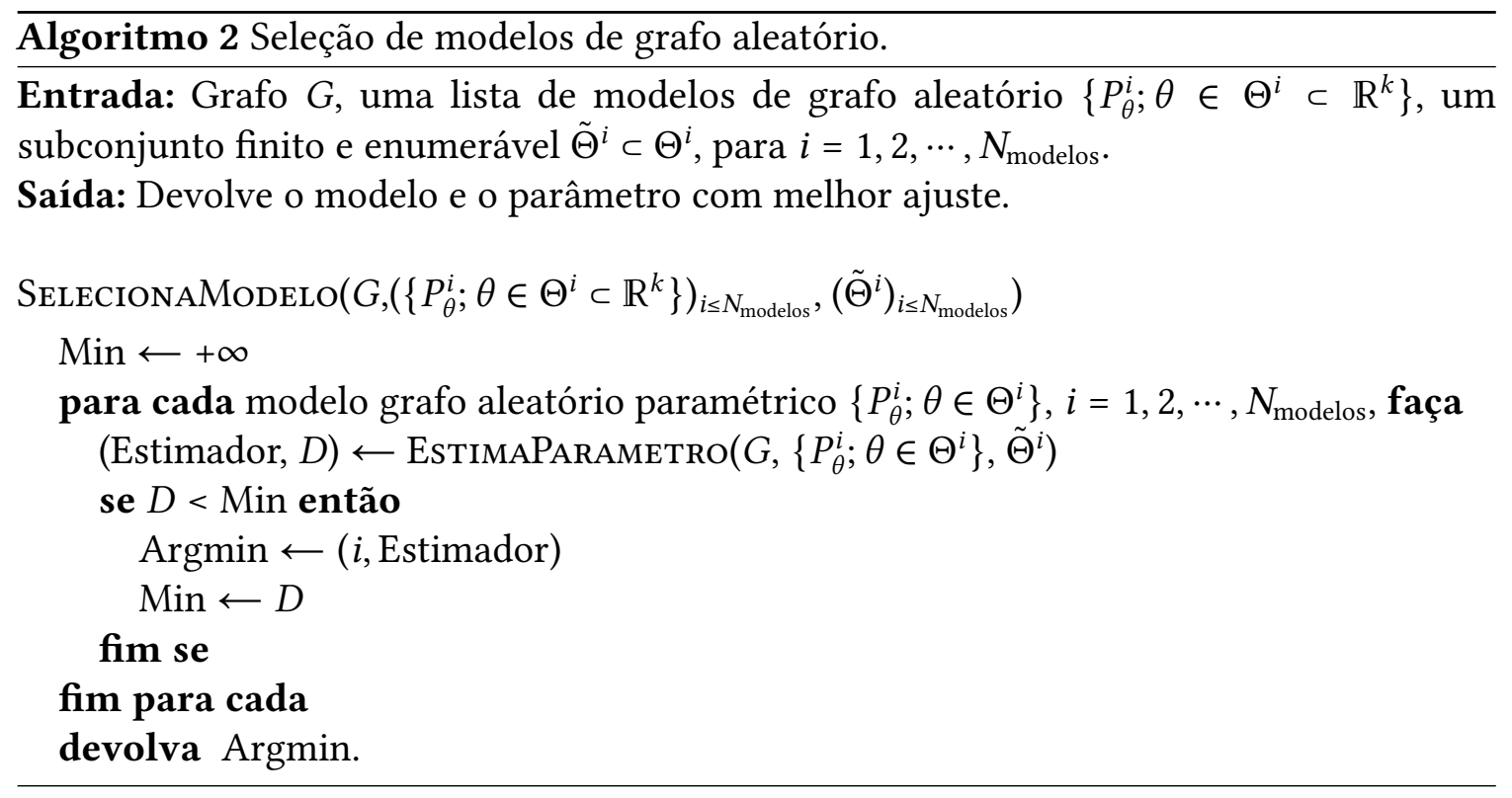

$\beta=0.1,0.5,0.9$, respectivamente.

Tabela 6.1: Seleção de modelos baseada na norma $\ell_{1}$ entre densidades espectrais e entre distribuições acumuladas dos autovalores para grafos gerados com $\beta=0.1$. A tabela mostra matrizes de confusão entre os modelos Erdös-Rényi (ER), geométrico (GE), d-regular (DR), Watts-Strogatz (WS) e Barabási-Albert (BA). Cada célula mostra o número de grafos gerados pelo modelo na linha que foram classificados como o modelo na coluna de acordo com o método baseado na densidade espectral empírica (à esquerda da barra vertical) e na distribuição acumulada empírica dos autovalores (à direita da barra vertical). Para cada modelo, foram gerados 1,000 grafos de tamanhos $n=50,100,500$ como probabilidade de conectar vértices $p=\beta(E R)$, raio $r=\beta(G E)$, grau $d=10 \beta$ $(D R)$, probabilidade de reconectar arestas $p_{r}=\beta(W S)$, e expoente de escala $p_{s}=1+\beta(B A)$.

\begin{tabular}{c|c|ccccc}
\multirow{3}{*}{$n$} & Modelo verdadeiro & \multicolumn{5}{c}{ Modelo selecionado } \\
& ER & DR & GE & WS & BA \\
\hline \multirow{5}{*}{50} & ER & $881 \mid 929$ & $7 \mid 5$ & $0 \mid 0$ & $112 \mid 66$ & $0 \mid 0$ \\
& DR & $0 \mid 0$ & $1000 \mid 1000$ & $0 \mid 0$ & $0 \mid 0$ & $0 \mid 0$ \\
& GE & $4 \mid 20$ & $0 \mid 0$ & $996 \mid 980$ & $0 \mid 0$ & $0 \mid 0$ \\
& WS & $0 \mid 0$ & $0 \mid 0$ & $0 \mid 0$ & $1000 \mid 1000$ & $0 \mid 0$ \\
\hline \multirow{5}{*}{100} & BA & $108 \mid 16$ & $0 \mid 0$ & $3 \mid 0$ & $0 \mid 0$ & $889 \mid 984$ \\
\hline & ER & $993 \mid 998$ & $7 \mid 2$ & $0 \mid 0$ & $0 \mid 0$ & $0 \mid 0$ \\
& DR & $0 \mid 0$ & $1000 \mid 1000$ & $0 \mid 0$ & $0 \mid 0$ & $0 \mid 0$ \\
& GE & $0 \mid 0$ & $0 \mid 0$ & $1000 \mid 1000$ & $0 \mid 0$ & $0 \mid 0$ \\
& WS & $0 \mid 0$ & $0 \mid 0$ & $0 \mid 0$ & $1000 \mid 1000$ & $0 \mid 0$ \\
& BA & $94 \mid 3$ & $0 \mid 0$ & $2 \mid 0$ & $0 \mid 0$ & $904 \mid 997$ \\
\hline \multirow{5}{*}{500} & ER & $994 \mid 998$ & $6 \mid 2$ & $0 \mid 0$ & $0 \mid 0$ & $0 \mid 0$ \\
& DR & $0 \mid 0$ & $1000 \mid 1000$ & $0 \mid 0$ & $0 \mid 0$ & $0 \mid 0$ \\
& GE & $0 \mid 0$ & $0 \mid 0$ & $1000 \mid 1000$ & $0 \mid 0$ & $0 \mid 0$ \\
& WS & $0 \mid 0$ & $0 \mid 0$ & $0 \mid 0$ & $1000 \mid 1000$ & $0 \mid 0$ \\
\hline & BA & $2 \mid 0$ & $0 \mid 0$ & $0 \mid 0$ & $0 \mid 0$ & $998 \mid 1000$ \\
\hline
\end{tabular}

Nós observamos nas Tabelas 6.1, 6.2 e 6.3 que o número de acertos aumenta à medida 
Tabela 6.2: Seleção de modelos baseada na norma $\ell_{1}$ entre densidades espectrais e entre distribuições acumuladas dos autovalores para grafos gerados $\operatorname{com} \beta=0.5$. A tabela mostra matrizes de confusão entre os modelos Erdős-Rényi (ER), geométrico (GE), d-regular (DR), Watts-Strogatz (WS) e Barabási-Albert (BA). Cada célula mostra o número de grafos gerados pelo modelo na linha que foram classificados como o modelo na coluna de acordo com o método baseado na densidade espectral empírica (à esquerda da barra vertical) e na distribuição acumulada empírica dos autovalores (à direita da barra vertical). Para cada modelo, foram gerados 1,000 grafos de tamanhos $n=50,100,500$ como probabilidade de conectar vértices $p=\beta(E R)$, raio $r=\beta(G E)$, grau $d=10 \beta$ $(D R)$, probabilidade de reconectar arestas $p_{r}=\beta(W S)$, e expoente de escala $p_{s}=1+\beta(B A)$.

\begin{tabular}{c|c|ccccc}
\multirow{3}{*}{$n$} & Modelo verdadeiro & \multicolumn{5}{c}{ Modelo selecionado } \\
& ER & DR & GE & WS & BA \\
\hline \multirow{5}{*}{50} & ER & $948 \mid 911$ & $52 \mid 89$ & $0 \mid 0$ & $0 \mid 0$ & $0 \mid 0$ \\
& DR & $1 \mid 0$ & $999 \mid 1000$ & $0 \mid 0$ & $0 \mid 0$ & $0 \mid 0$ \\
& GE & $2 \mid 0$ & $0 \mid 0$ & $998 \mid 1000$ & $0 \mid 0$ & $0 \mid 0$ \\
& WS & $21 \mid 0$ & $0 \mid 0$ & $0 \mid 0$ & $979 \mid 1000$ & $0 \mid 0$ \\
& BA & $64 \mid 3$ & $0 \mid 0$ & $103 \mid 3$ & $0 \mid 0$ & $833 \mid 994$ \\
\hline \multirow{5}{*}{100} & ER & $949 \mid 951$ & $51 \mid 49$ & $0 \mid 0$ & $0 \mid 0$ & $0 \mid 0$ \\
& DR & $0 \mid 0$ & $1000 \mid 1000$ & $0 \mid 0$ & $0 \mid 0$ & $0 \mid 0$ \\
& GE & $0 \mid 0$ & $0 \mid 0$ & $1000 \mid 1000$ & $0 \mid 0$ & $0 \mid 0$ \\
& WS & $6 \mid 0$ & $0 \mid 0$ & $0 \mid 0$ & $994 \mid 1000$ & $0 \mid 0$ \\
& BA & $121 \mid 1$ & $0 \mid 0$ & $125 \mid 1$ & $0 \mid 0$ & $754 \mid 998$ \\
\hline \multirow{5}{*}{500} & ER & $908 \mid 922$ & $92 \mid 78$ & $0 \mid 0$ & $0 \mid 0$ & $0 \mid 0$ \\
& DR & $0 \mid 0$ & $1000 \mid 1000$ & $0 \mid 0$ & $0 \mid 0$ & $0 \mid 0$ \\
& GE & $0 \mid 0$ & $0 \mid 0$ & $1000 \mid 1000$ & $0 \mid 0$ & $0 \mid 0$ \\
& WS & $0 \mid 0$ & $0 \mid 0$ & $0 \mid 0$ & $1000 \mid 1000$ & $0 \mid 0$ \\
\hline & BA & $31 \mid 0$ & $0 \mid 0$ & $11 \mid 0$ & $0 \mid 0$ & $958 \mid 1000$ \\
\hline
\end{tabular}

que o grafo fica maior. O único cenário em que isso não acontece é quando $p=0.5$ para os grafos ER. Nesse caso, alguns grafos ER se confundem com grafos DR. Esse resultado pode ser explicado pelo fato de existirem cenários em que a DEE de grafos ER e DR convergem para a lei semi-circular. Além disso, grafos DR podem ser pensados como um tipo particular de ER nos quais todos os vértices têm mesmo grau $p(n-1)$.

Notamos também que, em todos os cenários, a seleção de modelos de grafos BA teve desempenho superior ao utilizarmos a DAA. Para grafos WS, o desempenho do método baseado em DAA foi superior ou similar ao baseado em DEE. O mesmo acontece com grafos GE, exceto quando $r=0.1$ (Tabela 6.1) e $n=50$.

Concluímos que a norma $\ell_{1}$ entre DEEs e DAAs pode ser utilizada para selecionar modelos de mesma complexidade, tendo um desempenho semelhante ao método proposto por Takahashi e colegas (TAKAHASHi et al., 2012; SANTOs et al., 2016).

No Capítulo a seguir, os grafos aleatórios representam redes funcionais do cérebro. Estamos interessados em entender como o conjunto de parâmetros desses grafos muda com a maior estrutura de matéria branca no cérebro (corpo caloso) no Transtorno do Espectro Autista, além de selecionar o modelo de grafo aleatório que melhor os descreve. 
Tabela 6.3: Seleção de modelos baseada na norma $\ell_{1}$ entre densidades espectrais e entre distribuições acumuladas dos autovalores para grafos gerados $\operatorname{com} \beta=0.9$. A tabela mostra matrizes de confusão entre os modelos Erdős-Rényi (ER), geométrico (GE), d-regular (DR), Watts-Strogatz (WS) e Barabási-Albert (BA). Cada célula mostra o número de grafos gerados pelo modelo na linha que foram classificados como o modelo na coluna de acordo com o método baseado na densidade espectral empírica (à esquerda da barra vertical) e na distribuição acumulada empírica dos autovalores (à direita da barra vertical). Para cada modelo, foram gerados 1,000 grafos de tamanhos $n=50,100,500$ como probabilidade de conectar vértices $p=\beta(E R)$, raio $r=\beta(G E)$, grau $d=10 \beta$ $(D R)$, probabilidade de reconectar arestas $p_{r}=\beta(W S)$, e expoente de escala $p_{s}=1+\beta(B A)$.

\begin{tabular}{c|c|ccccc}
$n$ & \multirow{5}{*}{ Modelo verdadeiro } & \multicolumn{5}{|c}{ Modelo selecionado } \\
& ER & DR & GE & WS & BA \\
\hline \multirow{5}{*}{50} & ER & $1000 \mid 1000$ & $0 \mid 0$ & $0 \mid 0$ & $0 \mid 0$ & $0 \mid 0$ \\
& DR & $56 \mid 4$ & $944 \mid 996$ & $0 \mid 0$ & $0 \mid 0$ & $0 \mid 0$ \\
& GE & $71 \mid 0$ & $0 \mid 0$ & $929 \mid 1000$ & $0 \mid 0$ & $0 \mid 0$ \\
& WS & $133 \mid 22$ & $0 \mid 0$ & $0 \mid 0$ & $867 \mid 978$ & $0 \mid 0$ \\
& BA & $9 \mid 0$ & $0 \mid 0$ & $22 \mid 0$ & $0 \mid 0$ & $969 \mid 1000$ \\
\hline \multirow{5}{*}{100} & ER & $1000 \mid 1000$ & $0 \mid 0$ & $0 \mid 0$ & $0 \mid 0$ & $0 \mid 0$ \\
& DR & $1 \mid 0$ & $999 \mid 1000$ & $0 \mid 0$ & $0 \mid 0$ & $0 \mid 0$ \\
& GE & $0 \mid 0$ & $0 \mid 0$ & $1000 \mid 1000$ & $0 \mid 0$ & $0 \mid 0$ \\
& WS & $124 \mid 59$ & $0 \mid 0$ & $0 \mid 0$ & $876 \mid 941$ & $0 \mid 0$ \\
& BA & $0 \mid 0$ & $0 \mid 0$ & $2 \mid 0$ & $0 \mid 0$ & $998 \mid 1000$ \\
\hline \multirow{5}{*}{500} & ER & $1000 \mid 1000$ & $0 \mid 0$ & $0 \mid 0$ & $0 \mid 0$ & $0 \mid 0$ \\
& DR & $0 \mid 0$ & $1000 \mid 1000$ & $0 \mid 0$ & $0 \mid 0$ & $0 \mid 0$ \\
& GE & $0 \mid 0$ & $0 \mid 0$ & $1000 \mid 1000$ & $0 \mid 0$ & $0 \mid 0$ \\
& WS & $0 \mid 0$ & $0 \mid 0$ & $0 \mid 0$ & $1000 \mid 1000$ & $0 \mid 0$ \\
& BA & $0 \mid 0$ & $0 \mid 0$ & $0 \mid 0$ & $0 \mid 0$ & $1000 \mid 1000$ \\
\hline
\end{tabular}




\section{Capítulo 7}

\section{Relação entre a rede funcional do cérebro e o volume do corpo caloso em Asperger e autismo}

Neste Capítulo apresentamos um problema em neurociência em que estamos interessados em medir a associação entre a rede funcional do cérebro e o volume do corpo caloso em indivíduos com síndrome de Asperger e autismo. Na Seção 7.1, apresentamos a motivação do problema e revisamos a literatura sobre Asperger, autismo e o estudo da conectividade funcional nesses transtornos de desenvolvimento. Em seguida, na Seção 7.2, descrevemos os métodos utilizados para o pré-processamento dos dados e para as análises comparativas entre Asperger e autismo baseadas no maior autovalor do grafo. Os resultados das análises são exibidos na Seção 7.3 e discutidos na Seção 7.4. Por fim, as conclusões são apresentadas na Seção 7.5 .

\subsection{Asperger e autismo}

O transtorno do espectro autista (TEA) é uma doença de neuro-desenvolvimento, em que processos fundamentais de socialização, comunicação e aprendizagem são afetados em diferentes graus (KLIN, 2006). Diagnósticos precisos e intervenção precoce podem amenizar os sintomas (WolfF et al., 2012; Jin et al., 2015; Zwaigenbaum et al., 2015; ZhaO et al., 2018). Contudo, compreender essa doença é muito desafiador, uma vez que ela é bastante heterogênea e envolve diversas funções de alto nível do cérebro (ZHAO et al., 2018). Ainda há muita discussão se todas as variantes de TEA têm as mesmas bases neurais (TsAI, 2013). A quarta edição do Manual Estatístico e Diagnóstico de Transtornos Mentais (DSM IV, do inglês Diagnostic and Statistical Manual of Mental Disorders) inclui a categoria Asperger, na qual indivíduos apresentam dificuldades sociais e/ou comportamento restrito/repetitivo, mas sem atraso no desenvolvimento de comunicação verbal (Duffy et al., 2013). No DSM V, Asperger foi classificada como parte do TEA. Contudo, ainda é incerto se existem diferenças neurobiológicas entre Asperger e autismo (Kwon et al., 2004; TOAL et al., 2010; Jou et al., 2010; JiAO et al., 2011; Duffy et al., 2013; LinColn et al., 1998; TsAi, 2013; Gráinne M. McAlonan et al., 2008; G. M. McAlonan et al., 2009). Assim, nós investigamos as bases 
neurais envolvidas nesses transtornos usando uma abordagem de grafos sobre um grande conjunto de dados contendo dados de ressonância magnética estrutural (sMRI) e funcional (fMRI).

Existem poucos estudos na literatura descrevendo diferenças entre autismo e Asperger. Estruturas cerebrais com alterações reportadas na literatura incluem: corpo caloso médio (Lincoln et al., 1998), matéria branca no lobo frontal médio (ToAl et al., 2010), matéria cinzenta no lobo temporal superior direito estendendo até o giro supramarginal e os lobos parietais inferiores (KwoN et al., 2004), densidade de matéria cinzenta no corpo do giro do cíngulo (Kwon et al., 2004) e dobradura cortical do lobo frontal (Jou et al., 2010). Mais tarde, JiAo et al., 2011 reportaram diferenças genéticas entre autismo e Asperger. Eles encontraram um polimorfismo de nucleotídeo único (SNP rs878960, no gene GABRB3) que parece distinguir Asperger de autismo de alta funcionalidade. Contudo, suas conclusões são limitadas devido ao pequeno tamanho amostral (13 indivíduos com autismo de alta funcionalidade e cinco com Asperger).

Outra abordagem usada para comparar autismo e Asperger é a ressonância magnética funcional (fMRI), que mede o nível de "atividade cerebral" pelo sinal dependente do nível de oxigênio no sangue (sinal BOLD, do inglês blood oxygen level-dependent). BESSELING et al., 2018 encontraram um aumento do sinal BOLD em áreas subcorticais e no córtex cíngulo anterior em TEA, em um conjunto de dados contendo 242 controles, 63 indivíduos com autismo e 62 pessoas com Asperger. Contudo, análises posteriores revelaram que os sinais BOLD elevados foram encontrados apenas em indivíduos com autismo, mas não nas pessoas diagnosticas com síndrome de Asperger.

Finalmente, autismo e Aperger têm sido associados com conectividade funcional anormal (Assaf et al., 2010; Kennedy e Courchesne, 2008; Kennedy, Redcay et al., 2006; Monk et al., 2009; Weng et al., 2010; HAGEN et al., 2013; Jung et al., 2014), que é definida como a correlação temporal de medidas de "atividade cerebral" entre regiões do cérebro (Greicius, 2008; Friston et al., 1993). Duffy et al., 2013 utilizaram conectividade funcional obtida por eletroencefalografia (EEG) para classificar indivíduos como tendo autismo ou Asperger. Eles classificaram corretamente $83.85 \%$ dos indivíduos com autismo e $92.3 \%$ dos indivíduos com Asperger, sugerindo que essas condições apresentam diferenças fisiológicas na conectividade funcional baseada em EEG. Contudo, como no trabalho anterior, as conclusões foram limitadas devido ao pequeno número de indivíduos com Asperger (26 indivíduos).

Nos últimos anos, estudos de EEG e fMRI mostraram que há um elevado nível de conectividade funcional entre os hemisférios esquerdo e direito, formando redes "canônicas" quando o indivíduo está em estado de repouso. Essas redes correspondem a funções críticas do cérebro, como movimento, visão, audição, linguagem, memória episódica, função executiva e detecção de saliência (BISwal et al., 1997; Greicius, 2008; Christian F. Beckmann et al., 2005; Cordes et al., 2000; Fox, Snyder et al., 2005; Fransson, 2005; Greicius et al., 2003; HAMpson et al., 2002; SEeley et al., 2007; Custo et al., 2017).

Em particular, a chamada rede modo padrão é frequentemente estudada em TEA, uma vez que diversos estudos reportaram anomalias nessa rede em relação aos controles (Heuvel e Hulshoff Pol, 2010; Bullmore e Sporns, 2009; Harrison et al., 2007; Rombouts et al., 2009; GARrity et al., 2007; Lowe et al., 2008; MohAmMAd et al., 2009; Whitfield- 
GABRIELI et al., 2009; ZHou et al., 2007). Essa rede "canônica" apresenta um elevado nível de ativação em modo reposo quando comparada à ativação durante execução de tarefas cognitivas (Heuvel e Hulshoff Pol, 2010; Gusnard et al., 2001; Raichle, MacLeod et al., 2001; RAIChle e SNYDER, 2007). A rede modo padrão consiste das regiões (funcionalmente ligadas) córtex/pré-cúneo cíngulo posterior, região frontal medial e região parietal inferior (Heuvel e Hulshoff Pol, 2010; Buckner, Andrews-Hanna et al., 2008; Fox e Raichle, 2007; Fox, Snyder et al., 2005; Fransson, 2005; Greicius, 2008; Gusnard et al., 2001; RAICHLE, MAcLeod et al., 2001; RAichle e SNyder, 2007). Os níveis de atividade e a conectividade funcional entre regiões da rede modo padrão estão relacionados a diversos processos cognitivos humanos (Heuvel e Hulshoff Pol, 2010), como a integração do processamento cognitivo e emocional (Greicius, 2008), devaneio (M. F. MASON et al., 2007) e monitoramento do mundo ao redor (GUSNARD et al., 2001). Assim, para investigar as bases neurais de TEA, uma abordagem natural é analisar como a rede modo padrão muda nesse transtorno.

Usualmente, uma rede funcional do cérebro é representada pela média entre todos os pares de regiões do cérebro. Outras abordagens são baseadas em medidas de propriedades estruturais da rede, como coeficiente de agrupamento, assortatividade e as centralidades grau, proximidade e betweenness (Rubinov e SPORns, 2010). Além disso, algumas medidas de redes com peso nas arestas foram propostas, como a distribuição dos pesos e correlação de pesos de arestas (WANG et al., 2010).

Alternativamente, podemos pensar na rede funcional do cérebro como a realização de um processo aleatório (grafo aleatório). Com base nessa ideia, podemos representar a rede do cérebro pelo conjunto de parâmetros do processo aleatório que a gerou. Fujita, TAKAHASH et al., 2017 propuseram descrever o grafo aleatório pelo maior autovalor da matriz de adjacência, pois seu valor esperado é uma função de parâmetros de modelos de grafo aleatório (ER, DR, GE, WS e BA). Além disso, o maior autovalor é no mínimo o grau médio da rede e no máximo o maior grau. Assim, em redes funcionais do cérebro, o maior autovalor é associado à noção de conectividade da rede e eficiência de comunicação entre as regiões.

Mudanças no maior autovalor de redes funcionais do cérebro podem ser uma consequência de alterações na matéria branca. Em particular, o corpo caloso, que é a maior estrutura de matéria branca do cérebro, desempenha um papel importante na conectividade do cérebro, sobretudo entre os hemisférios esquerdo e direito (ANDERSON et al., 2011; ROLAND et al., 2017).

Estudos sobre TEA têm um interesse particular pelo corpo caloso, uma vez que indivíduos com ausência total ou parcial dessa estrutura apresentam dificuldade sociais que também são observadas em TEA (TrAvers et al., 2015). De fato, alguns estudos reportaram anormalidades no corpo caloso e na conectividade funcional inter-hemisférica em indivíduos com TEA quando comparados com controles (TRAVERs et al., 2015; HARDAN et al., 2000; C. N. VidAL et al., 2006; ANDERson et al., 2011).

Como o corpo caloso desempenha um papel importante na conectividade entre os hemisférios esquerdo e direito da rede modo padrão (e, consequentemente, na conectividade da rede toda), nós investigamos como o maior autovalor da rede modo padrão está associado ao volume de sub-regiões do corpo caloso em autismo e Asperger. Mais especificamente, 
nós queremos testar se esta associação é diferente entre ambos.

\subsection{Métodos}

\subsubsection{Conjunto de dados ABIDE}

Nós obtivemos dados de sMRI e fMRI de 908 indivíduos do consórcio Autism Brain Imaging Data Exchange (ABIDE) (Di MARTINo et al., 2014). De acordo com o repositório do ABIDE, a aquisição de métodos e protocolos foram aprovadas pelas comissões avaliadoras institucionais locais correspondentes, isto é, as comissões avaliadoras e suas regulações na California Institute of Technology (Caltech), Carnegie Mellon University (CMU), Kennedy Krieger Institute (KKI), Ludwig Maximilians University Munich (MaxMun), New York University (NYU), Institute of Living at Hartford Hospital (Olin), Oregon Health and Science University, San Diego State University (SDSU), Social Brain Lab da University Medical Center Groningen and Netherlands Institute for Neuroscience (SBL), Stanford University (Standford), Trinity College (Trinity), University of California (UCLA_1 and UCLA_2), University of Leuven (Leuven_1 and Leuven_2), University of Michigan (UM_1 and UM_2), University of Pittsburgh(Pitt), Utah School of Medicine (USM) e Yale School of Medicine (Yale). A aquisição dos dados também está de acordo com as orientações do Health Insurance Portability and Accountability Act (HIPAA) e os protocolos da 1000 Functional Connectomes Project/International Data-sharing Initiative (http://fcon_1000.projects.nitrc.org/).

Os dados de sMRI foram obtidos na página do ABIDE (http://preprocessed-connectomes-project. org/abide/) (C. CradDOck et al., 2013). O pré-processamento dos dados de ressonância magnética estrutural foi feito com o FreeSurfer (http://surfer.nmr.mgh.harvard.edu/) (Fischl, Salat, Busa et al., 2002; Fischl, SAlat, Kouwe et al., 2004), que inclui os passos: segmentação de estruturas subcorticais, extração de superfícies corticais, estimação da espessura cortical, normalização espacial para o modelo de superfície do FreeSurfer (FsAverage) e parcelamento de regiões corticais. Para medir o volume $\left(\mathrm{mm}^{3}\right)$ de 5 sub-regiões do corpo caloso (posterior, posterior médio, central, anterior médio e anterior), nós usamos a ferramenta mri_segstats disponível no FreeSurfer. A segmentação foi realizada no espaço MNI e seu resultado final é exibido na Figura 7.1.

Para pré-processar os dados de fMRI, nós utilizamos o procedimento Athena (http:// www.nitrc.org/plugins/mwiki/index.php/neurobureau:AthenaPipeline), que inclui passos das ferramentas Analysis of Functional NeuroImages (AFNI) (http://afni.nimh.nih.gov) e fMRIB Software Library (FSL) (http://fsl.fMRIb.ox.ac.uk/fsl/fslwiki/). Os principais passos são: exclusão das 4 primeiras imagens; correção de tempo das fatias; desobstrução do conjunto de dados; correção do movimento; exclusão de regiões não-cerebrais; co-registro da imagem média da imagem anatômica do indivíduo; normalização espacial para o espaço MNI (resolução $4 \mathrm{~mm} \times 4 \mathrm{~mm} \times 4 \mathrm{~mm}$ ); extração das séries temporais de sinal BOLD a partir de matéria branca e fluido cerebrospinal; remoção dos efeitos de movimento, matéria branca, fluido cerebrospinal e tendência utilizando regressão linear múltipla; filtro passabanda temporal $(0.009<f<0.08 \mathrm{~Hz})$; e suavização espacial dos dados filtrados utilizando um filtro gaussiano (FWHM $=6 \mathrm{~mm}$ ).

Nós utilizamos dois atlas diferentes para definir as regiões de interesse (ROIs, do inglês 


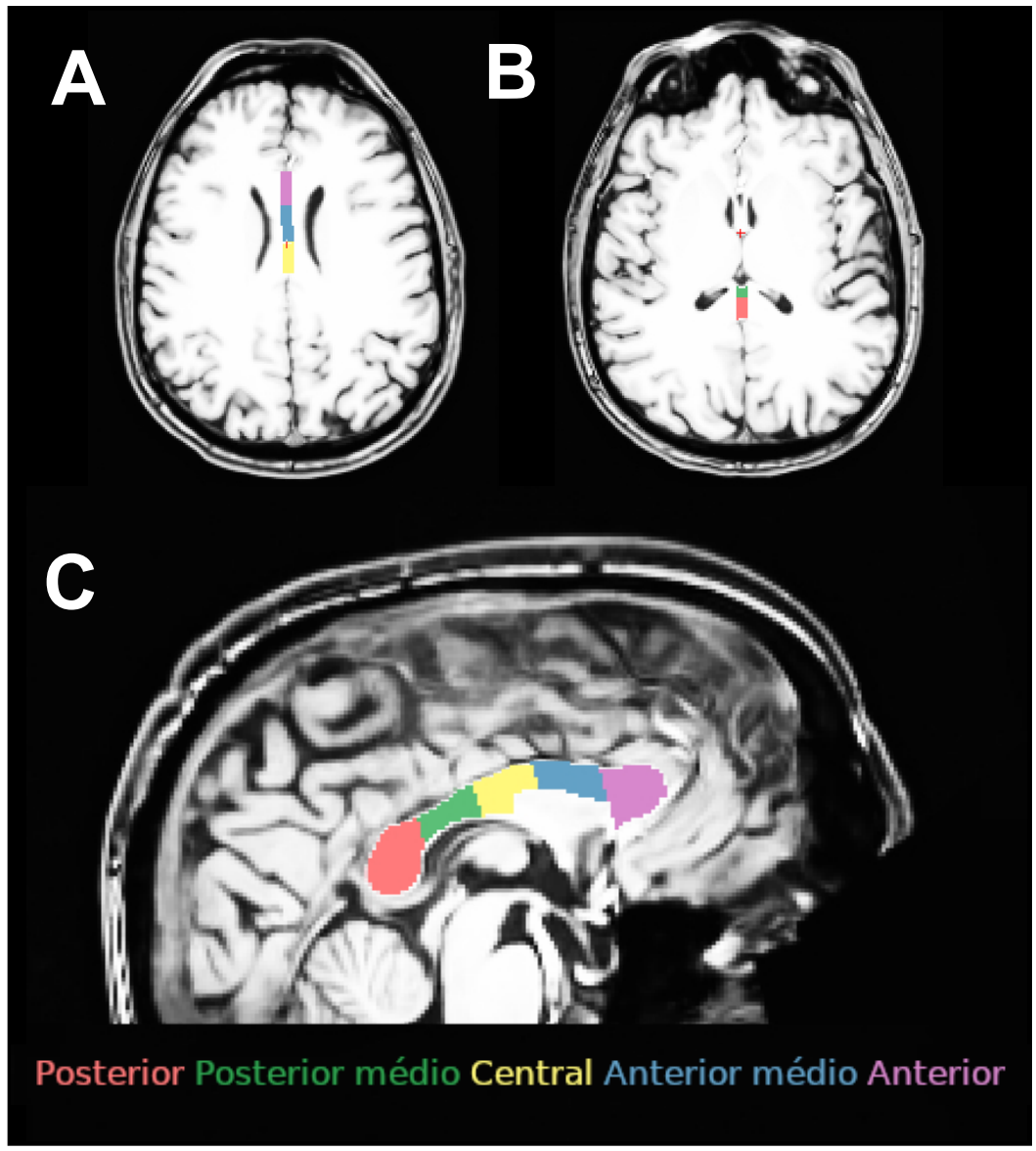

Figura 7.1: Segmentação do corpo caloso em 5 sub-regiões: posterior, posterior médio, central, anterior médio e anterior. O painel (A) mostra as regiões anterior, anterior médio e central em uma fatia axial do cérebro. O painel (B) mostra o corpo caloso posterior médio e posterior em outra fatia axial. Em (C), nós mostramos as cinco sub-regiões numa visão sagital.

regions of interest) a partir dos dados de fMRI: o CC400 (R. C. CRADDOCK et al., 2012) (parcelamento funcional) para dividir o cérebro em módulos funcionais, e o AAL (TzourioMAZOYER et al., 2002) (parcelamento anatômico) para mapear regiões homotômicas (regiões geometricamente correspondentes em cada hemisfério).

Nós obtivemos o atlas CC400 (R. C. CRADDOCK et al., 2012) na página http://preprocessed-connectomes-project.org/abide/Pipelines.html. Este atlas contém um parcelamento funcional com 351 regiões, das quais 35 estavam localizadas nos ventrículos ou estruturas não encefálicas e foram removidas, restando 316 regiões para as análises.

O atlas AAL (Tzourio-Mazoyer et al., 2002), que contém 116 regiões, foi obtido em https://www.nitrc.org/frs/downloadlink.php/3595. Em seguida, selecionamos regiões homotópicas do atlas AAL que pertencem à rede modo padrão. Como os vértices correspondem à regiões do atlas CC400 e não existe uma correspondência exata entre as regiões do CC400 e do AAL, nós filtramos regiões do atlas AAL no qual pelo menos $70 \%$ dos voxels pertencem à rede modo padrão tanto no hemisférico esquerdo quanto no direito (regiões homotópicas): giro frontal medial esquerdo/direito (sobreposição: 76.68\% - esquerdo, $83.53 \%$ 
- direito), córtex orbitofrontal medial esquerdo/direito (sobreposição: 89.8\% - esquerdo, $90.82 \%$ - direito) e pólo temporal médio esquerdo/direito (sobreposição: 95.11\% - esquerdo, $80 \%$ - direito).

Para ambos atlas CC400 e AAL, nós obtivemos a série temporal média dos voxels pertencentes a cada ROI. Finalmente, nós realizamos o procedimento de scrubbing (Power et al., 2012) para remover pontos no tempo em que detectamos uma grande influência da movimentação da cabeça. Como critério para remoção, consideremos os pontos do tempo nos quais a medida de deslocamento do quadro (FD, do inglês framewise displacement) é superior a $0,5 \mathrm{~mm}$ e a medida de mudança na intensidade do sinal entre um volume e o próximo (DVARS) é maior do que 0.5\% $\triangle$ BOLD (Power et al., 2012). Após o scrubbing, nós removemos 94 indivíduos do conjuntos de dados cujas séries temporais ficaram com menos do que 100 pontos (volumes). Dos 814 indivíduos restantes, nós selecionamos 734 que tinham tanto dados estruturais quanto funcionais de ressonância magnética e que passaram no controle de qualidade realizado por três avaliadores (http://preprocessed-connectomes-project.org/abide/quality_assessment.html). O conjunto de dados final foi coletado em 19 laboratórios (Caltech, CMU, Leuven_1, Leuven_2, MaxMun, NYU, Olin, Pitt, SBL, SDSU, Standford, Trinity, UCLA_1, UCLA_2, UM_1, UM_2, USM e Yale) e contém 437 controles, 227 indivíduos diagnosticados com autismo e 70 diagnosticados com Asperger (de acordo com os critérios do DSM IV). Dados sobre a destreza manual estavam disponíveis para 476 indivíduos (89.92\% destros, $8.82 \%$ canhotos, $0.42 \%$ ambidestros e $0.84 \%$ com lateralidade cruzada). Outras informações demográficas são descritas na Tabela 7.1.

Tabela 7.1: Resumo de informações demográficas. Para idade, deslocamento de quadros (FD), tamanho das séries temporais depois do scrubbing (Tamanho série), e proporção de volumes removidos (Prop. volumes removidos), nós mostramos a média \pm o desvio padrão.

\begin{tabular}{ccccccc}
\hline Grupo & Sexo masculino & Sexo feminino & Idade & FD & Tamanho série & Prop. volumes removidos \\
\hline Controle & 347 & 90 & $17.12 \pm 7.29$ & $0.07 \pm 0.12$ & $195.29 \pm 52.98$ & $0.03 \pm 0.09$ \\
Autismo & 202 & 25 & $17.12 \pm 6.96$ & $0.1 \pm 0.13$ & $204.98 \pm 52.59$ & $0.04 \pm 0.09$ \\
Asperger & 59 & 11 & $20.47 \pm 11.06$ & $0.09 \pm 0.11$ & $173.93 \pm 41.66$ & $0.05 \pm 0.1$ \\
\hline
\end{tabular}

\subsubsection{Rede modo padrão}

A rede modo-padrão pode ser pensada como um grafo aleatório $\mathcal{G} \sim P$ não dirigido sobre o conjunto de vértices $V$ que correspondem a $n$ ROIs e o conjunto de arestas $E$ indicando os pares de ROIs conectadas. Se o grafo tem peso nas arestas, para cada aresta $e=\{i, j\} \in E$, associamos um peso não negativo $w(e)$, que corresponde ao coeficiente de correlação de Pearson entre as ROIs $i$ e $j$ (Stephan e Friston, 2009; Smith et al., 2011).

Se o coeficiente de correlação de Pearson entre as ROIs $i$ e $j$ for negativo, então, definimos o peso $w(e)$ da aresta que liga $i$ e $j$ como zero (BUCKNER, SEPUlcre et al., 2009; Rubinov e Sporns, 2010; PAmplona et al., 2015). Nossa escolha foi baseada no fato de que a conectividade funcional negativa pode ser um artefato introduzido durante o préprocessamento (Weissenbacher et al., 2009; Goelman et al., 2014; Chen e Calhoun, 2018). Além disso, a presença de pesos negativos nas arestas geralmente não é recomendada na análise da estrutura de redes (RUBinov e SPORNS, 2010). 
Para selecionar as ROIs (vértices) que fazem parte da rede modo padrão, nós agrupamos as 316 ROIs do atlas CC400 em módulos funcionais, isto é, sub-sistemas especializados em que as ROIs estão densamente conectadas. Primeiramente, nós obtivemos a rede média de todo o cérebro entre os 734 indivíduos (para cada par de ROIs, nós calculamos o coeficiente de correlação de Pearson médio entre todos os indivíduos). Depois, a rede foi dividida em módulos pelo algoritmo de agrupamento espectral (SHI e MALIK, 2000), com o número de grupos selecionado pelo critério da silhueta (RousseEuw, 1987).

\subsubsection{Relação entre a rede modo padrão e o corpo caloso}

Sejam $\alpha$ e $\beta$ duas variáveis aleatórias com observações pareadas $\left(\alpha_{1}, \beta_{1}\right),\left(\alpha_{2}, \beta_{2}\right), \ldots,\left(\alpha_{N}, \beta_{N}\right)$. Sejam $\mathcal{G}_{1}, \mathcal{G}_{2}, \ldots, \mathcal{G}_{N}$ grafos aleatórios correspondendo à rede modo padrão do cérebro de $N$ indivíduos, tais que $\mathcal{G}_{i} \sim P_{\alpha_{i}}$, onde $P_{\alpha_{i}}$ é da família de distribuições $\left\{P_{\theta}, \theta \in \Theta \subset \mathbb{R}^{k}\right\}$ e $i=1,2, \ldots, N$. Assim, podemos descrever cada grafo $\mathcal{G}_{i}$ por meio do parâmetro $\alpha_{i}$.

Podemos formular o problema de testar se a rede modo padrão e o volume do corpo caloso são estatisticamente independentes como um teste de hipótese entre a família de grafos aleatórios $\mathcal{G}$ (rede modo padrão) e a variável aleatória $\beta$ (volume do corpo caloso). O teste pode ser descrito pelas hipóteses:

$H_{0}: \alpha$ e $\beta$ são independentes (hipótese nula).

$H_{1}: \alpha$ e $\beta$ não são independentes (hipótese alternativa).

Para abordar esse problema, quando os parâmetros são desconhecidos, dados os grafos $\mathcal{G}_{1}, \mathcal{G}_{2}, \ldots, \mathcal{G}_{N}$, podemos estimar os parâmetros e testar a independência entre os valores estimados e $\beta_{1}, \beta_{2}, \ldots, \beta_{N}$. Contudo, usualmente não conhecemos o modelo que gerou o grafo e, para alguns modelos, o procedimento de estimação pode ser computacionalmente custoso, como vimos no Capítulo 5.

Um problema similar é abordado por FujITA, TAKAHASHi et al., 2017, em que tanto a variável $\alpha$ quanto a variável $\beta$ correspondem a parâmetros do modelo de grafo aleatório. Eles propuseram utilizar o maior autovalor do grafo ao invés de estimar os parâmetros diretamente. Para diversos modelos de grafo aleatório, o maior autovalor da matriz de adjacência, que denotamos por $\lambda_{1}$, é uma função dos parâmetros. Por exemplo, para o grafo aleatório de Erdős-Rényi, com $n$ vértices e probabilidade $p$ de que dois vértices sejam conectados por uma aresta, o valor esperado de $\lambda_{1}$ é $n p-2 p+1$ (Füredi e KomLós, 1981). Outro exemplo é o grafo $d$-regular aleatório, em que $\lambda_{1}$ é igual ao grau $d$. No caso do grafo geométrico, o maior autovalor converge quase certamente para $r^{k}$, onde $r$ é o raio e $k$ é a dimensão do espaço (BordenAve, 2008). Para o modelo de Watts-Strogatz, há evidências empíricas de que o maior autovalor é uma função dos parâmetros $K$ (número de vértices vizinhos no anel) e $p_{r}$ (probabilidade de reconectar arestas) (VAN Mieghem, 2010). No modelo de Barabási-Albert, o valor esperado de $\lambda_{1}$ é da ordem de $\sqrt{d_{0}} n^{\sqrt{p_{s}-1}}$, onde $d_{0}$ é o menor grau da rede e $p_{s}$ é o expoente de escala (Dorogovtsev et al., 2003).

Fujita, TAKAHASHi et al., 2017 mostraram, por meio de simulações para os grafos GE, WS e BA e teoricamente para ER e DR, que, a partir do maior autovalor dos grafos, é possível capturar a relação entre os parâmetros de grafo aleatório. Os experimentos realizados sugerem que o teste de independência baseado no maior autovalor tem maior poder estatístico do que testes baseados em outras medidas estruturais do grafo como 
transitividade, betweenness, proximidade, autovetor, grau e assortatividade. Assim, neste trabalho, abordamos o problema de interesse por meio do maior autovalor da rede modo padrão do cérebro.

\subsubsection{Seleção de modelos e estimação de parâmetros}

Para interpretar a rede funcional modo padrão, nós selecionamos um modelo de grafo aleatório e ajustamos o modelo para cada grafo observado, conforme procedimento de TAKAHASHi et al., 2012 descrito na Seção 5.1. Nós consideremos os seguintes modelos Erdős-Rényi (Erdős e RÉNyi, 1959), geométrico (Penrose, 2003), Watts-Strogatz (WATTS e Strogatz, 1998) e Barabási-Albert (Barabási e Albert, 1999). Como todos os modelos são grafos sem pesos nas arestas, nós discretizando os grafos considerando diferentes limiares de correlação para remover arestas: $0.1,0.2,0.3,0.4,0.6,0.8$ e 0.9 .

\subsubsection{Comparação entre os grupos}

Nós utilizamos modelos de regressão linear para analisar a associação entre o volume do corpo caloso e o maior autovalor da rede modo padrão. Para cada grupo de indivíduos (autismo, Asperger e controle) e cada sub-região do corpo caloso (posterior, posterior médio, central, anterior médio, anterior), nós ajustamos um modelo linear no qual a variável resposta é o maior autovalor da rede modo padrão e a variável explicativa é o volume de uma região do corpo caloso. Para minimizar os efeitos de idade, sexo, laboratório, deslocamento do quadro e volume intracraniano, nós os incluímos como covariáveis dos modelos lineares. Todas as variáveis quantitativas foram normalizadas para média zero e variância unitária.

Sejam $i$ e $j, i \neq j$, dois grupos de indivíduos (entre autismo, Asperger ou controle). Então, $\beta_{i}$ e $\beta_{j}$ denotam os coeficientes lineares dos grupos $i$ e $j$, respectivamente, para uma dada sub-região do corpo caloso e $S E_{i}$ e $S E_{j}$ denotam os erros padrão correspondentes. Nós comparamos os coeficientes entre os grupos calculando o seguinte $\mathrm{Z}$-valor:

$$
Z=\frac{\beta_{i}-\beta_{j}}{\sqrt{S E_{i}^{2}+S E_{j}^{2}}}
$$

Para corrigir os p-valor por múltiplos testes, nós usamos o método de Bonferroni (Neyman e Pearson, 1928).

\subsection{Resultados}

Após aplicar o algoritmo de agrupamento espectral na rede funcional do cébrebro para dividi-la em diferentes números de módulos, obtivemos o valor máximo da estatística da silhueta quando as regiões do cérebro são separadas em cinco grupos, como mostra a Figura 7.2. Assim, nós agrupamos as 316 regiões do cérebro em cinco sub-sistemas: sensoriomotor, visual, modo padrão, cerebelar, e frontoparietal (Figura 7.3).

A rede modo padrão resultante contém 77 regiões e é similar àquelas obtidas por outros estudos usando diferentes abordagens (Heuvel e Hulshoff PoL, 2010), como ICA 


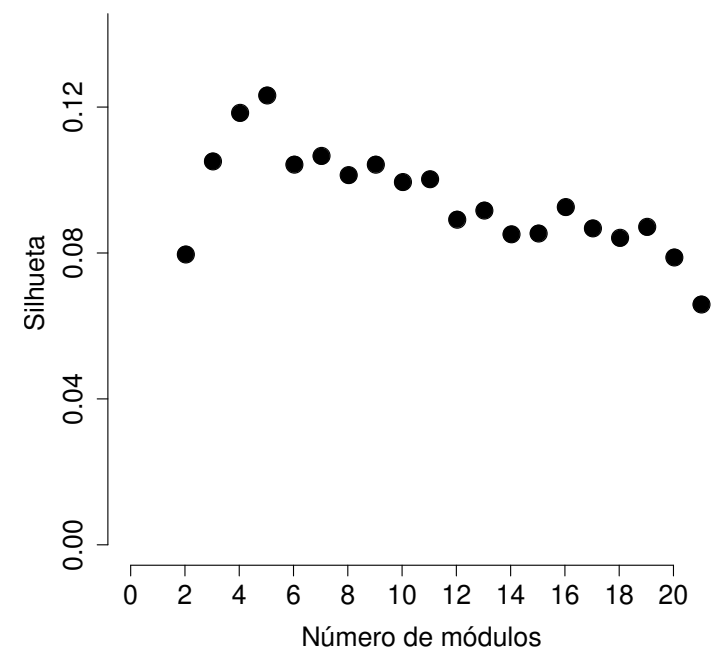

Figura 7.2: Silhueta dos agrupamentos da rede de funcional do cérebro. Os eixos $x$ e y correspondem ao número de módulos e à estatística da silhueta, respectivamente.

(análise de componente independente) (Christian F. BECKMAnn et al., 2005; Damoiseaux, Rombouts et al., 2006; DAmoiseaux, C. F. Beckmann et al., 2008), algoritmos baseados em semente (GREICIUs et al., 2003; WhitfiELD-GABRiELI et al., 2009) e outros algoritmos de agrupamento (Heuvel, Rene Mandl et al., 2008; Heuvel, René Mandl et al., 2008).

Nós analisamos se a associação entre a rede modo padrão e o volume de cada sub-região do corpo caloso (posterior, posterior médio, central, anterior médio e anterior) é igual entre autismo e Asperger. Na Tabela 7.2, nós observamos que a associação é significativamente diferente apenas na região anterior do corpo caloso (p-valor ajustado por Bonferroni: 0.02). Além disso, a associação foi significativa en Asperger (coeficiente $=0.341$, p-valor: 0.013), mas não no autismo (coeficiente $=-0.094$, and p-valor: 0.204). Nós mostramos todos os coeficientes estimados para autismo e Asperger, respectivamente, nas Tabelas A.1 e A.2, no Apêndice A.

Tabela 7.2: Comparação da associação entre a rede modo padrão e o volume de sub-regiões do corpo caloso em autismo e Asperger. Nós testamos se a associação entre a conectividade funcional (maior autovalor) da rede modo padrão e o volume de regiões do corpo caloso (posterior, posterior médio, central, anterior médio e anterior) é igual em ambos grupos. Cada célula contém p-valores nominais e, em parênteses, os valores ajustados para múltiplos testes pelo método de Bonferroni. Nós mostramos os p-valores ajustados significativos em negrito (com um nível de significância de 0.05).

\begin{tabular}{ccccc}
\hline & \multicolumn{3}{c}{ Corpo caloso } \\
Posterior & Posterior médio & Central & Anterior médio & Anterior \\
\hline $0.230(1.00)$ & $0.413(1.00)$ & $0.039(0.19)$ & $0.266(1.00)$ & $\mathbf{0 . 0 0 4 ( 0 . 0 2 )}$ \\
\hline
\end{tabular}

Após realizar uma análise similar nos controles, nós observamos que a associação entre a rede modo padrão e o volume do corpo caloso anterior não é significativamente diferente do autismo ( $p$-valor $=0.313)$, mas é diferente de Asperger $(p$-valor $=0.024)$. Similarmente ao grupo autista, nos controles essa associação não é significativa (coeficiente $=-0.003$, 


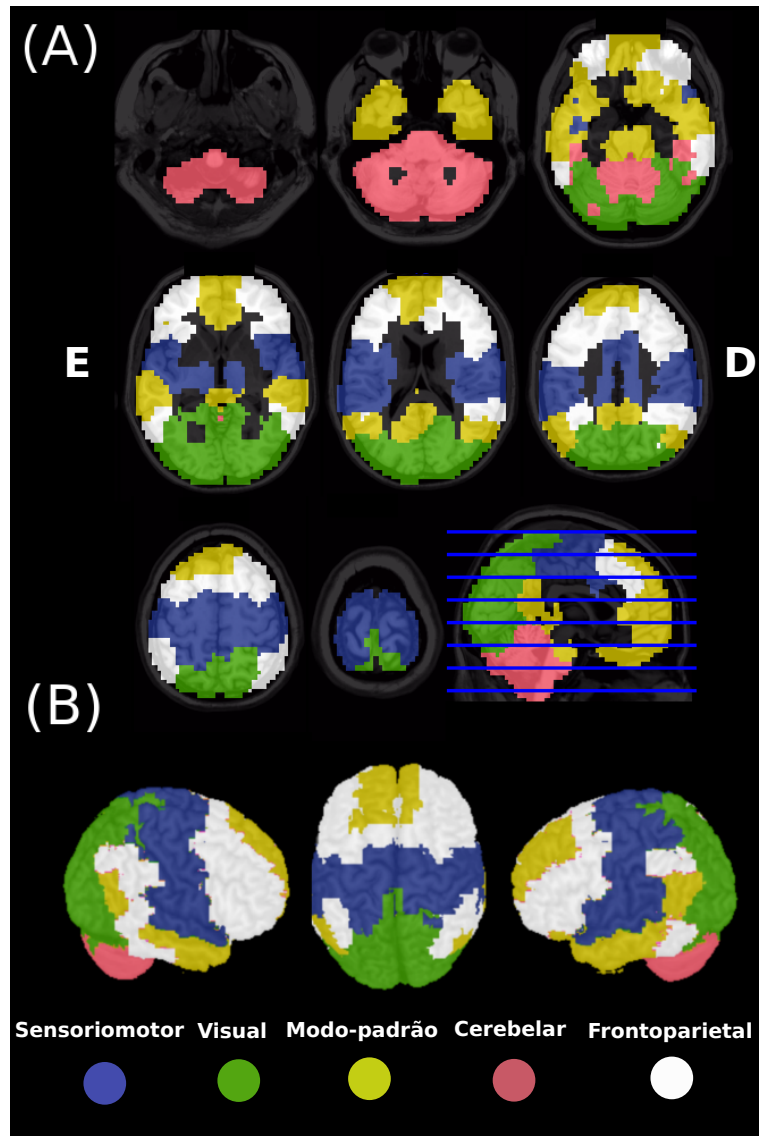

Figura 7.3: Mapeamento dos módulos funcionais do cérebro. Os painéis $(A)$ e (B) representam os cortes axiais e representação em $3 D$ do cérebro inteiro, respectivamente. Cada cor representa um módulo funcional (sensoriomotor, visual, modo padrão, cerebelar e frontoparietal). Os hemisférios esquerdo e direito estão indicados por " $E$ " e " $D$ ", respectivamente.

$\mathrm{p}$-valor $=0.951)$. Os coeficientes e $\mathrm{p}$-valores obtidos para as demais covariaveis estão na Tabela A.3, no Apêndice A.

A relação entre a rede modo padrão (maior autovalor) e o volume do corpo caloso anterior em cada grupo (controle, autismo e Asperger) é ilustrada na Figura 7.4A. Nós também exibimos os valores preditos e o intervalo de confiança de $95 \%$. A reta ajustada para Asperger parece distante da reta ajustada para controles ou para o grupo autista.

Se pensarmos na rede modo padrão como um grafo aleatório gerado a partir de um dos modelos da nossa lista pré-definida (ER, GRG, WS e BA), então o maior autovalor pode ser pensado como uma medida monotonicamente associada ao parâmetro do modelo (FujitA, TAKAHASHI et al., 2017). Assim, para interpretar as mudanças na rede modo padrão, nós selecionamos e ajustamos o modelo que melhor descreve a rede modo padrão, como descrito na Seção 7.2.4.

O modelo que apresentou melhor ajuste foi o modelo de grafo aleatório geométrico, seguido pelo modelo de Watts-Strogatz, o que é consistente com estudos prévios descrevendo características de mundo pequeno e de grafo geométrico compartilhadas com redes funcionais do cérebro (Micheloyannis et al., 2006; BAssett e Bullmore, 2006; Kuchaiev 
et al., 2009). A rede foi discretizada removendo arestas com correlação menor do que 0.5. Foram considerados diferentes limiares $(0.1,0.2,0.3,0.4,0.6,0.8$, e 0.9$)$, mas os resultados não mudaram: nós continuamos a selecionar o modelo geométrico. A única exceção foi para o limiar 0.7. Nesse caso, o modelo de Barabási-Albert foi selecionado, seguido pelo modelo geométrico com uma pequena diferença da divergência de Kullback-Leiber. Assim, consideremos o modelo geométrico o que apresentou melhor ajuste. Espera-se que o raio estimado para a rede modo padrão esteja associado ao maior autovalor, uma vez que o maior autovalor do grafo geométrico converge quase certamente para o raio ao quadrado (BordenAVE, 2008).

Para interpretar os resultados em termos do modelo de grafo aleatório geométrico, nós refizemos as análises anteriores utilizando o raio estimado. Os resultados se mantiveram: a associação entre o volume do corpo caloso anterior e o raio do modelo geométrico é significativamente maior em Asperger do que em autismo ( $\mathrm{p}$-valor $=0.026$ ) e do que no grupo controle $(\mathrm{p}$-valor $=0.017)$. Além disso, como esperado, não há evidência estatística que indique diferença entre os controles e o grupo autista $(p$-valor $=0.981)$. A comparação entre os grupos é ilustrada na Figura 7.4B. A associação entre o raio do modelo geométrico e o volume do corpo caloso anterior não é significativa em controles (coeficiente $=-0.006$, $\mathrm{p}$-value $=0.906)$, nem em autismo (coeficiente $=-0.004$, $\mathrm{p}$-valor $=0.957)$, mas é significativa em Asperger $($ coeficiente $=0.357$, $\mathrm{p}$-valor $=0.016$ )

Para identificar que parte da rede modo padrão tem uma associação aumentada com o volume do corpo caloso em Asperger, nós testamos se a conectividade funcional de regiões homotômicas do atlas AAL que pertencem à rede modo padrão (giro frontal medial esquerdo/direito, córtex orbitofrontal medial esquerdo/direito e pólo temporal médio esquerdo/direito) é igualmente associada ao volume do corpo caloso anterior em autismo e em Asperger. Os p-valores nominais e ajustados por correção de Bonferroni (em parênteses) obtidos para o giro frontal medial, o córtex orbitofrontal medial e o pólo temporal médio são 0.820 (1.00), 0.001 (0.003) e 0.535 (1.00), respectivamente. Portanto, o córtex orbitofrontal medial foi a única região na qual a associação foi significativamente diferente entre autismo e Asperger. A associação foi significativa em Asperger (coeficiente $=0.387, \mathrm{p}$-valor $=0.003$ ), mas não foi significativa em autismo (coeficiente $=-0.091, \mathrm{p}$-valor $=0.214$ ).

Em controles, a relação entre a conectividade inter-hemisférica do córtex orbitofrontal medial $(\mathrm{CIH})$ e o volume do corpo caloso anterior foi significativamente diferente de Asperger ( $\mathrm{p}$-valor da comparação dos coeficientes $=0.001$ ), mas não do autismo ( $\mathrm{p}$-valor $=$ 0.673 ). Essa associação não foi significativa no grupo controle (coeficiente $=-0.053$, $p$-valor $=0.313$ ). A relação entre o volume da região anterior do corpo caloso e a $\mathrm{CIH}$ em cada grupo é ilustrada na Figura 7.4C.

Além de comparar a associação entre o volume da parte anterior do corpo caloso e medidas de conectividade funcional entre os grupos, nós comparamos os valores médios de cada variável: volume do corpo caloso anterior (Figura 7.5A), maior autovalor da rede modo-padrão (Figure 7.5B), raio do modelo de grafo aleatório geométrico (Figura 7.5C) e conectividade funcional entre o córtex orbitofrontal medial esquerdo e direito (Figura 7.5D). Nós ajustamos um modelo de análise de covariância (ANCOVA) com covariáveis idade, sexo, laboratório, deslocamento de quadros (FD) e volume intracraniano. 
O maior autovalor da rede modo padrão (p-valor $=0.025)$ e o raio estimado do modelo geométrico $(\mathrm{p}$-valor $=0.048)$ são significativamente diferentes entre os grupos. Nós observamos na Figura 7.5B-C que a conectividade funcional da rede modo padrão é menor no grupo autista. Contudo variações no volume do corpo caloso anterior, (p-valor $=0.428)$, e na conectividade funcional entre o córtex orbitofrontal esquerdo e direito ( $\mathrm{p}$-valor $=0.230$ ) não foram significativas, como mostramos na Figura 7.5A,D. 
Autismo versus Asperger
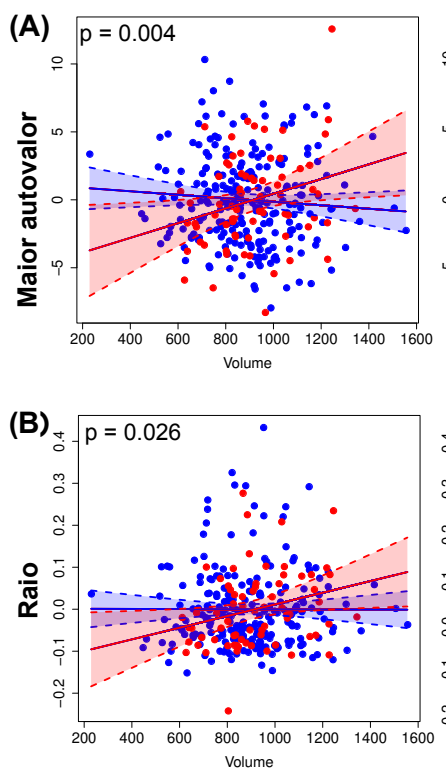

(C)

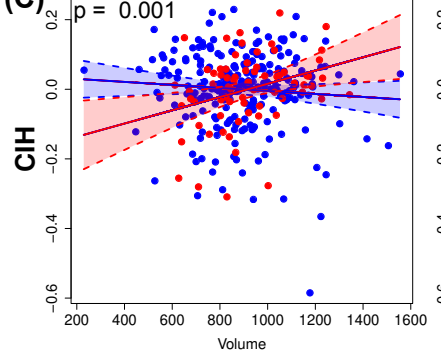

Autismo versus Controles
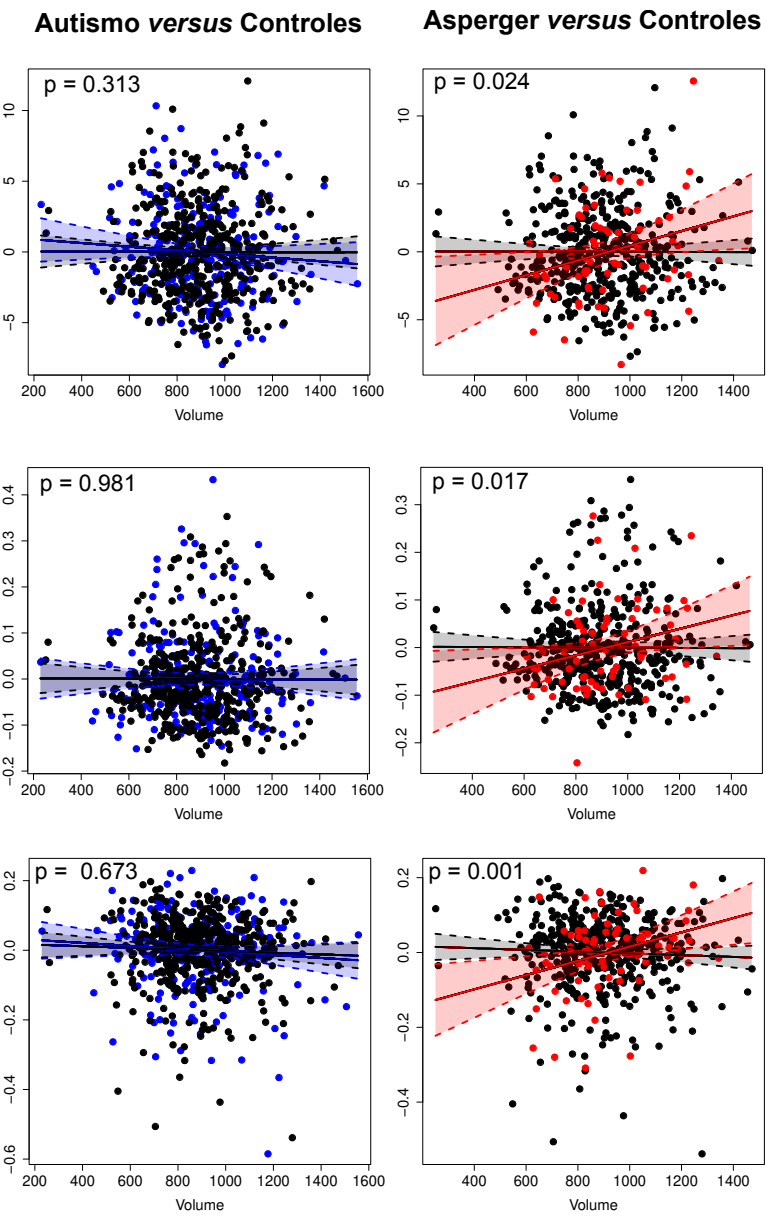

Legenda

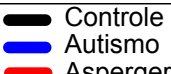

Figura 7.4: Gráficos de dispersão entre o volume da rergião anterior do corpo caloso e medidas de conectividade funcional. Cada medida de conectividade é representada pelos resíduos dos modelos lineares nos quais a conectividade funcional é a variável resposta, o volume do corpo caloso anterior é a variável explicativa e laboratório, sexo, idade, deslocamento de quadros (FD) e volume intracraniano são covariáveis. Em cada gráfico de dispersão, os eixos x e y correspondem, respectivamente, ao volume do corpo caloso anterior e à medida de conectividade funcional: (A) maior autovalor, $(B)$ raio do modelo geométrico e (C) conectividade inter-hemisférica $(C I H)$ do córtex orbitofrontal medial. Os pontos pretos, azuis e vermelhos representam controles, indivíduos com autismo e pessoas diagnosticadas com sindrome de Asperger, respectivamente. Os modelos de regressão linear foram ajustados para cada grupo: controle (linha sólida preta), autismo (linha sólida azul) e Asperger (linha sólida vermelha). Na primeira, segunda e terceira colunas, nós comparamos autismo e Asperger, autismo e controle, e Asperger e controles, respectivamente. Os pvalores (p) para comparar os coeficientes entre cada par de grupos são exibidos em cada gráfico na parte superior esquerda. Nos controles (reta preta), nós obtivemos um coeficiente de -0.003 (p-valor $=0.951),-0.006(p$-valor $=0.906), e-0.053(p$-valor $=0.313)$ para o maior autovalor, raio do modelo geométrico e CIH, respectivamente. No grupo autista, (reta azul), os coeficientes estimados são -0.094 ( $p$-valor $=0.204),-0.004(p$-valor $=0.957) e-0.091(p$-valor $=0.214) . N a$ sindrome de Asperger (reta em vermelho), nós obtivemos os coeficientes 0.341 ( $p$-valor $=0.013), 0.357(p$-valor $=0.016), e$ 0.387 ( $p$-valor $=0.003)$. As linhas tracejadas em preto, azul e vermelho representam os intervalos de confiança de $95 \%$ ao redor dos valores preditos pelo modelo de regressão linear em controles, autismo e Asperger, respectivamente 
(a) Volume médio do corpo caloso anterior.

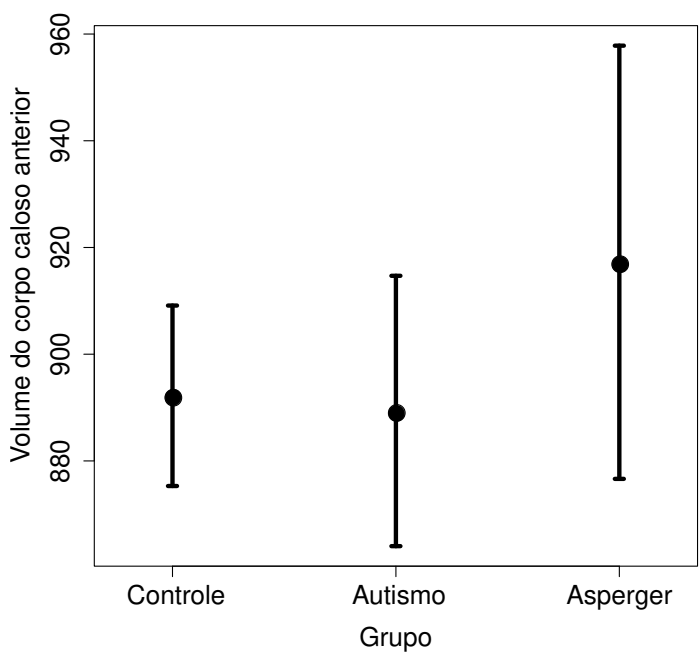

(c) Média do raio estimado do grafo aleatório geométrico.

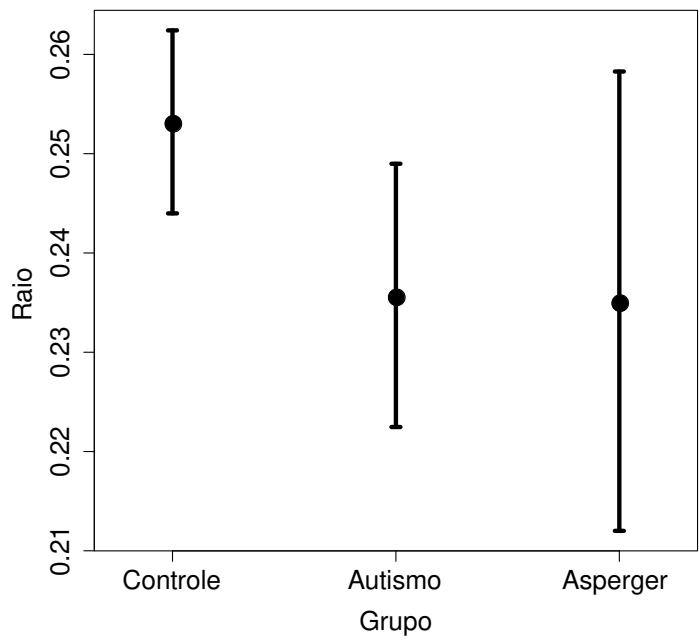

(b) Média do maior autovalor.

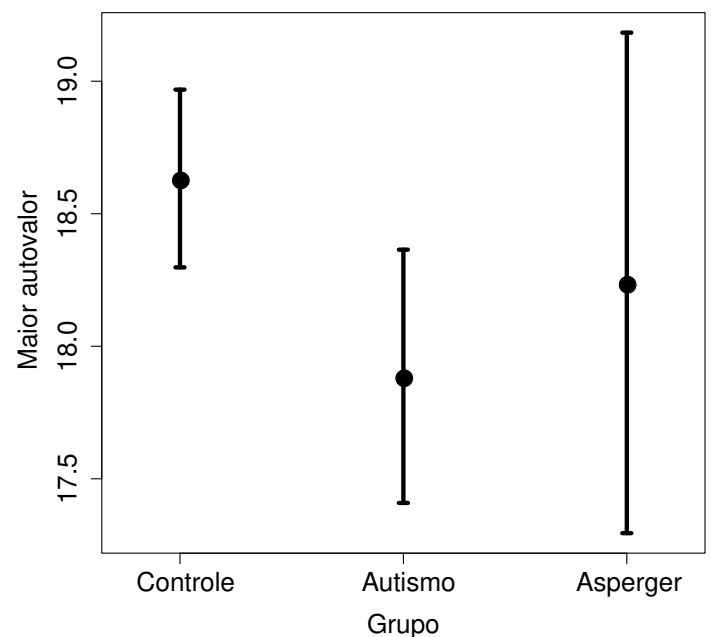

(d) A conectividade inter-hemisférica ( $\mathrm{CIH})$ média do córtex orbitofrontal medial.

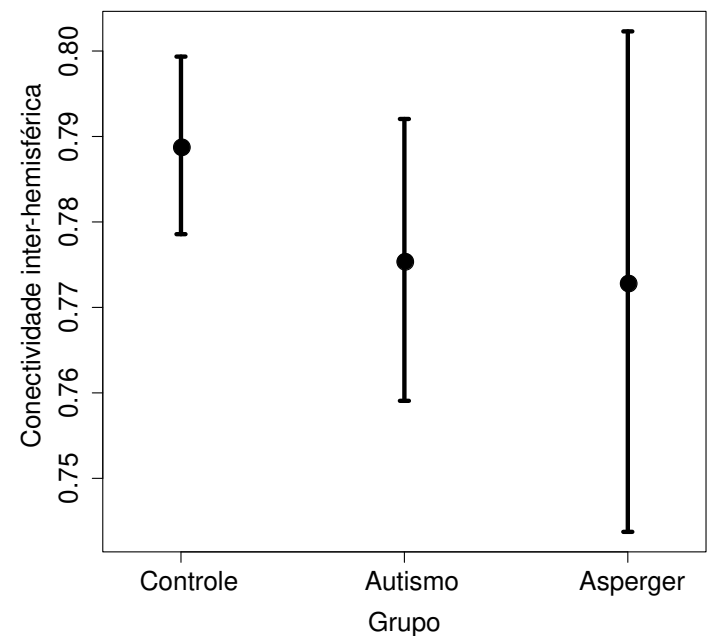

Figura 7.5: Medidas de conectividade funcional e de volumes cerebrais médias. Em cada gráfico, nós comparamos os valores médios obtidos para controles, indivíduos com autismo e indivíduos com Asperger com um intervalo de confiância de 95\% (barra de erro). (a) O volume médio do corpo caloso anterior em cada grupo ( $p$-valor $=0.428)$. (b) A média do maior autovalor da rede modo padrão é contrastada entre os grupos ( $p$-valor $=0.025)$. (c) A média do raio estimado do grafo aleatório geométrico ( $p$-valor $=0.048)$. (d) A conectividade inter-hemisférica $(C I H)$ média do córtex orbitofrontal medial ( $p$-valor $=0.230)$. 


\subsection{Discussão}

Nós interpretamos o maior autovalor como uma medida descritiva da conectividade da rede funcional do cérebro e de propagação de informação. Além disso, nós estamos interessados na relação entre o maior autovalor e parâmetros de modelos de grafo aleatório. Ao modelarmos a rede modo padrão do cérebro como um grafo aleatório geométrico, nós podemos interpretar o maior autovalor como uma medida diretamente associada ao parâmetro (raio). Esse modelo de grafo aleatório é utilizado muitas vezes para representar sistemas em que há uma correlação espaço-temporal entre os vértices, como redes de WiFi (Nekovee, 2007). Assim, nós testamos se o raio estimado do modelo geométrico está relacionado com a distância física entre os pares de ROIs. Nós ajustamos um modelo de regressão linear no qual a variável resposta é a distância média entre pares de ROIs cocnectadas e a variável explicativa é o raio do modelo geométrico. Para reduzir o efeito de movimentação na cabeça nós adicionamos a medida de deslocamento de quadros (FD) como covariável. De fato, nós observamos que o raio estimado está correlacionado positivamente com a distância física média entre as ROIs conectadas (coeficiente da regressão $=0.741$, p-valor < 0.001), mas não está significativamente correlacionado com o FD (coeficiente da regressão $=-0.024, \mathrm{p}$-valor $=0.341$ ). É válido notar que a informação sobre a distância física entre as ROIs não foi utilizada para construir a rede modo padrão.

Como os resultados obtidos para o raio do grafo geométrico são similares aos obtidos para o maior autovalor e ambas medidas estão correlacionadas, nós podemos concluir que à medida que o maior autovalor da rede modo padrão aumenta, o raio estimado do modelo geométrico cresce. Além disso, ROIs distantes se tornam mais conectadas, melhorando a propagação de informação dentro da rede funcional do cérebro. Consequentemente, na síndrome de Asperger, regiões distantes da rede modo padrão se tornam mais conectadas à medida que o volume da região anterior do corpo caloso aumenta. Essa associação é significativamente maior em Asperger do que nos grupos autista e de controle.

Uma característica observada em alguns casos de TEA é um volume reduzido do corpo caloso, particularmente na parte anterior (HARDAN et al., 2000; C. N. VIDAL et al., 2006). Evidências a partir de indivíduos com agenesia do corpo caloso indicam que a maior estrutura de matéria branca do cérebro está envolvida em habilidades sociais e outros processos cognitivos que requerem a integração de informações complexas (TRAVERs et al., 2015; PAUl et al., 2007).

Anormalidades no corpo caloso podem implicar em conectividade funcional anormal. Em particular a conectividade funcional da rede modo padrão tem sido consistentemente reportada como alterada em TEA (Assaf et al., 2010; Kennedy e Courchesne, 2008; Kennedy, Redcay et al., 2006; Monk et al., 2009; Weng et al., 2010; Hagen et al., 2013; Jung et al., 2014). De fato, no nosso conjunto de dados, nós observamos que no grupo autista, a conectividade (medida pelo maior autovalor) média da rede modo padrão é significativamente menor.

Portanto, o volume do corpo caloso (região anterior) e a rede modo padrão (maior autovalor) desempenham um papel importante no processo cognitivo social em TEA. Além de estudarmos a relação entre a rede modo padrão e o volume do corpo caloso, nós investigamos se a conectividade funcional de regiões homotópicas (regiões do cérebro 
geometricamente simétricas) dessa rede está associada ao volume da parte anterior do corpo caloso. Nossos resultados sugerem que a conectividade funcional entre o córtex orbitofrontal medial esquerdo e direito tem uma associação aumentada com o volume do corpo caloso anterior em Asperger, quando comparados com controles e o grupo autista.

Corpo caloso reduzido, especialmente na região anterior, e menos conexões interhemisféricas na parte frontal do cérebro em TEA foram reportadas por SCHAER et al., 2013. Os autores também observaram que menos conexões inter-hemisféricas frontais correlacionam com a severidade de comportamento restrito e repetitivo. Mais tarde, RolAnd et al., 2017 confirmaram o papel do corpo caloso na conectividade funcional entre os hemisférios esquerdo e direito. Eles observaram que após a calosotomia, os indivíduos operados apresentaram conectividade funcional inter-hemisférica próxima de zero na região frontal e parietal, mas ainda apresentaram conectividade funcional inter-hemisférica residual nas redes sensoriomotora e visual (RolAnd et al., 2017). Esses resultados são consistentes com as relações encontradas no nosso conjunto de dados entre a conectividade das regiões inter-hemisféricas do córtex orbitofrontal medial (sub-regiões do lobo frontal) e o volume do corpo caloso anterior em Asperger.

O córtex orbitofrontal medial é parte do córtex pré-frontal, que está envolvido em sintomas centrais do TEA, como a regulação de respostas socio-emocionais e teoria da mente (AJRAm et al., 2017), e alterações da atividade pré-frontal (GOLDBERG et al., 2011) e da conectividade (AJRAM et al., 2017).

A relação entre o córtex pré-frontal e o corpo caloso já foi estuda por OzALAY et al., 2013. Os autores defendem a ideia de que o desenvolvimento do corpo caloso e o córtex pré-frontal se influenciam mutuamente. Eles mostraram que o tamanho do corpo caloso anterior está relacionado com o volume do córtex pré-frontal em controles . Essa associação parece estar alterada em pacientes com depressão que não utilizam drogas.

O córtex orbitofrontal é conectado a estruturas neuroanatômicas diretamente envolvidas no processamento emocional e executivo (como cíngulo anterior, hipotálamo, formação hipocampal, amígdala, corpo estriado ventral e áreas temporais mediais (JАскоwsKi et al., 2012). Ele também está associado à tomada de decisão e ao comportamento adaptativo em reação a acontecimentos inesperados (JAcкоWski et al., 2012). Alguns estudos também associam lesões orbitofrontais a desinibição socioemocional e disfunções executivas (JACKOWsKi et al., 2012). Além disso, estudos a partir de dados de neuroimagem estruturais e funcionais indicam que o córtex orbitofrontal desempenha um papel importante em diversos transtornos psiquiátricos (JАСКоWsкi et al., 2012).

Em particular, alterações no córtex orbitofrontal foram associadas com autismo (BACHEVALIER e LOVELAND, 2006). Girgis et al., 2007 encontraram matéria cinzenta reduzida no córtex orbitofrontal de crianças diagnosticadas com autismo. Eles também observaram uma correlação entre estruturas de matéria branca do córtex orbitofrontal e comprometimento das interações sociais. Portanto, a associação entre a conectividade orbitofrontal medial e o corpo caloso está de acordo com a ideia de que ambas as estruturas desempenham um papel importante nos prejuízos sociais observados em Asperger.

O fato de a associação entre a rede modo padrão do cérebro e o volume do corpo caloso 
anterior ser maior em Asperger em comparação com o grupo autista sugere que o conjunto de estruturas alteradas que regulam a conectividade funcional não é exatamente o mesmo em ambos grupos. Estudos anteriores reportaram mais estruturas alteradas em autismo do que em Asperger (Jou et al., 2010; G. M. McAlonAn et al., 2009).

É natural esperar que controles sejam mais similares a Asperger (cujos sintomas são mais brandos) do que o grupo autista. Contudo, similarmente ao autismo, no grupo controle o maior autovalor da rede modo padrão não está significativamente associado com o volume do corpo caloso anterior. Ao compararmos os coeficientes dos modelos lineares com um teste $z$, nós observamos que a associação maior em Asperger em comparação aos grupos autista e de controle não é devida a diferenças de tamanho amostral/poder estatístico. Uma possível explicação para a associação ser menor nos controles é o fato de que, nesses indivíduos, as variações no corpo caloso não resultam em conectividade anormal (possivelmente devido a estruturas compensatórias). Além disso, diferentes variabilidades intra-grupos podem influenciar os resultados.

ANDERSON et al., 2011 reportaram que, apesar do volume do corpo caloso e a conectividade interhemisférica estarem reduzidas no grupo autista, não foi detectada relação direta significativa entre as duas medidas em autismo nem em controles. Os autores sugerem que outras estruturas reguladoras da conectividade funcional podem compensar as variações no tamanho do corpo caloso. Esses resultados são consistentes com nossas análises, como a associação entre o maior autovalor da rede modo padrão / conectividade interhemisférica e o volume da região anterior do corpo caloso não é significativa no grupo autista nem em controles.

Como a movimentação da cabeça é uma questão crítica em estudos de fMRI, nós realizamos os seguintes passos: (i) rotacionamos e deslocamos os dados do volume do cérebro em cada quadro discrepante; (ii) realizamos o processo de scrubbing (Power et al., 2012), removendo os quadros com maior movimentação; (iii) incluímos uma medida de descolamento de quadros (FD) como covariável em todas as análises envolvendo os dados de fMRI; e (iv) comparamos os resultados antes e após o scrubbing. No passo (iv), nós observamos que os resultados antes e após o scrubbing são similares (Tabelas B.1 e B.2 no Apêndice B). Finalmente, nós comparamos o FD médio entre controle, autismo e Asperger, e não encontramos diferença significativa entre os grupos ( $p$-valor ANOVA $=0.067$ ).

Uma limitação deste estudo é o fato de o tamanho amostral não ser grande o suficiente para realizar todos as análises separadamente por laboratório e/ou por idade. Dados sobre a destreza manual para todos indivíduos permitiriam uma investigação dos seus efeitos sobre a conectividade funcional entre os dois hemisférios em TEA (CHERBUIN e BRINKMAN, 2006; KoedA, 2017; KnAus et al., 2016). Além disso, dados comportamentais e cognitivos para um grupo maior de indivíduos poderia permitir a investigação de mecanismos envolvidos em diferentes sintomas de TEA (LoRD et al., 2012). Direções futuras dessa pesquisa incluem o estudo da conectividade funcional em diferentes condições, como fMRI baseado em tarefas, envolvendo a ativação de diferentes áreas do cérebro. 


\subsection{Conclusões}

Na síndrome de Asperger, há uma associação aumentada entre a rede modo padrão e o volume da região anterior do corpo caloso em comparação com autismo e controles. Além disso, a associação da conectividade entre o córtex orbitofrontal medial esquerdo e direito (parte da rede modo padrão) com o volume do corpo caloso anterior é maior em Asperger. Assim, nossos resultados sugerem que diferentes mecanismos estão envolvidos na regulação da conectividade funcional em Asperger e autismo. Ao representar a rede modo padrão pelo maior autovalor da matriz de adjacência podemos capturar a relação com o parâmetro do modelo de grafo aleatório. A partir do ajuste do modelo geométrico à rede modo padrão e da relação de seu parâmetro (raio) com a distância física entre ROIs conectadas, observamos que, à medida que o volume do corpo caloso anterior cresce, ROIs distantes tornam-se mais conectadas. O maior autovalor descreve, portanto, características chave das redes funcionais do cérebro e pode ajudar na investigação de como a organização da rede muda com estruturas cerebrais. 


\section{Capítulo 8}

\section{Considerações finais}

Neste trabalho representamos redes do mundo real (muitas vezes chamadas de "complexas") como grafos aleatórios. Uma vez conhecido o modelo gerador de um grafo, podemos descrevê-lo por um conjunto de parâmetros. Assim, neste trabalho, nos focamos em dois problemas: (i) estimar o parâmetro de modelos de grafo aleatório e (ii) medir a relação entre parâmetros de grafos aleatórios e variáveis aleatórias. Tanto para (i) quanto para (ii), estudamos abordagens baseadas nos autovalores das matrizes de adjacência dos grafos.

Nossa principal contribuição para o problema (i) foi derivar a consistência de estimadores de parâmetro baseados na norma $\ell_{1}$ entre densidades espectrais e entre distribuições acumuladas, que são adaptações do procedimento proposto por TAKAHASHI et al., 2012. Uma vantagem em se trabalhar com a densidade espectral ou com a distribuição acumulada dos autovalores é que elas podem ser estimadas empiricamente para qualquer grafo não dirigido e descrevem (teórica e empiricamente) diversos modelos. $\mathrm{Na}$ abordagem proposta por TAKAHASHI et al., 2012 para estimação de parâmetro, para cada modelo de grafo aleatório, conseguimos construir um procedimento para estimar o parâmetro. Essa generalidade é particularmente útil para o problema de seleção de modelos, em que utilizamos uma medida de ajuste que seja aplicável a todos modelos considerados.

É importante notar que, apesar do procedimento poder ser aplicado a qualquer modelo que gera grafos aleatórios, os resultados teóricos a respeito da consistência do estimador de parâmetro foram verificados apenas para poucos modelos aleatórios cujos limites da densidade espectral existem e são obtidos analiticamente (Erdős-Rényi, $d$-regular, modelo bloco estocástico). Não obstante, em experimentos de simulação, em modelos cujas densidades espectrais assintóticas não são conhecidas analiticamente, como o geométrico, Watts-Strogatz e Barabási-Albert, o estimador de parâmetro aproxima-se do valor esperado à medida que o tamanho do grafo aumenta.

Para o problema (ii), ao invés de estimarmos os parâmetros diretamente, podemos trabalhar com uma característica do grafo que é uma função de parâmetros de diversos modelos de grafo aleatório: o maior autovalor da matriz de adjacência.

Nossa principal contribuição para o problema (ii) foi estudar a relação entre a rede modo padrão do cérebro e o volume de sub-regiões do corpo caloso no Transtorno do Espectro 
Autista, ilustrando uma aplicação de análise estatística de grafos aleatórios baseada no espectro. A relação foi analisada de duas maneiras: uma a partir da seleção de modelos e estimação de parâmetros e outra a partir do maior autovalor do grafo. Ambas foram capazes de capturar a mesma relação entre a rede modo padrão e o volume do corpo caloso anterior, que é aumentada em Asperger em comparação com autismo e controles. Como o corpo caloso liga os hemisférios esquerdo e direito do cérebro, também investigamos a relação da conectividade de regiões simétricas entre hemisférios e que fazem parte da rede modo padrão com o volume do corpo caloso anterior. Novamente, notamos uma relação aumentada em Asperger. Nossos resultados sugerem que as bases neurais de Asperger e autismo não são exatamente as mesmas, uma vez que a rede modo padrão de ambos parece ser afetada por diferentes mecanismos.

Por fim, participamos do desenvolvimento de um pacote do R chamado statGraph para análise de grafos e o disponibilizamos no CRAN (https://CRAN.R-project.org/package= statGraph). Os métodos para a estimação de parâmetros e para selação de modelos correspondem às funções graph. param.estimator e graph.model. selection, respectivamente. Já o método para testar a correlação entre grafos a partir do maior autovalor está implementado na função graph. cor. test. Outros métodos disponíveis incluem um método para agrupamento de grafos e testes estatísticos para verificar se grafos ou conjuntos de grafos foram gerados pelo mesmo processo aleatório.

Possíveis extensões das abordagens apresentadas incluem estudo da convergência de densidades espectrais de outros modelos de grafo aleatório ampliando o escopo dos resultados teóricos sobre a consistência do estimador, estudo da distribuição do estimador de parâmetro, estimação de parâmetro de modelos de grafos dirigidos (cujo espectro pode apresentar parte imaginária não nula), estudo da relação entre a rede modo padrão do cérebro e variáveis comportamentais e investigação de outras estruturas cerebrais envolvidas em Asperger e autismo. 


\section{Apêndice A}

\section{Coeficientes estimados nos controles, indivíduos com autismo e Asperger}

Tabela A.1: Descrição dos parâmetros obtidos a partir do modelo linear utilizado para analisar a associação entre o volume do corpo caloso anterior (CC anterior) e o maior autovalor da rede modo padrão em autismo.

\begin{tabular}{ccccc}
\hline Variável & Valor estimado & Erro padrão & t-valor & p-valor \\
\hline Intercepto & 0.408 & 0.506 & 0.805 & 0.422 \\
Sítio: CMU & 0.274 & 0.663 & 0.413 & 0.680 \\
Sítio: KKI & -1.118 & 0.684 & -1.634 & 0.104 \\
Sítio: Leuven_1 & -0.089 & 0.550 & -0.163 & 0.871 \\
Sítio: Leuven_2 & 0.225 & 0.570 & 0.394 & 0.694 \\
Sítio: MaxMun & 0.545 & 1.067 & 0.511 & 0.610 \\
Sítio: NYU & -0.412 & 0.518 & -0.794 & 0.428 \\
Sítio: Pitt & 0.256 & 0.545 & 0.470 & 0.639 \\
Sítio: SBL & -0.374 & 1.084 & -0.345 & 0.731 \\
Sítio: SDSU & 1.487 & 1.062 & 1.400 & 0.163 \\
Sítio: Trinity & 0.601 & 0.599 & 1.004 & 0.317 \\
Sítio: UCLA_1 & -0.450 & 0.533 & -0.844 & 0.400 \\
Sítio: UCLA_2 & 0.301 & 0.831 & 0.362 & 0.718 \\
Sítio: UM_1 & -0.724 & 0.535 & -1.353 & 0.177 \\
Sítio: UM_2 & -0.915 & 0.568 & -1.612 & 0.109 \\
Sítio: USM & -0.888 & 0.506 & -1.755 & 0.081 \\
Sítio: Yale & 0.263 & 0.687 & 0.383 & 0.702 \\
Idade & 0.003 & 0.081 & 0.035 & 0.972 \\
Sexo masculino & -0.065 & 0.216 & -0.300 & 0.764 \\
FD & -0.100 & 0.075 & -1.335 & 0.183 \\
Volume intracraniano & 0.082 & 0.086 & 0.956 & 0.340 \\
Volume do CC anterior & -0.094 & 0.074 & -1.274 & 0.204 \\
\hline
\end{tabular}


Tabela A.2: Descrição dos parâmetros obtidos a partir do modelo linear utilizado para analisar a associação entre o volume do corpo caloso anterior (CC anterior) e o maior autovalor da rede modo padrão na sindrome de Asperger.

\begin{tabular}{c|cccc} 
Variável & Valor estimado & Erro padrão & t-valor & p-valor \\
\hline Intercepto & -1.046 & 0.519 & -2.013 & 0.049 \\
Sítio: MaxMun & 0.936 & 0.633 & 1.480 & 0.144 \\
Sítio: NYU & 0.888 & 0.635 & 1.397 & 0.168 \\
Sítio: SBL & 0.532 & 0.876 & 0.608 & 0.546 \\
Sítio: SDSU & 0.713 & 0.656 & 1.088 & 0.281 \\
Sítio: Trinity & 1.386 & 0.758 & 1.828 & 0.073 \\
Sítio: UM_1 & 0.541 & 0.613 & 0.883 & 0.381 \\
Sítio: UM_2 & -0.086 & 0.702 & -0.122 & 0.903 \\
Sítio: Yale & 1.302 & 0.662 & 1.966 & 0.054 \\
Idade & -0.259 & 0.177 & -1.466 & 0.148 \\
Sexo masculino & 0.298 & 0.384 & 0.777 & 0.441 \\
FD & -0.121 & 0.140 & -0.861 & 0.393 \\
Volume intracraniano & 0.072 & 0.182 & 0.392 & 0.696 \\
Volume CC anterior & 0.341 & 0.133 & 2.558 & 0.013
\end{tabular}


Tabela A.3: Descrição dos parâmetros obtidos a partir do modelo linear utilizado para analisar a associação entre o volume do corpo caloso anterior (CC anterior) e o maior autovalor da rede modo padrão nos controles.

\begin{tabular}{c|cccc} 
Variável & Valor estimado & Erro padrão & t-valor & p-valor \\
\hline Intercepto & -0.719 & 0.310 & -2.316 & 0.021 \\
Sítio: CMU & 1.150 & 0.535 & 2.151 & 0.032 \\
Sítio: KKI & 0.255 & 0.384 & 0.665 & 0.506 \\
Sítio: Leuven_1 & 0.905 & 0.390 & 2.321 & 0.021 \\
Sítio: Leuven_2 & 1.069 & 0.390 & 2.739 & 0.006 \\
Sítio: MaxMun & 0.658 & 0.350 & 1.880 & 0.061 \\
Sítio: NYU & 0.825 & 0.330 & 2.503 & 0.013 \\
Sítio: Olin & 1.097 & 0.392 & 2.798 & 0.005 \\
Sítio: Pitt & 1.187 & 0.370 & 3.213 & 0.001 \\
Sítio: SBL & 1.791 & 0.407 & 4.402 & $<0.001$ \\
Sítio: SDSU & 1.342 & 0.378 & 3.546 & $<0.001$ \\
Sítio: Standford & 0.053 & 0.427 & 0.123 & 0.902 \\
Sítio: Trinity & 1.718 & 0.369 & 4.652 & $<0.001$ \\
Sítio: UCLA_1 & 0.793 & 0.370 & 2.141 & 0.033 \\
Sítio: UCLA_2 & 0.904 & 0.490 & 1.844 & 0.066 \\
Sítio: UM_1 & 0.020 & 0.336 & 0.059 & 0.953 \\
Sítio: UM_2 & 0.164 & 0.365 & 0.450 & 0.653 \\
Sítio: USM & 0.464 & 0.355 & 1.305 & 0.193 \\
Sítio: Yale & 1.057 & 0.380 & 2.782 & 0.006 \\
Idade & -0.127 & 0.062 & -2.060 & 0.040 \\
Sexo masculino & -0.047 & 0.118 & -0.398 & 0.691 \\
FD & -0.127 & 0.053 & -2.398 & 0.017 \\
Volume intracraniano & 0.150 & 0.061 & 2.471 & 0.014 \\
Volume CC anterior & -0.003 & 0.051 & -0.061 & 0.951
\end{tabular}





\section{Apêndice B}

\section{Resultados obtidos antes do procedimento de scrubbing}

Para avaliar se o processo de scrubbing afetou significativamente os resultados, nós repetimos as análises descritas na Seção 7.2.5 usando o conjunto de dados sem a aplicação do procedimento de scrubbing.

Ao comparar a associação entre o maior autovalor da rede modo padrão e o volume de sub-regiões do corpo caloso (posterior, posterior médio, central, anterior médio e anterior) entre os grupo (Asperger e autismo), nós observamos na Tabela B.1 que as conclusões permanecem as mesmas com ou sem o procedimento de scrubbing. Isto é, apenas no corpo caloso anterior observamos uma associação significativamente maior em Asperger do que em autismo ( $\mathrm{p}$-valor ajustado por Bonferroni $=0.02$ ). Além disso, ao compararmos a relação do maior autovalor com o volume do corpo caloso anterior em controles, não há evidência estatística de que ela seja diferente da associação observada no grupo autista ( $\mathrm{p}$-value $=0.290)$, mas é significativamente menor em relação aos indivíduos com síndrome de Asperger $(\mathrm{p}$-value $=0.017)$.

Tabela B.1: Comparação da associação entre a rede modo padrão e o volume de sub-regiões do corpo caloso entre indivíduos com autismo e indivíduos com síndrome de Asperger a partir de dados de ressonância magnética funcional do cérebro sem aplicação do procedimento de scrubbing. Nós testamos se a associação para cada uma das sub-regiões (posterior, posterior médio, central, anterior médio e anterior) é igual em ambos grupos. Cada célula contém o p-valor nomimal e, em parênteses, o p-valor ajustado pelo método de Bonferroni. P-valores ajustados abaixo de 0.05 estão destacados em negrito.

\begin{tabular}{ccccc}
\hline & \multicolumn{3}{c}{ Corpo caloso } \\
Posterior & Posterior médio & Central & Anterior médio & Anterior \\
\hline $0.185(0.93)$ & $0.342(1.00)$ & $0.020(0.10)$ & $0.224(1.00)$ & $\mathbf{0 . 0 0 4 ( 0 . 0 2 )}$ \\
\hline
\end{tabular}

Os coeficientes do modelo linear entre o maior autovalor da rede modo padrão e o volume do corpo caloso anterior obtidos para cada grupo e os p-valores correspondentes são exibidos na Tabela B.2. 
Tabela B.2: Coeficientes e p-valores dos modelos lineares ajustados para o maior autovalor em controles, indivíduos com autismo e Asperger a partir de dados sem aplicação do procedimento de scrubbing. Na primeira linha, cada coeficiente mede a associação entre o maior autovalor da rede modeo padrão e o volume do corpo caloso anterior. Os p-valores do teste de nulidade dos coeficientes são exibidos na segunda linha. Os p-valores ajustados menores do que 0.5 estão destacados em negrito.

\begin{tabular}{c|ccc}
\hline & \multicolumn{3}{|c}{ Grupo } \\
& Controle & Autismo & Asperger \\
\hline Coeficiente & -0.011 & -0.106 & 0.333 \\
P-valor & 0.828 & 0.153 & $\mathbf{0 . 0 1 7}$ \\
\hline
\end{tabular}




\section{Referências}

[Avrachenkov et al. 2015] K. Avrachenkov, L. Cottatellucci e A. Kadavankandy. "Spectral properties of random matrices for stochastic block model". Em: 2015 13th International Symposium on Modeling and Optimization in Mobile, Ad Hoc, and Wireless Networks (WiOpt). Mai. de 2015, pgs. 537-544. DoI: 10.1109/WIOPT. 2015.7151116 (citado nas pgs. 2, 15-18).

[AJRAm et al. 2017] L. A. AjRAm et al. "Shifting brain inhibitory balance and connectivity of the prefrontal cortex of adults with autism spectrum disorder". en. Em: Translational Psychiatry 7.5 (mai. de 2017), e1137. IsSN: 2158-3188. DOI: 10.1038/tp.2017.104. URL: https://www.nature.com/articles/tp2017104 (acesso em 04/07/2018) (citado na pg. 74).

[Ambroise e Matias 2012] Christophe Ambroise e Catherine Matias. "New consistent and asymptotically normal parameter estimates for random-graph mixture models". en. Em: Fournal of the Royal Statistical Society: Series B (Statistical Methodology) 74.1 (jan. de 2012), pgs. 3-35. ISSN: 1467-9868. DOI: $10.1111 /$ j. 1467 9868.2011.01009.x. URL: http:// onlinelibrary.wiley.com/doi/10.1111/j.14679868.2011.01009.x/abstract (citado nas pgs. 2, 53).

[Allman et al. 2011] Elizabeth S. Allman, Catherine Matias e John A. Rhodes. "Parameter identifiability in a class of random graph mixture models". Em: fournal of Statistical Planning and Inference 141.5 (mai. de 2011), pgs. 1719-1736. IssN: 0378-3758. DOI: 10.1016/j.jspi.2010.11.022. URL: http://www.sciencedirect.com/ science/article/pii/S0378375810005227 (citado na pg. 2).

[ANDERSON et al. 2011] Jeffrey S. ANDERson et al. "Decreased interhemispheric functional connectivity in autism”. eng. Em: Cerebral Cortex (New York, N.Y.: 1991) 21.5 (mai. de 2011), pgs. 1134-1146. ISSN: 1460-2199. DoI: 10.1093/cercor/bhq190 (citado nas pgs. 61, 75).

[ARNOLD 1967] Ludwig ARNOLD. "On the asymptotic distribution of the eigenvalues of random matrices". Em: Fournal of Mathematical Analysis and Applications 20.2 (nov. de 1967), pgs. 262-268. ISSN: 0022-247X. DoI: 10.1016/0022-247X(67)900893. URL: http:// www. sciencedirect. com/science / article / pii / 0022247 X67900893 (acesso em 14/12/2017) (citado na pg. 13). 
[AssAF et al. 2010] Michal Assaf et al. "Abnormal functional connectivity of default mode sub-networks in autism spectrum disorder patients”. eng. Em: NeuroImage 53.1 (out. de 2010), pgs. 247-256. ISSN: 1095-9572. Dor: 10.1016/j.neuroimage.2010. 05.067 (citado nas pgs. 60, 73).

[BArabÁsi e Albert 1999] Albert-László BarabÁsi e Réka Albert. "Emergence of Scaling in Random Networks”. en. Em: Science 286.5439 (out. de 1999), pgs. 509512. ISSN: 0036-8075, 1095-9203. DOI: 10.1126/science.286.5439.509. URL: http: //www.sciencemag.org/content/286/5439/509 (citado nas pgs. 1, 2, 22, 66).

[Buckner, Andrews-Hanna et al. 2008] Randy L. Buckner, Jessica R. AndrewsHANnA e Daniel L. Schacter. "The brain's default network: anatomy, function, and relevance to disease". eng. Em: Annals of the New York Academy of Sciences 1124 (mar. de 2008), pgs. 1-38. ISSN: 0077-8923. DOI: 10.1196/annals.1440.011 (citado na pg. 61).

[Bassett e Bullmore 2006] Danielle Smith Bassett e Ed Bullmore. "Small-world brain networks". eng. Em: The Neuroscientist: A Review fournal Bringing Neurobiology, Neurology and Psychiatry 12.6 (dez. de 2006), pgs. 512-523. IssN: 1073-8584. DOI: $10.1177 / 1073858406293182$ (citado na pg. 68).

[Christian F. Beckmann et al. 2005] Christian F. Beckmann, Marilena DeLuca, Joseph T. Devlin e Stephen M. Smith. "Investigations into resting-state connectivity using independent component analysis”. eng. Em: Philos. Trans. R. Soc. Lond., B, Biol. Sci. 360.1457 (mai. de 2005), pgs. 1001-1013. IssN: 0962-8436. DOI: 10.1098/ rstb.2005.1634 (citado nas pgs. 60, 67).

[Blackwell et al. 2007] Paul Blackwell, Mark Edmondson-Jones e Jonathan JoRDAN. Spectra of adjacency matrices of random geometric graphs. University of Sheffield. Department of Probability e Statistics, 2007 (citado nas pgs. 2, 19).

[Besseling et al. 2018] René Besseling et al. "Functional network abnormalities consistent with behavioral profile in Autism Spectrum Disorder". Em: Psychiatry Research: Neuroimaging 275 (mai. de 2018), pgs. 43-48. IssN: 0925-4927. DOI: 10. 1016/j.pscychresns.2018.02.006. URL: http://www.sciencedirect.com/science/ article/pii/S0925492717302627 (acesso em 18/12/2018) (citado na pg. 60).

[BhAmidi et al. 2012] Shankar BhAmidi, Steven N. Evans e Arnab Sen. "Spectra of Large Random Trees”. en. Em: Journal of Theoretical Probability 25.3 (set. de 2012), pgs. 613-654. ISSN: 0894-9840, 1572-9230. DOI: 10.1007/s10959-011-0360-9. URL: http://link.springer.com/10.1007/s10959-011-0360-9 (acesso em 17/01/2018) (citado na pg. 23).

[BAuerschmidt et al. 2017] Roland Bauerschmidt, Antti Knowles e Horng-Tzer Yau. “Local Semicircle Law for Random Regular Graphs”. en. Em: Comm. Pure Appl. Math. 70.10 (out. de 2017), pgs. 1898-1960. ISSN: 1097-0312. DOI: 10.1002/cpa.21709. URL: http://onlinelibrary.wiley.com/doi/10.1002/cpa.21709/abstract (acesso em 17/01/2018) (citado na pg. 18). 
REFERÊNCIAS

[BAchevalier e Loveland 2006] Jocelyne Bachevalier e Katherine A. Loveland. "The orbitofrontal-amygdala circuit and self-regulation of social-emotional behavior in autism". Em: Neuroscience \& Biobehavioral Reviews 30.1 (jan. de 2006), pgs. 97-117. ISSN: 0149-7634. DOI: 10.1016/j.neubiorev.2005.07.002. URL: http: //www. sciencedirect.com/science/article / pii / S0149763405001193 (acesso em 04/07/2018) (citado na pg. 74).

[Bordenave 2008] Charles Bordenave. "Eigenvalues of Euclidean random matrices". en. Em: Random Structures \& Algorithms 33.4 (dez. de 2008), pgs. 515-532. ISSN: 1098-2418. DOI: 10.1002/rsa.20228. URL: http://onlinelibrary.wiley.com/doi/10. 1002/rsa.20228/abstract (citado nas pgs. 65, 69).

[Bullmore e Sporns 2009] Ed Bullmore e Olaf Sporns. "Complex brain networks: graph theoretical analysis of structural and functional systems". eng. Em: Nat. Rev. Neurosci. 10.3 (mar. de 2009), pgs. 186-198. IssN: 1471-0048. DOI: 10.1038/nrn2575 (citado nas pgs. 3, 60).

[Buckner, Sepulcre et al. 2009] Randy L. Buckner, Jorge Sepulcre et al. "Cortical hubs revealed by intrinsic functional connectivity: mapping, assessment of stability, and relation to Alzheimer's disease”. eng. Em: F. Neurosci. 29.6 (fev. de 2009), pgs. 1860-1873. ISSN: 1529-2401. DOI: 10.1523/JNEUROSCI.5062-08.2009 (citado na pg. 64).

[Biswal et al. 1997] B. B. Biswal, J. Van Kylen e J. S. Hyde. "Simultaneous assessment of flow and BOLD signals in resting-state functional connectivity maps". eng. Em: NMR in biomedicine 10.4-5 (ago. de 1997), pgs. 165-170. ISSN: 0952-3480 (citado na pg. 60).

[Cherbuin e Brinkman 2006] Nicolas Cherbuin e Cobie Brinkman. "Hemispheric interactions are different in left-handed individuals”. eng. Em: Neuropsychology 20.6 (nov. de 2006), pgs. 700-707. ISSN: 0894-4105. DOI: 10.1037/0894-4105.20.6.700 (citado na pg. 75).

[Chen e Calhoun 2018] Zikuan Chen e Vince Calhoun. "Effect of Spatial Smoothing on Task fMRI ICA and Functional Connectivity". Em: Front Neurosci 12 (fev. de 2018). ISSN: 1662-4548. DOI: 10.3389/fnins.2018.00015. URL: https://www.ncbi.nlm. nih.gov/pmc/articles/PMC5801305/ (acesso em 22/08/2019) (citado na pg. 64).

[CAimo e Friel 2011] Alberto CAimo e Nial Friel. "Bayesian inference for exponential random graph models”. Em: Social Networks 33.1 (jan. de 2011), pgs. 41-55. IssN: 0378-8733. DOI: 10.1016/j.socnet.2010.09.004. URL: http://www.sciencedirect.com/ science/article/pii/S0378873310000493 (citado na pg. 2).

[CAPInski e Kopp 2004] Marek CAPInski e Peter E. Kopp. Measure, Integral and Probability. en. Google-Books-ID: jdnGYuh58YUC. Springer Science \& Business Media, 2004. ISBN: 978-1-85233-781-0 (citado na pg. 7). 
[Chung e Lu 2002] Fan Chung e Linyuan Lu. "Connected Components in Random Graphs with Given Expected Degree Sequences”. en. Em: Annals of Combinatorics 6.2 (nov. de 2002), pgs. 125-145. ISSN: 0218-0006, 0219-3094. DOI: 10.1007/ PL00012580. URL: https://link.springer.com/article/10.1007/PL00012580 (acesso em 02/03/2018) (citado na pg. 21).

[CORDEs et al. 2000] D. CORDEs et al. "Mapping functionally related regions of brain with functional connectivity MR imaging”. eng. Em: AfNR Am f Neuroradiol 21.9 (out. de 2000), pgs. 1636-1644. IssN: 0195-6108 (citado na pg. 60).

[R. C. Craddock et al. 2012] R. Cameron Craddock, G. Andrew James, Paul E. Holtzheimer, Xiaoping P. Hu e Helen S. Mayberg. "A whole brain fMRI atlas generated via spatially constrained spectral clustering”. eng. Em: Human Brain Mapping 33.8 (ago. de 2012), pgs. 1914-1928. ISSN: 1097-0193. DOI: 10.1002/hbm.21333 (citado na pg. 63).

[C. CRAdDock et al. 2013] Cameron Craddock et al. "The neuro bureau preprocessing initiative: open sharing of preprocessed neuroimaging data and derivatives". Em: Frontiers in Neuroinformatics 7 (jan. de 2013). Dor: 10.3389/conf.fninf.2013.09. 00041 (citado na pg. 62).

[Custo et al.2017] Anna Custo et al. "Electroencephalographic Resting-State Networks: Source Localization of Microstates". eng. Em: Brain Connect 7.10 (2017), pgs. 671-682. ISSN: 2158-0022. DOI: 10.1089/brain.2016.0476 (citado na pg. 60).

[Damoiseaux, Rombouts et al. 2006] J. S. Damoiseaux, Serge A. R. B. Rombouts et al. "Consistent resting-state networks across healthy subjects". Em: Proc Natl Acad Sci U S A 103.37 (set. de 2006), pgs. 13848-13853. ISSN: 0027-8424. DOI: 10.1073/pnas. 0601417103. URL: https://www.ncbi.nlm.nih.gov/pmc/articles/PMC1564249/ (acesso em 26/06/2018) (citado na pg. 67).

[Damoiseaux, C. F. Beckmann et al. 2008] J. S. Damoiseaux, C. F. Beckmann et al. "Reduced resting-state brain activity in the "default network"in normal aging". eng. Em: Cereb. Cortex 18.8 (ago. de 2008), pgs. 1856-1864. ISSN: 1460-2199. DOI: 10.1093/cercor/bhm207 (citado na pg. 67).

[Dacunha-Castelle e Duflo 1986] Didier Dacunha-Castelle e Marie Duflo. Probability and statistics. Vol. II. Springer-Verlag, New York, 1986 (citado nas pgs. 4143).

[Di Martino et al. 2014] A. Di Martino et al. "The autism brain imaging data exchange: towards a large-scale evaluation of the intrinsic brain architecture in autism”. eng. Em: Molecular Psychiatry 19.6 (jun. de 2014), pgs. 659-667. IssN: 1476-5578. DOI: 10.1038/mp.2013.78 (citado nas pgs. 4, 62).

[DAvidson e Levin 2005] Eric Davidson e Michael Levin. "Gene regulatory networks". en. Em: Proceedings of the National Academy of Sciences of the United States of America 102.14 (abr. de 2005), pgs. 4935-4935. IssN: 0027-8424, 1091-6490. DOI: 
10.1073/pnas.0502024102. URL: http://www.pnas.org/content/102/14/4935 (citado na pg. 1).

[Dorogovtsev et al. 2003] Sergey N. Dorogovtsev, Alexander V. Goltsev, José FF Mendes e Alexander N. SAmukhin. "Spectra of complex networks". Em: Physical Review E 68.4 (2003), pg. 046109. URL: http://journals.aps.org/pre/abstract/10. 1103/PhysRevE.68.046109 (citado na pg. 65).

[Dumitriu e PAl 2012] Ioana Dumitriu e Soumik Pal. "Sparse regular random graphs: Spectral density and eigenvectors”. Em: The Annals of Probability 40.5 (2012), pgs. 2197-2235. ISSN: 0091-1798. DOI: 10.2307/41713963. URL: http://www.jstor. org/stable/41713963 (citado nas pgs. 2, 18).

[Duffy et al. 2013] Frank H. Duffy, Aditi Shankardass, Gloria B. McAnulty e Heidelise Als. "The relationship of Asperger's syndrome to autism: a preliminary EEG coherence study”. Em: BMC Medicine 11 (2013), pg. 175. Issn: 1741-7015. DOI: 10.1186/1741-7015-11-175. uRL: http://dx.doi.org/10.1186/1741-7015-11-175 (citado nas pgs. 59, 60).

[ERdős e RÉnyi 1959] Paul ERdős e Alfréd RÉnyi. “On random graphs”. Em: Publicationes Mathematicae Debrecen 6 (1959), pgs. 290-297 (citado nas pgs. 1, 13, 66).

[FARKas et al. 2001] Illés J. FARKAS, Imre Derényi, Albert-László BARABÁsi e Tamás VICSEK. "Spectra of "real-world" graphs: Beyond the semicircle law". en. Em: Physical Review E 64.2 (jul. de 2001). IssN: 1063-651X, 1095-3787. DOI: 10.1103/ PhysRevE.64.026704. uRL: https://link.aps.org/doi/10.1103/PhysRevE.64.026704 (acesso em 17/01/2018) (citado nas pgs. 2, 20, 22, 23).

[Fischl, Salat, Busa et al. 2002] Bruce Fischl, David H. Salat, Evelina Busa et al. "Whole brain segmentation: automated labeling of neuroanatomical structures in the human brain”. eng. Em: Neuron 33.3 (jan. de 2002), pgs. 341-355. ISSN: 0896-6273 (citado na pg. 62).

[Fischl, Salat, Kouwe et al. 2004] Bruce Fischl, David H. Salat, André J. W. van der Kouwe et al. "Sequence-independent segmentation of magnetic resonance images”. eng. Em: NeuroImage 23 Suppl 1 (2004), S69-84. ISSN: 1053-8119. DOI: 10.1016/j.neuroimage.2004.07.016 (citado na pg. 62).

[Füredi e Komlós 1981] Z. FÜredi e J. KomLós. "The eigenvalues of random symmetric matrices”. en. Em: Combinatorica 1.3 (set. de 1981), pgs. 233-241. ISSN: 0209-9683, 1439-6912. DOI: 10.1007/BF02579329. URL: http://link.springer.com/article/10. 1007/BF02579329 (citado na pg. 65).

[Fox, SNyder et al. 2005] Michael D. Fox, Abraham Z. SNyder et al. "The human brain is intrinsically organized into dynamic, anticorrelated functional networks". en. Em: PNAS 102.27 (jul. de 2005), pgs. 9673-9678. IssN: 0027-8424, 1091-6490. DOI: 10.1073/pnas.0504136102. uRL: http://www.pnas.org/content/102/27/9673 (acesso em 15/06/2018) (citado nas pgs. 60, 61). 
[Fox e Raichle 2007] Michael D. Fox e Marcus E. Raichle. "Spontaneous fluctuations in brain activity observed with functional magnetic resonance imaging”. eng. Em: Nat. Rev. Neurosci. 8.9 (set. de 2007), pgs. 700-711. IssN: 1471-003X. DOI: 10.1038/nrn2201 (citado na pg. 61).

[Fransson 2005] Peter Fransson. "Spontaneous low-frequency BOLD signal fluctuations: an fMRI investigation of the resting-state default mode of brain function hypothesis". eng. Em: Hum Brain Mapp 26.1 (set. de 2005), pgs. 15-29. IssN: 10659471. DOI: 10.1002/hbm.20113 (citado nas pgs. 60, 61).

[Friston et al. 1993] K. J. Friston, C. D. Frith, P. F. Liddle e R. S. Frackowiak. "Functional connectivity: the principal-component analysis of large (PET) data sets". eng. Em: F. Cereb. Blood Flow Metab. 13.1 (jan. de 1993), pgs. 5-14. Issn: 0271-678X. DOI: 10.1038/jcbfm.1993.4 (citado na pg. 60).

[Frank e Strauss 1986] Ove Frank e David Strauss. "Markov Graphs". Em: fournal of the American Statistical Association 81.395 (set. de 1986), pgs. 832-842. ISSN: 0162-1459. DOI: 10.1080/01621459.1986.10478342. URL: http://www.tandfonline. com/doi/abs/10.1080/01621459.1986.10478342 (citado na pg. 1).

[Fujita, Lira et al. 2019] André Fujita, Eduardo Silva Lira et al. "A semi-parametric statistical test to compare complex networks". en. Em: $\mathcal{F}$ Complex Netw (). DOI: 10.1093/comnet/cnz028. URL: https:// academic . oup.com / comnet / advance article/doi/10.1093/comnet/cnz028/5543003 (acesso em 24/10/2019) (citado na pg. 2).

[Fujita, Takahashi et al. 2017] André Fujita, Daniel Yasumasa Takahashi, Joana Bisol BALARdin, Maciel Calebe VidAl e João Ricardo SATo. "Correlation between graphs with an application to brain network analysis”. Em: Computational Statistics \& Data Analysis 109 (mai. de 2017), pgs. 76-92. ISSN: 0167-9473. DOI: 10. 1016/j.csda.2016.11.016. uRL: http://www.sciencedirect.com/science/article/pii/ S0167947316302900 (citado nas pgs. 3, 61, 65, 68).

[Fujita, M. C. Vidal et al. 2017] André Fujita, Maciel Calebe Vidal e Daniel Y TAKAHASHI. "A Statistical Method to Distinguish Functional Brain Networks". eng. Em: Frontiers in Neuroscience 11 (2017), pg. 66. ISSN: 1662-4548. DoI: 10.3389/fnins. 2017.00066 (citado na pg. 2).

[Fienberg e Wasserman 1981] Stephen E. Fienberg e Stanley S. Wasserman. "Categorical Data Analysis of Single Sociometric Relations". Em: Sociological Methodology 12 (1981), pgs. 156-192. ISSN: 0081-1750. DOI: 10.2307/270741. URL: http: //www.jstor.org/stable/270741 (citado nas pgs. 1, 15).

[GARRIty et al. 2007] Abigail G. GARRITy et al. "Aberrant "default mode"functional connectivity in schizophrenia”. eng. Em: Am J Psychiatry 164.3 (mar. de 2007), pgs. 450-457. ISSN: 0002-953X. DOI: 10.1176/ajp.2007.164.3.450 (citado nas pgs. 3 , $60)$. 
REFERÊNCIAS

[Goelman et al. 2014] Gadi Goelman, Noam Gordon e Omer Bonne. "Maximizing Negative Correlations in Resting-State Functional Connectivity MRI by TimeLag”. en. Em: PLOS ONE 9.11 (nov. de 2014), e111554. ISSN: 1932-6203. DOI: 10. 1371/journal.pone.0111554. URL: https://journals.plos.org/plosone/article?id=10. 1371/journal.pone.0111554 (acesso em 22/08/2019) (citado na pg. 64).

[Ghoshdastidar et al. 2017] Debarghya Ghoshdastidar, Maurilio Gutzeit, Alexandra CArPentier e Ulrike von Luxburg. "Two-sample Hypothesis Testing for Inhomogeneous Random Graphs”. Em: arXiv:1707.00833 [stat] (jul. de 2017). arXiv: 1707.00833. URL: http://arxiv.org/abs/1707.00833 (citado na pg. 2).

[GIRGis et al. 2007] Ragy R. Girgis et al. "Volumetric alterations of the orbitofrontal cortex in autism”. eng. Em: Prog. Neuropsychopharmacol. Biol. Psychiatry 31.1 (jan. de 2007), pgs. 41-45. IsSN: 0278-5846. DOI: 10.1016/j.pnpbp.2006.06.007 (citado na pg. 74).

[Goldberg et al. 2011] Melissa C. Goldberg et al. "Children with high functioning autism show increased prefrontal and temporal cortex activity during error monitoring”. Em: Developmental Cognitive Neuroscience 1.1 (jan. de 2011), pgs. 47-56. ISSN: 1878-9293. DOI: 10.1016/j.dcn.2010.07.002. URL: http://www.sciencedirect. com/science/article/pii/S1878929310000034 (acesso em 04/07/2018) (citado na pg. 74).

[Greicius et al. 2003] Michael D. Greicius, Ben Krasnow, Allan L. Reiss e Vinod MeNON. "Functional connectivity in the resting brain: a network analysis of the default mode hypothesis". eng. Em: Proc. Natl. Acad. Sci. U.S.A. 100.1 (jan. de 2003), pgs. 253-258. ISSN: 0027-8424. DOI: 10.1073/pnas.0135058100 (citado nas pgs. 60, 67).

[Greicius 2008] Michael D. Greicius. "Resting-state functional connectivity in neuropsychiatric disorders". eng. Em: Current Opinion in Neurology 21.4 (ago. de 2008), pgs. 424-430. ISSN: 1350-7540. DOI: 10.1097/WCO.0b013e328306f2c5 (citado nas pgs. 60, 61).

[Grenander 1963] Ulf Grenander. Probabilities on algebraic structures. en. Wiley, 1963 (citado na pg. 13).

[Gusnard et al. 2001] D. A. Gusnard, M. E. Raichle e M. E. Raichle. "Searching for a baseline: functional imaging and the resting human brain”. eng. Em: Nat. Rev. Neurosci. 2.10 (out. de 2001), pgs. 685-694. IssN: 1471-003X. DOI: 10.1038/35094500 (citado na pg. 61).

[Geyer e Thompson 1992] Charles J. Geyer e Elizabeth A. Thompson. "Constrained Monte Carlo Maximum Likelihood for Dependent Data". Em: Journal of the Royal Statistical Society. Series B (Methodological) 54.3 (1992), pgs. 657-699. ISSN: 00359246. DOI: 10.2307/2345852. URL: http://www.jstor.org/stable/2345852 (citado na pg. 2). 
[Hagen et al. 2013] Elisabeth A. H. von dem Hagen, Raliza S. Stoyanova, Simon BARON-COHEN e Andrew J. CALDER. "Reduced functional connectivity within and between 'social' resting state networks in autism spectrum conditions". eng. Em: Social Cognitive and Affective Neuroscience 8.6 (ago. de 2013), pgs. 694-701. ISSN: 1749-5024. DOI: 10.1093/scan/nss053 (citado nas pgs. 60, 73).

[Hampson et al. 2002] Michelle Hampson, Bradley S. Peterson, Pawel Skudlarski, James C. Gatenby e John C. Gore. "Detection of functional connectivity using temporal correlations in MR images". eng. Em: Hum Brain Mapp 15.4 (abr. de 2002), pgs. 247-262. ISSN: 1065-9471 (citado na pg. 60).

[Harrison et al. 2007] Ben J. Harrison, Murat Yücel, Jesus Pujol e Christos Pantelis. "Task-induced deactivation of midline cortical regions in schizophrenia assessed with fMRI”. eng. Em: Schizophr. Res. 91.1-3 (mar. de 2007), pgs. 82-86. Issn: 09209964. DOI: 10.1016/j.schres.2006.12.027 (citado nas pgs. 3, 60).

[Hamidouche et al. 2019] Mounia Hamidouche, Laura Cottatellucci e Konstantin Avrachenkov. "Spectral Analysis of the Adjacency Matrix of Random Geometric Graphs”. Em: 2019 57th Annual Allerton Conference on Communication, Control, and Computing (Allerton). ISSN: null. Set. de 2019, pgs. 208-214. DOI: 10.1109/ ALLERTON.2019.8919798 (citado nas pgs. 2, 19).

[Heuvel, René Mandl et al. 2008] Martijn P. van den Heuvel, René Mandl, Judith Luigjes e Hilleke Hulshoff Pol. "Microstructural organization of the cingulum tract and the level of default mode functional connectivity". eng. Em: $\mathcal{7}$. Neurosci. 28.43 (out. de 2008), pgs. 10844-10851. ISSN: 1529-2401. DOI: 10.1523/JNEUROSCI. 2964-08.2008 (citado na pg. 67).

[Heuvel e Hulshoff Pol 2010] Martijn P. van den Heuvel e Hilleke E. Hulshoff PoL. "Exploring the brain network: A review on resting-state fMRI functional connectivity”. Em: European Neuropsychopharmacology 20.8 (2010), pgs. 519-534. ISSN: 0924-977X. DOI: $10.1016 / \mathrm{j}$. euroneuro. 2010.03.008. URL: http://www. sciencedirect. com / science / article / pii / S0924977X10000684 (citado nas pgs. 1, $3,60,61,66)$.

[Holland et al. 1983] Paul W. Holland, Kathryn Blackmond Laskey e Samuel LeiNHARDT. "Stochastic blockmodels: First steps". Em: Social networks 5.2 (1983), pgs. 109-137. URL: http : / / www . sciencedirect . com / science / article / pii / 0378873383900217 (citado nas pgs. 1, 15).

[Heuvel, Rene Mandl et al. 2008] Martijn P. van den Heuvel, Rene Mandl e Hilleke Hulshoff Pol. "Normalized cut group clustering of resting-state FMRI data". eng. Em: PLoS ONE 3.4 (abr. de 2008), e2001. ISSN: 1932-6203. DOI: 10.1371/journal. pone.0002001 (citado na pg. 67).

[HARdan et al. 2000] A. Y. Hardan, N. J. Minshew e M. S. Keshavan. "Corpus callosum size in autism”. eng. Em: Neurology 55.7 (out. de 2000), pgs. 1033-1036. ISSN: 0028-3878 (citado nas pgs. 61, 73). 
REFERÊNCIAS

[Jackowski et al. 2012] Andrea Parolin Jаскоwsкi et al. "The involvement of the orbitofrontal cortex in psychiatric disorders: an update of neuroimaging findings". Em: Revista Brasileira de Psiquiatria 34.2 (jun. de 2012), pgs. 207-212. Issn: 1516-4446. DOI: 10.1590/S1516-44462012000200014. URL: http://www.scielo.br/scielo.php? script=sci_abstract\&pid=S1516-44462012000200014\&lng=en\&nrm=iso\&tlng=en (acesso em 04/07/2018) (citado na pg. 74).

[JiAo et al. 2011] Y. JiAo et al. "Predictive models for subtypes of autism spectrum disorder based on single-nucleotide polymorphisms and magnetic resonance imaging”. eng. Em: Advances in Medical Sciences 56.2 (2011), pgs. 334-342. IssN: 1898-4002. DOI: 10.2478/v10039-011-0042-y (citado nas pgs. 59, 60).

[Jin et al. 2015] Yan Jin et al. "Identification of infants at high-risk for autism spectrum disorder using multiparameter multiscale white matter connectivity networks". eng. Em: Hum Brain Mapp 36.12 (dez. de 2015), pgs. 4880-4896. IssN: 1097-0193. DOI: 10.1002/hbm.22957 (citado na pg. 59).

[Jou et al. 2010] Roger J. Jou, Nancy J. Minshew, Matcheri S. Keshavan e Antonio Y. HARDAN. "Cortical gyrification in autistic and Asperger disorders: a preliminary magnetic resonance imaging study”. ENG. Em: fournal of Child Neurology 25.12 (dez. de 2010), pgs. 1462-1467. ISSN: 1708-8283. DOI: 10.1177/0883073810368311 (citado nas pgs. 59, 60, 75).

[Jung et al. 2014] Minyoung Jung et al. "Default mode network in young male adults with autism spectrum disorder: relationship with autism spectrum traits". Em: Molecular Autism 5 (2014), pg. 35. IsSN: 2040-2392. DOI: 10.1186/2040-2392-5-35. URL: http://dx.doi.org/10.1186/2040-2392-5-35 (citado nas pgs. 60, 73).

[Just et al. 2007] Marcel Adam Just, Vladimir L. Cherkassky, Timothy A. Keller, Rajesh K. KanA e Nancy J. Minshew. "Functional and Anatomical Cortical Underconnectivity in Autism: Evidence from an fMRI Study of an Executive Function Task and Corpus Callosum Morphometry”. en. Em: Cerebral Cortex 17.4 (abr. de 2007), pgs. 951-961. ISSN: 1047-3211, 1460-2199. DOI: 10.1093/cercor/bhl006. URL: http://cercor.oxfordjournals.org/content/17/4/951 (citado na pg. 3).

[Kennedy e Courchesne 2008] Daniel P. Kennedy e Eric Courchesne. "Functional abnormalities of the default network during self- and other-reflection in autism". Em: Social cognitive and affective neuroscience 3.2 (jun. de 2008), pgs. 177-190. ISSN: 1749-5016. DOI: 10.1093/scan/nsn011. URL: http://www.ncbi.nlm.nih.gov/ pmc/articles/PMC2555458/ (citado nas pgs. 60, 73).

[Knaus et al. 2016] Tracey A. Knaus, Jodi Kamps e Anne L. Foundas. "Handedness in Children With Autism Spectrum Disorder”. eng. Em: Percept Mot Skills 122.2 (abr. de 2016), pgs. 542-559. ISSN: 1558-688X. DOI: 10.1177/0031512516637021 (citado na pg. 75).

[Klin 2006] Ami Klin. “Autism and Asperger syndrome: an overview”. Em: Revista Brasileira de Psiquiatria 28 (mai. de 2006), s3-s11. ISSN: 1516-4446. DOI: 10.1590/ 
S1516 - 44462006000500002. URL: http://www.scielo.br/scielo.php? script =sci abstract\&pid=S1516-44462006000500002\&Ing=en\&nrm=iso\&tlng=en (citado na pg. 59).

[Koeda 2017] Michihiko KoedA. "Default mode network and Language-related resting state functional connectivity on handedness and family history”. en. Em: (jan. de 2017). DOI: 10.4172/2168-975X.C1.010. URL: https://www.omicsonline. org / proceedings / default-mode-network-and-languagerelated-resting - statefunctional-connectivity-on-handedness-and-family-history-60258.html (acesso em 20/08/2018) (citado na pg. 75).

[Kennedy, Redcay et al. 2006] Daniel P. Kennedy, Elizabeth Redcay e Eric CourCHESNE. "Failing to deactivate: resting functional abnormalities in autism". eng. Em: Proceedings of the National Academy of Sciences of the United States of America 103.21 (mai. de 2006), pgs. 8275-8280. ISSN: 0027-8424. DOI: 10.1073/pnas. 0600674103 (citado nas pgs. 60, 73).

[Kuchaiev et al. 2009] Oleksii Kuchaiev, Po T. Wang, Zoran Nenadic e Natasa PrzULJ. "Structure of brain functional networks". eng. Em: Conference proceedings: ... Annual International Conference of the IEEE Engineering in Medicine and Biology Society. IEEE Engineering in Medicine and Biology Society. Annual Conference 2009 (2009), pgs. 4166-4170. ISSN: 1557-170X. DOI: 10.1109/IEMBS.2009.5333938 (citado na pg. 68).

[Kwon et al. 2004] Hower Kwon, Andrew W. Ow, Kate E. Pedatella, Linda J. LotsPEICH e Allan L. REISS. "Voxel-based morphometry elucidates structural neuroanatomy of high-functioning autism and Asperger syndrome”. eng. Em: Developmental Medicine and Child Neurology 46.11 (nov. de 2004), pgs. 760-764. IssN: 0012-1622 (citado nas pgs. 59, 60).

[Lincoln et al. 1998] Alan Lincoln, Eric Courchesne, Mark Allen, Ellen Hanson e Michaela EnE. "Neurobiology of Asperger Syndrome”. en. Em: Asperger Syndrome or High-Functioning Autism? Ed. por Eric Schoplen, Gary B. Mesibov e Linda J. KuncE. Current Issues in Autism. DOI: 10.1007/978-1-4615-5369-4_8. Springer US, 1998, pgs. 145-163. ISBN: 978-1-4613-7450-3 978-1-4615-5369-4. URL: http: //link.springer.com/chapter/10.1007/978-1-4615-5369-4_8 (citado nas pgs. 59, 60).

[LORD et al. 2012] Catherine Lond et al. "A multisite study of the clinical diagnosis of different autism spectrum disorders”. eng. Em: Arch. Gen. Psychiatry 69.3 (mar. de 2012), pgs. 306-313. ISSN: 1538-3636. DOI: 10.1001/archgenpsychiatry.2011.148 (citado na pg. 75).

[Lowe et al. 2008] Mark J. Lowe et al. "Resting state sensorimotor functional connectivity in multiple sclerosis inversely correlates with transcallosal motor pathway transverse diffusivity”. eng. Em: Hum Brain Mapp 29.7 (jul. de 2008), pgs. 818-827. ISSN: 1097-0193. DOI: 10.1002/hbm.20576 (citado nas pgs. 3, 60). 
REFERÊNCIAS

[M. F. MAson et al. 2007] Malia F. MAson et al. "Wandering minds: the default network and stimulus-independent thought”. eng. Em: Science 315.5810 (jan. de 2007), pgs. 393-395. ISSN: 1095-9203. DOI: 10.1126/science.1131295 (citado na pg. 61).

[R. A. Mason et al. 2008] Robert A. MAson, Diane L. Williams, Rajesh K. KanA, Nancy Minshew e Marcel Adam Just. "Theory of Mind disruption and recruitment of the right hemisphere during narrative comprehension in autism". Em: Neuropsychologia 46.1 (2008), pgs. 269-280. ISSN: 0028-3932. DOI: 10.1016/j.neuropsychologia. 2007 . 07 .018. uRL: http : / / www . sciencedirect . com / science / article / pii / S0028393207002631 (citado na pg. 3).

[Gráinne M. McAlonan et al. 2008] Gráinne M. McAlonan et al. "Distinct patterns of grey matter abnormality in high-functioning autism and Asperger's syndrome". eng. Em: $\mathcal{F}$ Child Psychol Psychiatry 49.12 (dez. de 2008), pgs. 1287-1295. IsSN: 1469-7610. DOI: 10.1111/j.1469-7610.2008.01933.x (citado na pg. 59).

[G. M. McAlonan et al. 2009] G. M. McAlonan et al. "Differential effects on whitematter systems in high-functioning autism and Asperger's syndrome". eng. Em: Psychol Med 39.11 (nov. de 2009), pgs. 1885-1893. ISSN: 1469-8978. DOI: 10.1017/ S0033291709005728 (citado nas pgs. 59, 75).

[McKay 1981] Brendan D. McKay. "The expected eigenvalue distribution of a large regular graph". Em: Linear Algebra and its Applications 40 (out. de 1981), pgs. 203216. ISSN: 0024-3795. DOI: $10.1016 / 0024-3795(81$ ) 90150 - 6. URL: http://www. sciencedirect.com/science/article/pii/0024379581901506 (citado nas pgs. 2, 18).

[Mueller et al. 2013] Laurin A. J. Mueller, Matthias Dehmer e Frank Emmert-Streib. "Comparing Biological Networks: A Survey on Graph Classifying Techniques". en. Em: Systems Biology. DOI: 10.1007/978-94-007-6803-1_2. Springer, Dordrecht, 2013, pgs. 43-63. ISBN: 978-94-007-6802-4 978-94-007-6803-1. URL: https://link. springer.com/chapter/10.1007/978-94-007-6803-1_2 (citado na pg. 1).

[Micheloyannis et al. 2006] Sifis Micheloyannis et al. "Using graph theoretical analysis of multi channel EEG to evaluate the neural efficiency hypothesis". eng. Em: Neuroscience Letters 402.3 (jul. de 2006), pgs. 273-277. ISSN: 0304-3940. DOI: 10. 1016/j.neulet.2006.04.006 (citado na pg. 68).

[Moнammadi et al. 2009] Bahram Mohammadi et al. "Changes of resting state brain networks in amyotrophic lateral sclerosis”. eng. Em: Exp. Neurol. 217.1 (mai. de 2009), pgs. 147-153. ISSN: 1090-2430. DOI: 10.1016/j.expneurol.2009.01.025 (citado nas pgs. 3,60$)$.

[Monk et al. 2009] Christopher S. Monk et al. "Abnormalities of intrinsic functional connectivity in autism spectrum disorders". eng. Em: NeuroImage 47.2 (ago. de 2009), pgs. 764-772. ISSN: 1095-9572. DOI: 10.1016/j. neuroimage.2009.04.069 (citado nas pgs. 60, 73). 
[Nekovee 2007] Maziar Nekovee. "Worm epidemics in wireless ad hoc networks". en. Em: New fournal of Physics 9.6 (2007), pg. 189. ISSN: 1367-2630. DOI: 10.1088/13672630/9/6/189. URL: http://stacks.iop.org/1367-2630/9/i=6/a=189 (citado na pg. 73).

[Neyman e Pearson 1928] J. Neyman e E. S. Pearson. "On the Use and Interpretation of Certain Test Criteria for Purposes of Statistical Inference: Part I”. Em: Biometrika 20A.1/2 (1928), pgs. 175-240. ISSN: 0006-3444. DOI: 10.2307/2331945. URL: https: //www.jstor.org/stable/2331945 (acesso em 05/12/2018) (citado na pg. 66).

[NApoli et al. 2016] Edoardo Di NApoli, Eric Polizzi e Yousef SAAd. "Efficient estimation of eigenvalue counts in an interval". en. Em: Numerical Linear Algebra with Applications 23.4 (2016), pgs. 674-692. ISSN: 1099-1506. DOI: 10.1002/nla.2048. URL: https://onlinelibrary.wiley.com/doi/abs/10.1002/nla.2048 (acesso em 22/03/2019) (citado na pg. 53).

[Newman et al. 2019] M. E. J. Newman, Xiao Zhang e Raj Rao Nadakuditi. "Spectra of random networks with arbitrary degrees". Em: Phys. Rev. E 99.4 (abr. de 2019), pg. 042309. DOI: 10.1103/PhysRevE.99.042309. uRL: https://link.aps.org/doi/10. 1103/PhysRevE.99.042309 (acesso em 28/10/2019) (citado na pg. 53).

[Ozalay et al. 2013] Ozgun Ozalay et al. "The relationship between the anterior corpus callosum size and prefrontal cortex volume in drug-free depressed patients". Em: Journal of Affective Disorders 146.2 (abr. de 2013), pgs. 281-285. Issn: 0165-0327. DOI: $10.1016 /$ j.jad.2012.06.040. URL: http://www.sciencedirect.com/science/ article/pii/S0165032712005071 (acesso em 04/07/2018) (citado na pg. 74).

[Pamplona et al. 2015] Gustavo S. P. Pamplona, Gérson S. Santos Neto, Sara R. E. Rosset, Baxter P. Rogers e Carlos E. G. SAlmon. "Analyzing the association between functional connectivity of the brain and intellectual performance". Em: Front Hum Neurosci 9 (fev. de 2015). ISSN: 1662-5161. DoI: 10.3389/fnhum.2015. 00061. URL: https://www.ncbi.nlm.nih.gov/pmc/articles/PMC4322636/ (acesso em 15/08/2018) (citado na pg. 64).

[PAUl et al. 2007] Lynn K. PAUl et al. "Agenesis of the corpus callosum: genetic, developmental and functional aspects of connectivity”. en. Em: Nature Reviews Neuroscience 8.4 (abr. de 2007), pgs. 287-299. ISSN: 1471-003X. DOI: 10.1038/nrn2107. URL: http://www.nature.com/nrn/journal/v8/n4/full/nrn2107.html (citado na pg. 73).

[Penrose 2003] Mathew Penrose. Random Geometric Graphs. Oxford Studies in Probability 5, mai. de 2003. ISBN: 978-0-19-850626-3 (citado nas pgs. 1, 66).

[Pellegrini et al. 2004] Matteo Pellegrini, David Haynor e Jason M. Johnson. "Protein interaction networks”. eng. Em: Expert Review of Proteomics 1.2 (ago. de 2004), pgs. 239-249. IsSN: 1744-8387. DOI: 10.1586/14789450.1.2.239 (citado na pg. 1).

[Power et al. 2012] Jonathan D. Power, Kelly A. Barnes, Abraham Z. Snyder, Bradley L. Schlaggar e Steven E. Petersen. "Spurious but systematic correlations in functional connectivity MRI networks arise from subject motion”. eng. Em: 
REFERÊNCIAS

NeuroImage 59.3 (fev. de 2012), pgs. 2142-2154. ISSN: 1095-9572. DOI: 10.1016/j. neuroimage.2011.10.018 (citado nas pgs. 64, 75).

[Preciado e Rahimian 2015] Victor M. Preciado e M. Amin Rahimian. "MomentBased Spectral Analysis of Random Graphs with Given Expected Degrees”. Em: arXiv:1512.03489 [physics, stat] (dez. de 2015). arXiv: 1512.03489. uRL: http://arxiv. org/abs/1512.03489 (acesso em 18/01/2018) (citado na pg. 21).

[Qiu e Antonik 2017] Robert C. Qiu e Paul Antonik. Smart Grid using Big Data Analytics: A Random Matrix Theory Approach. en. Google-Books-ID: 6oD5DQAAQBAJ. John Wiley \& Sons, jan. de 2017. ISBN: 978-1-118-71680-9 (citado na pg. 15).

[RAichle, MacLeod et al. 2001] Marcus E. Raichle, Ann Mary MacLeod et al. "A default mode of brain function". en. Em: PNAS 98.2 (jan. de 2001), pgs. 676-682. IsSN: 0027-8424, 1091-6490. DOI: 10.1073/pnas.98.2.676. URL: http://www.pnas.org/ content/98/2/676 (acesso em 18/06/2018) (citado na pg. 61).

[Roland et al. 2017] Jarod L. Roland et al. "On the role of the corpus callosum in interhemispheric functional connectivity in humans". en. Em: PNAS 114.50 (dez. de 2017), pgs. 13278-13283. ISSN: 0027-8424, 1091-6490. DoI: 10.1073/pnas. 1707050114. URL: http:// www. pnas . org / content / 114/50/13278 (acesso em 05/06/2018) (citado nas pgs. 61, 74).

[Rombouts et al. 2009] Serge A. R. B. Rombouts et al. "Model-free group analysis shows altered BOLD FMRI networks in dementia”. eng. Em: Hum Brain Mapp 30.1 (jan. de 2009), pgs. 256-266. ISSN: 1097-0193. DOI: 10.1002/hbm.20505 (citado nas pgs. 3,60$)$.

[Rousseeuw 1987] Peter J. Rousseeuw. "Silhouettes: A graphical aid to the interpretation and validation of cluster analysis". Em: fournal of Computational and Applied Mathematics 20 (nov. de 1987), pgs. 53-65. IssN: 0377-0427. DOI: 10.1016/03770427(87 ) 90125 - 7. URL: http : / www. sciencedirect.com / science / article / pii / 0377042787901257 (citado na pg. 65).

[Raichle e Snyder 2007] Marcus E. Raichle e Abraham Z. Snyder. "A default mode of brain function: a brief history of an evolving idea”. eng. Em: Neuroimage 37.4 (out. de 2007), 1083-1090, discussion 1097-1099. ISSN: 1053-8119. DOI: 10.1016/j. neuroimage.2007.02.041 (citado na pg. 61).

[Rubinov e Sporns 2010] Mikail Rubinov e Olaf Sporns. "Complex network measures of brain connectivity: Uses and interpretations”. Em: NeuroImage. Computational Models of the Brain 52.3 (set. de 2010), pgs. 1059-1069. ISSN: 1053-8119. DOI: 10.1016/j.neuroimage.2009.10.003. URL: http://www.sciencedirect.com/science/ article/pii/S105381190901074X (citado nas pgs. 61, 64).

[SAntos et al. 2016] Suzana de Siqueira Santos, Daniel Yasumasa TAKahashi, João Ricardo Sato, Carlos Eduardo Ferreira e André Fujita. "Statistical methods 
in graphs: parameter estimation, model selection, and test.” Em: Mathematical foundations and applications of graph entropy. Ed. por Yongtang SHI, Sueliang LI e Matthias DeHmer. Wiley-VCH, 2016 (citado nas pgs. 14, 19-22, 57).

[SAto et al. 2015] J. R. Sato, M. Vidal, S. S. Santos, K. B. Massirer e A. Fujita. "Complex network measures in Autism Spectrum Disorders". Em: IEEE/ACM Transactions on Computational Biology and Bioinformatics PP.99 (2015), pgs. 1-1. ISSN: 1545-5963. DoI: 10.1109/TCBB.2015.2476787 (citado na pg. 3).

[Schipul et al. 2012] Sarah E. Schipul, Diane L. Williams, Timothy A. Keller, Nancy J. Minshew e Marcel Adam Just. "Distinctive Neural Processes during Learning in Autism”. en. Em: Cerebral Cortex 22.4 (abr. de 2012), pgs. 937-950. Issn: 1047-3211, 1460-2199. DOI: 10.1093/cercor/bhr162. URL: http://cercor.oxfordjournals.org/ content/22/4/937 (citado na pg. 3).

[Schaer et al. 2013] Marie Schaer et al. "Decreased frontal gyrification correlates with altered connectivity in children with autism". English. Em: Front. Hum. Neurosci. 7 (2013). ISSN: 1662-5161. DOI: 10.3389/fnhum.2013.00750. URL: https://www. frontiersin.org/articles/10.3389/fnhum.2013.00750/full (acesso em 04/07/2018) (citado na pg. 74).

[Scotт 1992] David W. Scotr. Multivariate Density Estimation: Theory, Practice, and Visualization. English. 1st edition. New York: Wiley, ago. de 1992. ISBN: 978-0-47154770-9 (citado nas pgs. 30, 33).

[SEeley et al. 2007] William W. SEeley et al. "Dissociable intrinsic connectivity networks for salience processing and executive control”. eng. Em: F. Neurosci. 27.9 (fev. de 2007), pgs. 2349-2356. ISSN: 1529-2401. DOI: 10.1523/JNEUROSCI.558706.2007 (citado na pg. 60).

[Stephan e Friston 2009] K. E. Stephan e K. J. Friston. "Functional Connectivity". Em: Encyclopedia of Neuroscience. Ed. por Larry R. SQuirE. Oxford: Academic Press, jan. de 2009, pgs. 391-397. ISBN: 978-0-08-045046-9. DOI: 10.1016/B978008045046-9.00308-9. URL: http://www.sciencedirect.com/science/article/pii/ B9780080450469003089 (acesso em 24/08/2019) (citado na pg. 64).

[Shannon 1948] C. E. Shannon. "A Mathematical Theory of Communication”. en. Em: Bell System Technical fournal 27.3 (1948), pgs. 379-423. IssN: 1538-7305. DOI: 10.1002/j.1538-7305.1948.tb01338.x. URL: http://onlinelibrary.wiley.com/doi/10. 1002/j.1538-7305.1948.tb01338.x/abstract (citado na pg. 25).

[Silverman 1986] B. W. Silverman. Density Estimation for Statistics and Data Analysis. English. Boca Raton: Chapman e Hall, jan. de 1986. ISBN: 9780412246203 (citado nas pgs. $30,33,44)$.

[Shi e Malik 2000] Jianbo Shi e Jitendra Malik. "Normalized cuts and image segmentation". Em: IEEE Transactions on pattern analysis and machine intelligence 22.8 (2000), pgs. 888-905 (citado na pg. 65). 
REFERÊNCIAS

[SMith et al. 2011] Stephen M. Smith et al. "Network modelling methods for FMRI". Em: NeuroImage 54.2 (jan. de 2011), pgs. 875-891. ISSN: 1053-8119. DOI: 10.1016/j. neuroimage.2010.08.063. uRL: http://www.sciencedirect.com/science/article/pii/ S1053811910011602 (acesso em 19/08/2019) (citado na pg. 64).

[SNijders e Nowicki 1997] Tom A. B. Snijders e Krzysztof Nowicki. "Estimation and Prediction for Stochastic Blockmodels for Graphs with Latent Block Structure". en. Em: fournal of Classification 14.1 (jan. de 1997), pgs. 75-100. IssN: 0176-4268, 1432-1343. DOI: 10.1007/s003579900004. URL: https://link.springer.com/article/10. 1007/s003579900004 (citado nas pgs. 1, 2, 15, 53).

[Sturges 1926] Herbert A. Sturges. "The Choice of a Class Interval". Em: fournal of The American Statistical Association 21.153 (1926), pgs. 65-66. DOI: 10.1080/ 01621459.1926.10502161 (citado nas pgs. 30, 33).

[TAKahashi et al. 2012] Daniel Yasumasa TAKahashi, João Ricardo Sato, Carlos Eduardo Ferreira e André Fujita. "Discriminating Different Classes of Biological Networks by Analyzing the Graphs Spectra Distribution”. Em: PLoS ONE 7.12 (2012), e49949. DoI: 10.1371/journal.pone.0049949. URL: http://dx.doi.org/10.1371/ journal.pone.0049949 (citado nas pgs. 2-4, 26, 28, 29, 32, 33, 53, 55, 57, 66, 77).

[Tang, Athreya, Sussman, Lyzinski, Park et al. 2017] Minh Tang, Avanti Athreya, Daniel L. Sussman, Vince Lyzinski, Youngser PARK et al. "A Semiparametric Two-Sample Hypothesis Testing Problem for Random Graphs”. Em: fournal of Computational and Graphical Statistics 26.2 (abr. de 2017), pgs. 344-354. ISSN: 1061-8600. DOI: 10.1080/10618600.2016.1193505. URL: http://dx.doi.org/10.1080/ 10618600.2016.1193505 (citado na pg. 2).

[Tang, Athreya, Sussman, Lyzinski e Priebe 2017] Minh Tang, Avanti Athreya, Daniel L. Sussman, Vince Lyzinski e Carey E. Priebe. "A nonparametric two-sample hypothesis testing problem for random graphs”. EN. Em: Bernoulli 23.3 (ago. de 2017), pgs. 1599-1630. ISSN: 1350-7265. DOI: 10.3150/15-BEJ789. URL: https:// projecteuclid.org/euclid.bj/1489737619 (citado na pg. 2).

[ToAl et al. 2010] F. ToAl et al. "Clinical and anatomical heterogeneity in autistic spectrum disorder: a structural MRI study”. eng. Em: Psychological Medicine 40.7 (jul. de 2010), pgs. 1171-1181. ISSN: 1469-8978. DOI: 10.1017/S0033291709991541 (citado nas pgs. 59, 60).

[Travers et al. 2015] Brittany G. Travers et al. "Atypical development of white matter microstructure of the corpus callosum in males with autism: a longitudinal investigation”. en. Em: Molecular Autism 6.1 (mar. de 2015), pg. 15. Issn: 2040-2392. DOI: 10.1186/s13229-015-0001-8. URL: http://www.molecularautism.com/content/6/1/ 15/abstract (citado nas pgs. 3, 61, 73).

[TsAi 2013] Luke Y. TsaI. “Asperger's Disorder will be Back”. en. Em: fournal of Autism and Developmental Disorders 43.12 (mai. de 2013), pgs. 2914-2942. ISsN: 0162-3257, 
1573-3432. DOI: 10.1007/s10803-013-1839-2. URL: http://link.springer.com/article/ 10.1007/s10803-013-1839-2 (citado na pg. 59).

[Tran et al. 2013] Linh V. Tran, Van H. Vu e Ke Wang. "Sparse Random Graphs: Eigenvalues and Eigenvectors”. Em: Random Struct. Algorithms 42.1 (jan. de 2013), pgs. 110-134. ISSN: 1042-9832. DOI: 10.1002/rsa.20406. URL: http://dx.doi.org/10. 1002/rsa.20406 (citado nas pgs. 2, 14, 18).

[Tzourio-Mazoyer et al. 2002] N. Tzourio-Mazoyer et al. "Automated anatomical labeling of activations in SPM using a macroscopic anatomical parcellation of the MNI MRI single-subject brain”. eng. Em: Neuroimage 15.1 (jan. de 2002), pgs. 273289. ISSN: 1053-8119. DOI: 10.1006/nimg.2001.0978 (citado na pg. 63).

[Van Mieghem 2010] Piet Van Mieghem. Graph Spectra for Complex Networks. Cambridge: Cambridge University Press, 2010. ISBN: 9780511921681 . URL: http://ebooks. cambridge.org/ebook.jsf?bid=CBO9780511921681 (citado nas pgs. 2, 65).

[C. N. Vidal et al. 2006] Christine N. Vidal et al. "Mapping corpus callosum deficits in autism: an index of aberrant cortical connectivity”. eng. Em: Biological Psychiatry 60.3 (ago. de 2006), pgs. 218-225. ISSN: 0006-3223. Dor: 10.1016/j.biopsych.2005.11. 011 (citado nas pgs. 61, 73).

[Venables e Ripley 2003] W. N. Venables e B. D. Ripley. Modern Applied Statistics with S. English. 4th edition. New York: Springer, set. de 2003. ISBN: 978-0-38795457-8 (citado nas pgs. 30, 33).

[WANG et al. 2010] H. WANG et al. "Effect of tumor resection on the characteristics of functional brain networks". Em: Phys. Rev. E 82.2 (ago. de 2010), pg. 021924. Dor: 10.1103/PhysRevE.82.021924. uRL: https://link.aps.org/doi/10.1103/PhysRevE.82. 021924 (acesso em 22/08/2019) (citado na pg. 61).

[Weissenbacher et al. 2009] Andreas Weissenbacher et al. "Correlations and anticorrelations in resting-state functional connectivity MRI: a quantitative comparison of preprocessing strategies”. eng. Em: Neuroimage 47.4 (out. de 2009), pgs. 1408-1416. ISSN: 1095-9572. DOI: 10.1016/j.neuroimage.2009.05.005 (citado na pg. 64).

[Weng et al. 2010] Shih-Jen Weng et al. "Alterations of resting state functional connectivity in the default network in adolescents with autism spectrum disorders". eng. Em: Brain Research 1313 (fev. de 2010), pgs. 202-214. ISSN: 1872-6240. DOI: 10.1016/j.brainres.2009.11.057 (citado nas pgs. 60, 73).

[Whitfield-Gabrieli et al. 2009] Susan Whitfield-Gabrieli et al. "Hyperactivity and hyperconnectivity of the default network in schizophrenia and in first-degree relatives of persons with schizophrenia”. eng. Em: Proc. Natl. Acad. Sci. U.S.A. 106.4 (jan. de 2009), pgs. 1279-1284. ISSN: 1091-6490. DOI: 10.1073/pnas.0809141106 (citado nas pgs. 3, 60, 67). 
REFERÊNCIAS

[Wigner 1958] Eugene P. Wigner. "On the Distribution of the Roots of Certain Symmetric Matrices”. Em: Annals of Mathematics 67.2 (1958), pgs. 325-327. IsSN: 0003486X. DOI: 10.2307/1970008. URL: http://www.jstor.org/stable/1970008 (citado na pg. 13).

[WolfF et al. 2012] Jason J. WolfF et al. "Differences in white matter fiber tract development present from 6 to 24 months in infants with autism”. eng. Em: Am $\mathcal{f}$ Psychiatry 169.6 (jun. de 2012), pgs. 589-600. ISSN: 1535-7228. Dor: 10.1176/appi. ajp.2011.11091447 (citado na pg. 59).

[Watts e Strogatz 1998] Duncan J. Watts e Steven H. Strogatz. "Collective dynamics of 'small-world' networks”. en. Em: Nature 393.6684 (jun. de 1998), pgs. 440442. ISSN: 0028-0836. DOI: 10.1038/30918. uRL: http://www.nature.com/nature/ journal/v393/n6684/abs/393440a0.html (citado nas pgs. 1, 20, 66).

[Zhao et al. 2018] Feng Zhao, Han Zhang, Islem Rekik, Zhiyong An e Dinggang Shen. "Diagnosis of Autism Spectrum Disorders Using Multi-Level High-Order Functional Networks Derived From Resting-State Functional MRI”. English. Em: Front. Hum. Neurosci. 12 (2018). ISSN: 1662-5161. DOI: 10.3389/fnhum.2018.00184. URL: https://www.frontiersin.org/articles/10.3389/fnhum.2018.00184/full (acesso em 19/05/2018) (citado na pg. 59).

[Zhou et al. 2007] Yuan Zhou et al. "Functional disintegration in paranoid schizophrenia using resting-state fMRI". eng. Em: Schizophr. Res. 97.1-3 (dez. de 2007), pgs. 194-205. ISSN: 0920-9964. DOI: 10.1016/j.schres.2007.05.029 (citado nas pgs. 3, $61)$.

[Zwaigenbaum et al. 2015] Lonnie Zwaigenbaum et al. "Early Identification of Autism Spectrum Disorder: Recommendations for Practice and Research”. eng. Em: Pediatrics 136 Suppl 1 (out. de 2015), S10-40. ISSN: 1098-4275. DOI: 10.1542/peds.20143667C (citado na pg. 59). 
